$$
\begin{gathered}
\text { ESTIMAÇÃO DE MODELOS } \\
\text { COM TENDÊNCIAS COMUNS } \\
\text { PARA PROCESSOS ESTOCÁSTICOS } \\
\text { MULTIVARIADOS COM CO-INTEGRAÇÃO : } \\
\text { UMA APLICAÇÃO À PARIDADE DE PODER } \\
\text { DE COMPRA FRANCO FRANCÊS-DÓLAR }
\end{gathered}
$$

\title{
Maria Laura Hartpence
}

\author{
DISSERTAÇÃO APRESENTADA \\ AO \\ INSTITUTO DE MATEMÁTICA E ESTATÍSTICA \\ DA UNIVERSIDADE DE SÃO PAULO \\ PARA OBTENÇÃO DE GRAU DE MESTRE \\ EM \\ ESTATÍSTICA \\ Área de Concentração : Estatística \\ Orientador : Prof. Dr. Pedro Luiz Valls Pereira \\ - São Paulo, Julho de 1994 -
}


A

Marc

e

a meus filhos

Alexandre, Caroline e Paola 


\section{Agradecimentos}

Quero agradecer a todos que contribuíram para a realização deste trabalho, em particular :

- ao Prof. Pedro Valls Pereira, meu orientador, pelas muitas horas a que ele se dedicou para me esclarecer e transmitir seus conhecimentos;

- aos Profs. Pedro Morettin e Fernando de Holanda Barbosa pelos seus valiosos comentários e sugerências com relação a este trabalho;

- a todos os professores do IME, pela sua enorme contribuição à minha formação na área da Estatística;

- a Maria Angélica Longo e Monalisa Guarda, pela sua ajuda na edição deste trabalho em português;

- a meu marido Marc, pela sua paciência e incentivo nestes anos de estudo. 


\section{Resumo}

Este trabalho apresenta um procedimento (desenvolvido por King et alli., 1991 e Melander et alli., 1992) que permite a estimação da representação de Tendências Comuns de um processo estocástico multivariado, com vetores co-integrados. A função de Respostas de Impulso neste contexto é também obtida, o que permitirá investigar como o sistema é afetado por inovações nas tendências.

Um processo estocástico com co-integração pode admitir as seguintes representações : 1) Modelo Autoregressivo Vetorial (VAR); 2) Mecanismo de Correção de Erro (MCE); 3) VAR Restrito; 4) Wold, e finalmente, 5) Tendências Comuns.

Será mostrado como a partir da estimação da representação do MCE, os parâmetros da representação VAR e VAR Restrito, podem ser obtidos. O conhecimento dos parâmetros do VAR restrito permitem o cálculo dos parâmetros da representação de Wold. Finalmente os parâmetros da representação de Tendências Comuns são identificados usando : 1) as relações que existem entre os parâmetros das representações de Wold e de Tendências, 2) as restrições impostas pela presença de cointegração e 3) restrições adicionais na matriz de variânciacovariância do processo, e em alguns casos, outras restrições, que devemos impor para que o sistema seja exatamente identificado.

Usando as técnicas descritas, é feita uma aplicação para um vetor de dimensão 5 compreendendo as seguintes variáveis : preços e taxas de juros na França e nos EUA, e a taxa de câmbio FFr/US\$. 


\section{Summary}

This work describes a technique (developed by king et alli., 1991 and Melander et alli., 1992) which allows the estimation of the Common Trend representation for a multivariate stochastic process, with cointegration. Impulse Response Functions are also calculated in this context, so that the response of the system to innovations to the trends can be investigated.

A process of this kind can have the following representations : 1) Vector Auto-regressive (VAR); 2) Error Correction Model (ECM); 3) Restricted VAR; 4) Wold representation and finally, 5) Common Trend representation.

It will be shown how the VAR and the Restricted VAR parameters can be obtained taking the ECM estimated parameters as a point of departure. Parameters from the Restricted VAR representation will allow for the obtention of the Wold representation parameters. Finally, the parameters of the Common Trend representation are identified using : 1) the relationships between the Wold and the Common Trend representation, 2) the restrictions imposed by co-integration and 3) additional restrictions in the covariance matrix of the process, and, in some cases, other restrictions which have to be imposed for the system to be exactly identified.

An application of the techniques described above is done on a 5-dimensional vector with the following variables : prices and interest rates in France and the USA, and the exchange rate FF./US\$. 


\section{Índice}

Capítulo 1 - Introdução ..................... 1

1.1 Modelando Flutuações Macroeconômicas

através da Decomposição de Séries em

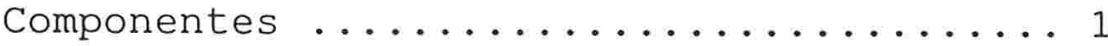

1.1.1 Modelos de Tendência Estacionária

(TE) versus Modelos de Diferença

Estacionária (DE) ............... 2

1.1.2 Representação Estocástica do

Componente Permanente............4

1.2 A Estimação de Modelos de Componente

Permanente Estocástico com

Tendências Comuns .............. 5

Capítulo 2 - As Representações de Modelo Autoregressivo Vetorial (VAR) e de Mecanismo de Correção de Erro (MCE) ................... 9

2.1 A Representação VAR - Modelo Autoregressivo Vetorial................ 9

2.1.1 Características Gerais ....... 9

2.1.2 Modelos VAR (p) Estacionários ...10

2.1.3 Estimação de Modelos $\operatorname{VAR}(p)$

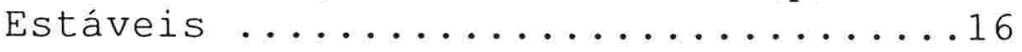

2.1.3.1 Estimação de Mínimos

Quadrados Multivariada .......16

2.1.3.2 Outras Técnicas de

Estimação ...............18

2.1.4 Modelos VARMA e Modelos MA de

Ordem Finita .................18

2.2 A Representação MCE - Mecanismo de

Correção de Erro ..................20

2.2.1 Integração e Co-integração ....20

2.2.2 A Representação MCE - Mecanismo

de Correção de Erro .............22

2.2.3 Estimação de Modelos MCE......25

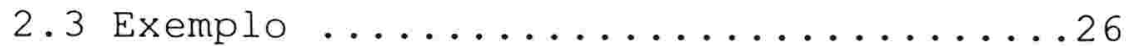


Capítulo 3 - A Representação VAR "Restrita"..........29

3.1 A Representação VAR "Restrita".......29

3.2 Calculando a Representação VAR a

partir da Representação VAR Restrita ....32

3.3 Exemplo .........................

Capítulo 4 - As Representações de Wold e de

Tendências Comuns .................

4.1 A Representação de Wold ...........36

4.2 A Representação de Tendências Comuns .38

4.3 Exemplo ......................

Capítulo 5 - Identificação dos parâmetros da

Representação de Tendências Comuns .....43

5.1 Procedimento de Identificação ......43

5.2 Exemplo .................46

Capítulo 6 - Cálculo da Função Resposta de Impulso

Associada à Representação de Tendências

Comuns ..................... 51

6.1 Procedimento de Identificação ......51

6.2 Exemplo ....................53

Capítulo 7 - Uma Aplicação da Metodologia a Séries

Macroeconômicas da França e dos EUA .....55

7.1 Uma Descrição das Teorias da

Paridade do Poder de Compra e da Paridade

de Taxas de Juros não Coberta........55

7.2 Resultados empíricos ...........58

7.2.1 Propriedades de Integração e

Co-integração dos Dados ..........58

7.2.2 Estimação da Representação MCE..62

7.2.3 Análise do Espaço de

Co-integração ..............6. 65

7.2.4 Alguns Testes sobre Restrições

do Espaço de Co-integração .......70

7.2.5 A Representação VAR Restrita ...73

7.2 .6 A Representação de Wold ......75

7.2.7 Identificação dos Parâmetros da

Representação de Tendências Comuns ..76

7.2.8 Cálculo de Respostas de Impulsos

Associados a Choques nos Componentes

Permanente e Transitório .........82 
Anexo Capítulo 7 - Funções de

Autocorrelação e Autocorrelação Parcial

das $1^{\text {as }}$ Diferenças das Séries do Modelo

e das Relações de Co-integração.....889

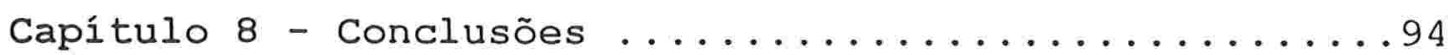

Apêndice A - Algums Resultados de Algebra Matricial . .997

Apêndice B - Somas Infinitas de Matrices e

Convergência Estocástica ...........104

Apêndice C - Cálculo dos Coeficientes do Polinomio na Representação de MCE .....108

Apêndice D - O Método de Johansen para estimar Processos AR com Co-integração .......110

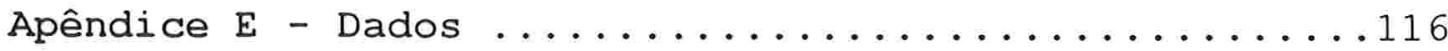

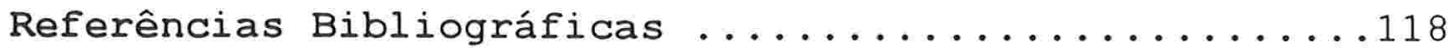




\section{Capítulo 1}

\section{Introdução}

o objetivo deste trabalho será a apresentação, no contexto multivariado, de um método de estimação de modelos de componentes permanente e transitório, onde ambos são estocásticos. A hipótese de trabalho será a presença de cointegração entre as séries que constituem 0 vetor estocástico em estudo, o que dá lugar ao chamados modelos de Tendências Comuns. Para a estimação destes modelos, partiremos da estimação de parâmetros em outros tipos de representações do processo estocástico.

Nesta introdução, explicaremos, na seção 1.1, o porquê da escolha de modelos de componente permanente estocástico em vez de usar modelos de componente permanente determinístico.

$\mathrm{Na}$ seção 1.2 serão definidas as principais características do modelo de Componente Permanente Estocástico com Tendências Comuns. Apresentaremos também o enfoque deste trabalho e a organização do mesmo.

\subsection{Modelando Flutuações Macroeconômicas através da Decomposição de Séries em Componentes}

A decomposição de uma série temporal em dois componentes: um componente secular, denominado também de crescimento ou permanente, e um componente cíclico ou transitório, têm constituído nos últimos anos uma das práticas mais usadas no tratamento de séries econômicas tais como produção, inflação, taxas de juros, investimentos, etc. entre outras.

O componente secular ou permanente é geralmente suposto ser não estacionário, no sentido de que o valor esperado do mesmo e/ou de sua variância e/ou função de autocovariância variam com o tempo. O componente transitório ou cíclico é assumido ser estacionário, i.e. média e variância são independentes do tempo, e as autocovariâncias dependem dos intervalos entre os tempos. 


\subsubsection{Modelos de Tendência Estacionária (TE) versus Modelos de Diferença Estacionária (DE) ${ }^{1}$}

Até inícios dos anos 80, a técnica comumente usada, dentro da prática de decomposição de uma série em componentes permanente e transitório, era o ajuste da série por uma tendência polinomial deterministica, representando o componente secular ou permanente. Os resíduos eram interpretados como os ciclos ou componente transitório. Uma das versões mais populares dentro desta última técnica é a adoção de uma tendência linear determinística, ou seja, modelos, no caso univariado, do tipo :

$$
\begin{aligned}
& z_{t}=\alpha+\beta t+c_{t} \\
& \phi(L) c_{t}=\theta(L) u_{t} ; u_{t} \approx i \text { i. } d\left(0, \sigma_{u}^{2}\right)
\end{aligned}
$$

onde $z_{t}$ é o logaritmo natural da variável em estudo, $\alpha$ e $\beta$ são parâmetros fixos, L é o operador desfasagem e $\phi(L)$ e $\theta(L)$ são polinômios em $L$ que satisfazem as condições de estacionariedade e invertibilidade, implicando que $c_{t}$ é um processo estacionário.

O processo estocástico (1.1) é conhecido na literatura por Processo de Tendência Estacionária (TE). Note que (1.1) é um processo não estacionário, sendo que a média do processo varia com o tempo. O determinismo deste tipo de processo é capturado no fato de que embora a autocorrelação de $c_{t}$ possa ser usada para projeções a curto prazo, as projeções a longo prazo estão dadas fundamentalmente pela média do processo $(\alpha+\beta t)$-componente permanente-, de modo que eventos passados não alteram expectativas a longo prazo.

Uma classe alternativa de processo estocástico não estacionário é a classe em que as diferenças de primeira ou maior ordem constituem processos ARMA estacionários e invertíveis. Estes processos são denominados, às vezes, na literatura, de Processos de Diferença Estacionária (DE). Um exemplo típico deste tipo de processos é o DE de primeira ordem, expresso em logaritmos naturais :

$$
\begin{aligned}
& (1-L) z_{t}=\beta+d_{t} \\
& \delta(L) d_{t}=\lambda(L) u_{t} u_{t} \approx i \cdot i \cdot d\left(0, \sigma_{u}^{2}\right)
\end{aligned}
$$

\footnotetext{
${ }^{1}$ Seguindo Nelson \& Plosser (1982)
} 
onde $\delta(L)$ e $\lambda(L)$ são polinômios satisfazendo condições de estacionariedade e invertibilidade. Substituindo recursivamente em (1.2) permite escrever :

$$
z_{t}=z_{0}+\beta t+\sum_{j=1}^{t} d_{t}
$$

As equações (1.1) e (1.3) indicam que os dois tipos de processos podem ser escritos como uma função linear do tempo mais um desvio desta função. Entretanto, o intercepto em (1.1) é um parâmetro fixo, enquanto que em (1.3) depende de eventos históricos. Por outro lado, os desvios da tendência em (1.1) constituem um processo estacionário, sendo que os mesmos em (1.3) conformam uma acumulação de erros que é não estacionária.

A diferença fundamental entre os dois processos pode ser observada também em termos das raízes dos polinômios Autoregressivos (AR) e de Média Móvel (MA). No modelo TE, a primeira diferença dá :

$$
\phi(\mathrm{L})\left[(1-\mathrm{L}) \mathrm{z}_{\mathrm{t}}\right]=\beta \phi(1)+(1-\mathrm{L}) \theta(\mathrm{L}) \mathrm{u}_{\mathrm{t}}
$$
em 1.

onde $\phi(1)$ é a constante obtida avaliando o polinômio $\phi(L)$

Para o modelo $\mathrm{DE}$, obtem-se :

$$
\delta(\mathrm{L})(1-\mathrm{L}) z_{\mathrm{t}}=\beta \delta(\mathrm{l})+\lambda(\mathrm{L}) \mathrm{u}_{\mathrm{t}}
$$

A expressão (1.4) indica a presença de uma raíz unitária no polinômio MA do processo ARMA descrevendo a série $\left[(1-L) z_{t}\right] ;$ a expressão (1.5), por outro lado, envolve uma raíz unitária no polinômio $A R$, do processo ARMA para $z_{t}$.

A discussão precedente possibilita afirmar, em teoria, que se uma série é gerada por um processo TE, não poderemos rejeitar a hipótese nula de uma raíz unitária na parte MA da representação ARMA da série $\left[(1-\mathrm{L}) \mathrm{z}_{\mathrm{t}}\right]$. Do mesmo modo, se a série for gerada por um processo DE da primeira ordem, não poderemos rejeitar a hipótese nula de uma raíz unitária na parte AR, da representação ARMA de $z_{t}$. 


\subsubsection{Representação Estocástica do Componente Permanente}

Nelson e Plosser (1982), analizaram um grande número de séries econômicas para os EUA (médias anuais, começando em 1860-1909 até 1970), realizando testes que, usando alguns elementos teóricos mencionados na seção anterior, apresentam evidência consistente com a representação DE para séries não estacionárias. ${ }^{2}$

Em outras palavras, a análise de Nelson e Plosser sugere que as séries de tempo econômicas não contém tendências determinísticas, mas tendências estocásticas, i.e. são processos da classe DE.

Prosseguindo com a idéia de que uma série de tempo econômica pode ser decomposta em dois componentes : um permanente, não estacionário e outro transitório, este último estacionário, e assumindo que a série pertence à classe $\mathrm{DE}$, então $O$ componente permanente deve também pertencer à classe DE. Esta decomposição da série pode ser expressa como :

$$
\begin{aligned}
y_{t} & =\bar{y}_{t}+c_{t} \\
& =(1-I)^{-1} \theta(L) u_{t}+\psi(L) u_{t}
\end{aligned}
$$

onde :

$$
\begin{aligned}
& \bar{Y}_{t}=\left(1-L^{-1} \theta(L) u_{t} \text { or } \quad(1-L) \bar{Y}_{t}=\theta(L) u_{t}\right. \\
& c_{t}=\psi(L) u_{t}
\end{aligned}
$$

$\bar{y}_{t}$ e $C_{t}$ são os componentes permanente e transitório respectivamente. Os polinômios $\theta(L)$ e $\psi(L)$ satisfazem as condições de estacionariedade e invertibilidade, $v_{t} e u_{t}$ são variáveis aleatórias de média 0 , variância constante, e sem autocorrelação serial. A equação (1.6) atribui não estacionariedade ao componente secular ou permanente através

\footnotetext{
${ }^{2}$ As autocorrelações amostrais das séries em níveis começam típicamente com valores en torno de 0,96, decaindo lentamente. As primeiras diferenças apresentam autocorrelações positivas no primeiro lag, para logo cair rapidamente, característica de processos MA de primeira ordem. $\mathrm{Na}$ maior parte das séries, as primeiras diferenças não mostraram evidência de apresentar raízes unitárias nos termos MA. Mais formalmente, Nelson \& Plosser realizaram os testes desenvolvidos por Dickey \& Fuller para testar a hipótese nula DE contra a hipótese alternativa TE. Na maioria dos casos, a hipótese nula não foi rejeitada.
} 
de $(1-L)^{-1}$ e fica claro que ele é estocástico, e não determinístico como em (1.1).

\subsection{A Estimação de Modelos de Componente Permanente Estocástico com Tendências Comuns}

A motivação do presente trabalho é a descrição de técnicas para estimar modelos do tipo da expressão (1.6) na versão multivariada, mais precisamente no contexto de presença de "co-integração" e "tendências comuns", idéias que serão discutidas brevemente a seguir.

Baseado em Melander (1992), são resumidas a seguir as hipóteses de trabalho:

1) As séries temporais podem ser decompostas em componentes permanente (secular) e transitório.

2) O componente permanente, representado por uma tendência, será tratado como um processo estocástico, e não determinístico.

3) Assume-se de que o número de tendências é relativamente baixo quando comparado ao número de variáveis macroeconômicas do vetor em estudo.

Formalizando, podemos formular o mecanismo estatístico gerador (MEG) das séries em estudo como :

$$
x_{t}=x_{0}+\mathrm{K} \tau_{t}+\Phi(\mathrm{L}) v_{t}
$$

onde $\chi_{t}$ é um vetor $n$-dimensional de séries, $\boldsymbol{\chi}_{\mathbf{0}}$ é o vetor constante contendo os valores iniciais de $\chi$. Os componentes não estacionários (permanentes) e estacionários (transitórios) de $\chi_{t}$ estão capturados por $K \tau_{t}$ e $\Phi(L) v_{t}$ respectivamente. $\mathbf{K}$ é uma matriz de dimensão $n \times k$, onde $0<\mathrm{k}<\mathrm{n}$ é o número de tendências do processo. $\tau_{t}$ é a variável de tendência, vetor de dimensão $k \times 1$. A sequência $n$-dimensional $v_{t}$ é um ruido branco com $E\left(v_{t}\right)=0$ e $E\left(v_{t} v^{\prime}{ }_{t}\right)=I_{n}$ matriz identidade de ordem $n$. Assumimos que $v_{\mathbf{s}}=0$ para todo $s \leq 0$. Para que $\Phi(L) v_{t}$ seja estacionário, o polinômio :

$$
\Phi(\mathrm{L})=\sum_{i=0}^{\infty} \Phi_{i} \mathrm{~L}^{\mathrm{i}}
$$


deve ser absolutamente somável (no capítulo 2 explicaremos com mais detalhe o conceito de estacionariedade).

Se a tendência é linearmente determinística, então $\tau_{t}=\mu t$, ou seja, $\tau_{t}-\tau_{t-1}=\mu$, onde $\mu$ é um vetor $k$-dimensional de constantes, com $0<\mathrm{k}<\mathrm{n}$. A idéia de tendência linear estocástica pode ser operacionalizada como um vetor de passeio aleatório com desvío do tipo :

$$
\tau_{t}=\mu+\tau_{t-1}+\varphi_{t}
$$

onde $\left\{\varphi_{t}\right\}$ é um ruido branco $\operatorname{com} E\left(\varphi_{t}\right)=0$ e $E\left(\varphi_{t} \varphi_{t}^{\prime}\right)=I_{k}$. Se $\mathrm{K} \neq 0$, então, $\varphi_{t}$ é um vetor $k$-dimensional de choques estruturais (independentes), com efeitos permanentes em $\chi_{t}$. Deve ser enfatizado que estes choques permanentes podem dar lugar a flutuações transitórias em torno da tendência, isto é, $\boldsymbol{v}_{\mathrm{t}}$ pode conter elementos de $\boldsymbol{\varphi}_{\mathrm{t}}$.

Devemos notar que a hipótese de tendências estocásticas pode ser rejeitada se permitirmos translações em tendências determinísticas para tempos turbulentos. Para permitir translações, tanto em tendências estocásticas como em tendências determinísticas, podemos reformular o modelo em (1.8) e (1.9) da seguinte forma :

$$
\chi_{t}=\chi_{0}+K \tau_{t}+\Phi(L)\left(v_{t}+\Psi D_{t}\right)
$$

e

$$
\tau_{t}=\mu+\mu^{\star} D_{t}+\tau_{t-1}+\varphi_{t}
$$

Aqui $D_{t}$ é um vetor d-dimensional de 0-1 variáveis dummy.

Até aqui assumimos que a dimensão de $\mathrm{K}$ é menor que $\mathrm{n}$, o que parece uma hipótese razoável, e equivale a dizer que existe um número de tendências comuns. Stock e Watson (1988) demonstram que isto resulta em $n-k$ combinações lineares de elementos de $x_{t}$ que são estacionários, mesmo que os elementos tomados independentes sejam não estacionários. Dizemos neste caso que existem $n-k$ vetores co-integrados. A demonstração mais detalhada deste fatos será apresentada no capítulo 4.

Modelos tais como (1.10) e (1.11) não são estimados com facilidade, sendo que eles são formulados em função de variáveis não observadas. o objetivo deste trabalho é 
justamente apresentar um método que permita a estimação dos parâmetros em $(1.10)$ e (1.11), não diretamente mas mediante a estimação dos parâmetros de representações alternativas do processo estocástico $x_{t}$ e logo calculando os parâmetros do modelo (1.10)-(1.11) mediante a identificação das relações existentes entre estes parâmetros e os parâmetros das representações alternativas.

Temos cinco possiveis formas de representar um processo estocástico multivariado não estacionário, com cointegração : 1) Modelo Autoregressivo Vetorial (VAR); 2) Mecanismo de Correção de Erro (MCE); 3) VAR Restrito; 4) Wold, e finalmente, 5) Tendências Comuns.

Essencialmente, o método proposto para estimar o modelo (1.10)-(1.11), consiste em estimar os parâmetros no modelo MCE e partir destas estimativas para obter sequencialmente os parâmetros do modelo VAR restrito, da representação de Wold e, finalmente, da representação de Tendências Comuns.

No capítulo 2, abordamos os conceitos de modelo Autoregressivo Vetorial (VAR), Médias Móveis (MA) e Autoregressivo-Médias Móveis (ARMA) Vetoriais, para logo introduzir $\circ$ conceito de co-integração e de modelo de Mecanismo de Correção de Erro. São apresentados também os métodos de estimação para estas representações.

No capítulo 3 apresentamos a representação VAR restrita e - procedimento de identificação dos parâmetros da representação VAR a partir dos parâmetros da representação VAR restrita.

- capítulo 4 trata da representação de Wold e como, a partir desta, podemos obter a representação de Tendências Comuns.

O capítulo 5 explica detalhadamente o processo de cálculo dos parâmetros do modelo de Tendências Comuns a partir dos parâmetros que já dispomos em representações anteriores.

O capítulo 6 mostra como calcular as funções de Resposta de Impulsos para aquela representação.

Finalmente, o capítulo 7 desenvolve um exemplo de aplicação da metodologia descrita nos capítulos anteriores para um vetor compreendendo as seguintes variáveis : preços e taxas de juros na França e nos EUA, e a taxa de câmbio FFr/US\$. 
Adicionalmente, são apresentados 5 apêndices com alguns resultados de algebra matricial e probabilidade frequentemente usados neste trabalho (apêndices $A$ e B), $O$ cálculo do polinômio em L na representação do MCE (apêndice C), a metodologia de Johansen \& Juselius para a estimação de modelos de MCE (apêndice D) e os dados usados no exemplo desenvolvido no capítulo 7 (apêndice E). 


\section{Capítulo 2}

\section{As Representações de Modelo Autoregressivo Vetorial (VAR) e de Mecanismo de Correção de Erro (MCE)}

Neste capitulo abordamos, rapidamente, as principais
caracteristicas do Modelo Autoregressivo Vetorial (VAR) de
ordem finita, que constitui a primeira representação, para
uma série temporal, mencionada no capitulo 1. São
apresentadas as propriedades dos processos VAR estacionários
e o método de estimação destes modelos. Adicionalmente,
agregam-se as definições, em um contexto mais geral, dos
modelos Autoregressivos-Médias Móveis (VARMA) e Médias
Móveis (MA) vetoriais. Estes temas são tratados na seção
2.1.

A representação do Mecanismo de Correção de Erro (MCE) é tratada logo após, na seção 2.2, introduzindo o conceito de co-integração, assim como o método de estimação daquela representação.

Por último, na seção 2.3, exibimos um exemplo para esclarecer os conceitos discutidos.

\subsection{A Representação VAR - Modelo Autoregressivo Vetorial}

\subsubsection{Características Gerais}

Definição 2.10 modelo ou representação da forma :

$$
\chi_{t}=\rho+A_{1} \chi_{t-1}+A_{2} x_{t-2}+\ldots++A_{p} \chi_{t-p}+\varepsilon_{t}
$$

onde $n \times 1$,

(i) $\chi_{t}=\left(\chi_{1 t}, \ldots, \chi_{n t}\right)^{\prime}$ é um vetor estocástico de dimensão

(ii) as $A_{i}$ são as matrizes dos coeficientes de dimensão $\mathrm{n} \times \mathrm{n}$, 
(iii) $\rho=\left(\rho_{1}, \ldots, \rho_{n}\right)^{\prime}$ é um vetor fixo $n \times 1$ contendo os termos de intercepto, permitindo que a média de $x_{t}$ seja diferente de 0 ,

(iv) $\varepsilon_{\mathrm{t}}=\left(\varepsilon_{1 \mathrm{t}}, \ldots, \varepsilon_{\mathrm{nt}}\right)^{\prime}$ é um ruido branco ou seja, $\mathrm{E}\left(\varepsilon_{\mathrm{t}}\right)=0$, $E\left(\varepsilon_{t} \varepsilon_{t}^{\prime}\right)=\Sigma$ é uma matriz não singular e $E\left(\varepsilon_{t} \varepsilon_{s}^{\prime}\right)=0$ para $s \neq t$. Alternativamente $\left\{\varepsilon_{\mathrm{t}}\right\}$ pode ser um processo de inovação n-dimensional, ou seja, com $E\left(\varepsilon_{t} / E_{t-1}\right)=0$ e $E\left(\varepsilon_{t} \varepsilon_{t}^{\prime}\right)=\Sigma$.

é denominado por modelo $\operatorname{VAR}(\mathrm{p})$ (Modelo Autoregressivo Vetorial de ordem $\mathrm{p}$ ).

A expressão (2.1) pode ser escrita igualmente como :

$$
\mathrm{A}(\mathrm{L}) \chi_{\mathrm{t}}=\rho+\varepsilon_{\mathrm{t}}
$$

onde A(L) é :

$$
\mathrm{A}(\mathrm{L})=\mathrm{I}_{\mathrm{n}}-\mathrm{A}_{1} \mathrm{~L}-\ldots-\mathrm{A}_{\mathrm{p}} \mathrm{I}^{\mathrm{P}}
$$

\subsubsection{Modelos VAR(p) Estacionários}

Definição 2.2 Seja $\left\{\chi_{t}\right\}$ um processo estocástico de dimensão $n$. O processo $\chi_{t}$ é fracamente estacionário ou estacionário de segunda ordem se :

(i) $E\left(\boldsymbol{\chi}_{t}\right)=\mu<\infty$ para todo $t$,

(ii) $E\left[\left(\chi_{t}-\mu\right)\left(\chi_{t}-\mu\right)^{\prime}\right]=\Sigma_{\chi}<\infty$ para todo $t$,

(iii) $E\left[\left(\chi_{t}-\mu\right)\left(\chi_{t-h}-\mu\right)^{\prime}\right]=\Gamma_{\chi}(h)<\infty$ para todo $t e h$,

onde $\boldsymbol{\mu}$ é um vetor $\mathrm{n}$-dimensional das médias, $\Sigma_{\chi}$ é a matriz de covariância de dimensão $n \times n$ e $\Gamma_{\chi}(h)$ é a matriz de autocovariância de dimensão $n \times n, \operatorname{com} \Gamma_{\chi}(0)=\Sigma_{\chi}$.

No futuro, quando falarmos de processos estacionários, estaremos nos referindo a modelos fracamente estacionários, também chamados de estacionários em sentido restrito.

Para estudar as condições de estacionariedade na representação $\operatorname{VAR}(\mathrm{p})$, defina o operador : 


$$
\begin{aligned}
& \Phi(L):=\sum_{i=0}^{\infty} \Phi_{i} L^{i} \text { tal que } \\
& \Phi(L) A(L)=I_{n}
\end{aligned}
$$

O operador $\Phi(L)$, se existir, é o inverso de $A(L)$, e é denotado às vezes por $A(L)^{-1}$. Se $\Phi(L)$ existe, o operador $A(L)$ é chamado de invertível.

Definição 2.3 Seja o processo VAR(p) definido em (2.1) um processo estacionário. Este processo é chamado de causal (ou mais específicamente ser uma função causal de $\boldsymbol{\varepsilon}_{\boldsymbol{t}}$ ) se existe uma sequência de constantes $\left\{\Phi_{i}\right\}$ tal que :

$$
\sum_{i=0}^{\infty}\left|\Phi_{i}\right|<\infty \quad \quad \quad \chi_{t}=\sum_{i=0}^{\infty} \Phi_{i} \varepsilon_{t-i}
$$

Proposição 2.1 Seja $A(L)$ uma matriz polinomial. Se $|A(z)| \neq 0$ para $|z| \leq 1$, (ou seja, as raízes de $|A(z)|$ estão todas fora do círculo unitário), então $A(L)^{-1}=\Phi(L)$ onde :

$$
\Phi(L):=\sum_{i=0}^{\infty} \Phi_{i} L^{i}
$$

existe, e os coeficientes matriciais de $\Phi(L)=A(L)^{-1}$ são absolutamente somáveis.

Demonstração. Se $|A(z)| \neq 0$ para $|z| \leq 1$, então $A(z)$ é não singular para $|z| \leq 1$, e a matriz $A(z)-1=\Phi(z)=\operatorname{Adj}[A(z)] /|A(z)|$ existe para $|z| \leq 1$. Notando que cada um dos elementos de $\Phi(z)$ é uma função racional de $z$, sem singularidades para $|z| \leq 1$, podemos então fazer uma expansão em séries de potências convergente para $\Phi(z)$ :

$$
\Phi(z)=\sum_{i=0}^{\infty} \Phi_{i} z^{i} \quad,|z| \leq 1
$$

Consequentemente $\Phi_{i} z^{i} \longrightarrow 0$ quando $i \rightarrow \infty$. Em particular, para $z=1, \Phi_{i} \longrightarrow 0$ quando $i \longrightarrow \infty$, o que resulta em que : 


$$
\Phi(1)=\sum_{i=0}^{\infty} \Phi_{i}<\infty \quad,
$$

somáveis.

os coeficientes matriciais de $\Phi(L)$ são absolutamente

Proposição 2.2 Seja um processo $\operatorname{VAR}(p)$ :

$$
\mathrm{A}(\mathrm{L}) \chi_{\mathrm{t}}=\rho+\varepsilon_{\mathrm{t}}
$$

onde $\chi_{t}$ é um vetor n-dimensional, $\varepsilon_{t}$ é um ruido branco $n$-dimensional de média 0 e matriz de covariância $\Sigma_{\varepsilon}$, é uma matriz de constantes n-dimensional e $A(L)$ é uma matriz polinomial. O processo (2.9) é estacionário causal se e somente se $A(L)$ é uma matriz polinomial com as raízes fora do círculo unitário.

\section{Demonstração.}

$\Rightarrow$ o processo (2.9) é estacionário causal.

Primeiro, mostraremos para $p=1$. Para $p=1$, o processo (2.9) fica :

$$
\left(I_{n}-A_{1} L\right) \chi_{t}=\rho+\varepsilon_{t}
$$

que, por substituição recursiva, pode ser escrito como :

$$
\chi_{t}=\left(I_{n}+A_{1}+\ldots+A_{1}^{j} \rho+A_{1}^{j-1} \chi_{t-j-1}+\sum_{i=0}^{j} A_{1}^{i} \varepsilon_{t-i}\right.
$$

Aplicando esperança em (2.11) obtem-se :

$$
E\left(\chi_{t}\right)=\left(I_{n}+A_{1}+\ldots .+A_{1}^{j} \rho+A_{1}^{j+1} E\left(\chi_{t-j-1}\right)+\sum_{i=0}^{j} A_{1}^{i} E\left(\varepsilon_{t-j}\right)\right.
$$

Como $\left\{\boldsymbol{x}_{\boldsymbol{t}}\right\}$ é estacionário, deve verificar-se que, na expressão (2.12) (ver apédice B, seção B.2), para j $\rightarrow \infty$,

(i) a expressão $\sum_{i=0}^{\infty} A_{1}^{i}$ convirja e,

(ii) $A_{1}^{j}$ tenda a 0 .

Assumindo que a matriz $A_{1}$ tem $n$ autovalores diferentes $\lambda_{i}$, ela pode ser escrita como (ver apéndice A, seção A.3) : 


$$
\mathrm{A}_{1}=\mathrm{P} \Lambda \mathrm{P}^{-1}
$$

onde $\mathrm{P}$ é uma matriz não singular de dimensão $\mathrm{n} \times \mathrm{n}$, e $\Lambda=\operatorname{diag}\left(\lambda_{1}, \ldots, \lambda_{n}\right\}$. Assim, tem-se :

$$
\mathrm{A}_{1}^{j}=\mathrm{P} \Lambda \mathrm{P}^{-1} \mathrm{P} \Lambda \mathrm{P}^{-1} \mathrm{P} \Lambda \mathrm{P}^{-1} \ldots \mathrm{P} \Lambda \mathrm{P}^{-1}=\mathrm{P} \Lambda \mathrm{P}^{-1}
$$

Ora,

$$
\lim _{j \rightarrow \infty} A_{1}^{j}=\lim _{j \rightarrow \infty} P \Lambda P^{-1}=P\left(\lim _{j \rightarrow \infty} \Lambda^{j}\right) P^{-1}=0
$$

se $\left|\lambda_{i}\right|<1$ para $i=1, \ldots, n$. Por outro lado, se isto é verdade, a sequência de matrizes em (i) é convergente :

$$
\sum_{j=0}^{\infty} A_{1}^{j}=\left(I_{n}-A_{1}\right)^{-1}
$$

Então, se o processo $(2.11)$ é estacionário, $\left|\lambda_{i}\right|<1$ para $i=1, \ldots, n$. Isto é equivalente a dizer, pela definição de autovalores (ver apéndice A, seção A.2), que as raízes do polinomio característico de $A_{1}, \quad\left|\lambda I_{n}-A_{1}\right|$, estão todas dentro do círculo unitário. E fácil ver que se isto é verdade, as raízes de $\left|I_{n}-A_{1} z\right|$ estão fora do círculo unitário.

Para $p>1$, o processo (2.9) pode ser escrito como um processo VAR(1) de dimensão np :

$$
Y_{t}=v+A Y_{t-1}+U_{t}
$$

onde :

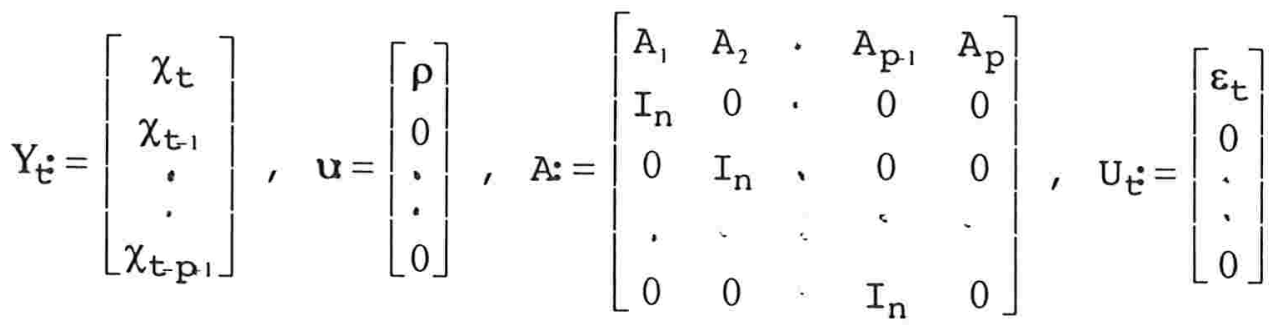

$$
\begin{aligned}
& \begin{array}{llll}
(\mathrm{np} \times 1) & (\mathrm{np} \times 1) & (\mathrm{np} \times \mathrm{np}) & (\mathrm{np} \times 1)
\end{array}
\end{aligned}
$$


Se o processo (2.17) é estacionário causal, então pela discussão acima :

$$
\left|I_{n p}-A z\right| \neq 0 \text { para }|z| \leq 1
$$

É fácil mostrar que :

$$
\left|I_{n p}-A z\right|=\left|I_{n}-A_{1} z-\ldots-A_{p} z\right|
$$

Assim, se (2.17) é um processo estacionário causal, por (2.19) e (2.20), temos :

$$
\left|I_{n p}-A z\right|=\left|I_{n}-A_{1} z-\ldots-A_{p} z\right| \neq 0 \text { para }|z| \leq 1
$$

$\Leftarrow$ As raízes de polinomio $A(L)$ em (2.9) estão todas fora do círculo unitário.

$A(L)$ é invertível pela proposição 2.1 , portanto fazendo $A(L)^{-1}=\Phi(L)$, e pre-multiplicando (2.8) por $\Phi(L)$, obtemos:

$$
\begin{aligned}
\chi_{t} & =\Phi(L) \rho+\Phi(L) \varepsilon_{t} \\
& =\left(\sum_{i=0}^{\infty} \Phi_{i}\right) \rho+\sum_{i=0}^{\infty} \Phi_{i} \varepsilon_{t-i}
\end{aligned}
$$

$\Phi(L)$ é absolutamente somável pela proposição 2.1 , e $\varepsilon_{\mathrm{t}}$ é uma variável aleatória de média e variância finitas. Como resultado os momentos de primeira e segunda ordens de ambos os termos de (2.9) são definidos (ver apêndice $B$, seção B.2), sendo então definidas a média e variância de $\boldsymbol{\chi}_{\mathbf{t}}$.

A média de $\chi_{t}$ é :

$$
\begin{aligned}
& E\left(\chi_{t}\right)=E(\Phi(L) \rho)+E\left(\Phi(L) \varepsilon_{t}\right) \\
& =\left(\sum_{i=0}^{\infty} \Phi_{i}\right) \rho+\sum_{i=0}^{\infty} \Phi_{i} E\left(\varepsilon_{t-i}\right) \\
& =\left(\sum_{i=0}^{\infty} \Phi_{i}\right) \rho=\mu\langle\infty
\end{aligned}
$$

o que verifica (i) na definição 2.2 . 
A autocovariância de $\chi_{t}$ com relação a $\chi_{t-h}$ é também

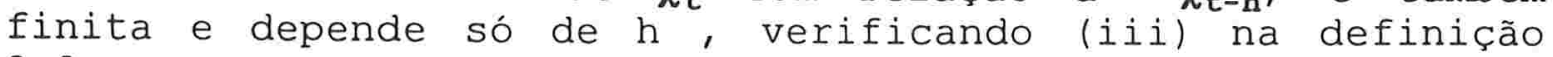
2.2 :

$$
\begin{aligned}
\Gamma_{x}(\mathrm{~h}) & =E\left(\chi_{t}-\mu\right)\left(\chi_{t-h}-\mu\right)^{\prime} \\
= & \left(\sum_{i=0}^{h-1} \Phi_{i} \varepsilon_{t-i}+\sum_{i=0}^{\infty} \Phi_{h \cdot i} \varepsilon_{t-h-i}\right)\left(\sum_{i=0}^{\infty} \Phi_{i} E\left(\varepsilon_{t-h i}\right)\right) \\
= & \sum_{i=0}^{\infty} \Phi_{h i} \sum_{\varepsilon} \Phi_{i}^{\prime}\langle\infty
\end{aligned}
$$

Também, por (2.11), verificamos (ii) na definição 2.2 :

$$
\begin{aligned}
\Sigma_{x} & =\Gamma_{x}(0)=E\left(x_{t}-\mu\right)\left(x_{t}-\mu\right)^{\prime} \\
& =\sum_{i=0}^{\infty} \Phi_{i} \Sigma_{\varepsilon} \Phi_{i}<\infty
\end{aligned}
$$

Assim, fica provada a proposição

\section{Observações :}

1) A forma (2.22) é chamada Representação de Média Móvel (MA), onde $\chi_{t}$ é expressado em termos dos valores passados e presentes do vetor $\varepsilon_{\mathrm{t}}$ e sua média $\mu$.

2) No futuro, quando falaremos de processos $\operatorname{VAR}(p)$ estacionários, estaremos nos referindo a processos estacionários causais. A seguir desenvolvemos um exemplo de processo "estacionário" não causal. Seja um AR(1), univariado :

$$
y_{t}=a_{1} y_{t-1}+\varepsilon_{t}
$$

onde $\left|a_{1}\right|>1$, ou seja, a raíz do polinomio $\left|1-a_{1} z\right|$ está dentro do círculo unitário, e onde naturalmente, não se 
verifica que $\sum_{j=0}^{\infty} a_{1}^{j}$ é um polinomio absolutamente somável. O processo (2.26) pode ser escrito como :

$$
y_{t}=a_{1}^{-1} y_{t+1}-a_{1}^{-1} \varepsilon_{t-1}
$$

que, após substituição recursiva, dá :

$$
y_{t}=-\sum_{i=1}^{\infty} a_{1}^{-i} \varepsilon_{t+i}
$$

Esta forma de representação, que é definida matemáticamente, é usualmente considerada como inaceitável. Típicamente, $\left\{\boldsymbol{x}_{\mathrm{t}}\right\}$ é considerado como função de $\left.\left\{\boldsymbol{\varepsilon}_{\mathrm{t}}, \mathrm{s} \leq \mathrm{t}\right\}\right\}$.

3) Os coeficientes $\Phi$ i em (2.6) são facilmente obtidos a partir de (2.4), identificando os coeficientes dos termos da mesma potência em :

$$
\begin{aligned}
I_{n} & =\left(\Phi_{0}+\Phi_{1} L+\ldots\right)\left(I_{n}-A_{1} L-\ldots-A_{p} L^{P}\right. \\
& =\Phi_{0}+\left(\Phi_{1}-\Phi_{0} A_{1}\right) L+\left(\Phi_{2}-\Phi_{1} A_{1}-\Phi_{0} A_{2}\right) L^{2}+\ldots+\left(\Phi_{i}-\sum_{j=1}^{i} \Phi_{i-j} A_{j} L^{i}+\ldots\right.
\end{aligned}
$$

onde $A_{j}=0$ para $j>p$. Assim, $\Phi_{i}$ pode ser obtido recursivamente usando-se :

$$
\begin{aligned}
& \Phi_{0}=I_{\mathbf{r}} \\
& \Phi_{i}=\sum_{j=1}^{i} \Phi_{i-j} A_{j}
\end{aligned}
$$

\subsubsection{Estimação de Modelos VAR(p) Estáveis}

\subsubsection{Estimação de Mínimos Quadrados Multivariada}

Seja dada uma série de tempo $\chi_{1}, \ldots, \chi_{T}$ ou seja, a disponibilidade de uma amostra de tamanho $\mathrm{T}$, para cada uma das $n$ variáveis. Além disso, suponha que existem $p$ valores pré-amostrais para cada variável $\chi_{-p+1}, \ldots \chi_{0}$. Particionando a série de tempo em valores amostrais e pré-amostrais de maneira conveniente, definimos :

$$
\mathrm{X}:=\left[\chi_{1}, \ldots, \chi_{\mathrm{T}}\right] \quad \mathrm{n} \times \mathrm{T}
$$


$B:=\left[\rho, A_{1}, \ldots, A_{p}\right] \quad n \times(n p+1)$

$\mathrm{z}_{\mathrm{t}^{\dot{2}}}=\left[\begin{array}{c}1 \\ \chi_{t} \\ \\ \chi_{t-p 1}\end{array}\right]$

$(n p+1) \times 1$

$$
\begin{array}{lc}
Z:=\left[\mathrm{Z}_{0}, \ldots, \mathrm{z}_{\mathrm{T}-1}\right] & (\mathrm{np}+1) \times \mathrm{T} \\
\mathrm{E}:=\left[\varepsilon_{1}, \ldots, \varepsilon_{\mathrm{T}}\right] & \mathrm{n} \times \mathrm{T}
\end{array}
$$

Usando esta notação para $t=1, \ldots \mathrm{T}$, o modelo (2.8) pode ser escrito de maneira compacta como :

$$
\mathrm{X}=\mathrm{BZ}+\mathrm{E}
$$

Pode-se demonstrar (Lutkepohl, 1993, págs.63-65) que o estimador de mínimos quadrados ordinários de B é dado por :

$$
\hat{\mathrm{B}}=X Z^{\prime}\left(Z Z^{\prime}\right)^{-1}
$$

onde a matriz de covariância de $\hat{B}$ estimada está dada por:

$$
\begin{aligned}
& \hat{\operatorname{var}}(\hat{B})=\left(Z Z^{\prime}\right)^{-1} \otimes \hat{\Sigma}_{\varepsilon} \\
& \hat{\Sigma_{\varepsilon}}=\frac{1}{T-m p-1} X\left(I_{T}-Z^{\prime}\left(Z Z^{\prime}\right)^{-1} Z\right) X^{\prime}
\end{aligned}
$$

Pode-se demonstrar que os estimadores (2.33) e (2.34) são não viesados e consistentes (Lutkepohl, 1993, págs.65-69).

É interessante notar o seguinte. O estimador de Mínimos Quadrados pode ser escrito de uma forma alternativa :

$$
\hat{\mathrm{b}}=\operatorname{vec}\left(\hat{\mathrm{B}^{\prime}}\right)=\left(I_{\mathrm{n}} \otimes\left(Z Z^{\prime}\right)^{-1} Z\right) \operatorname{vec}\left(X^{\prime}\right)
$$

onde vec denota o operador vetorização. Nesta notação é fácil ver que na verdade o estimador (2.33) equivale a 
calcular mínimos quadrados ordinários para cada uma das $\mathrm{n}$ equações separadamente.

\subsubsection{Outras Técnicas de Estimação}

O Estimador de Verossimilhança para B dá idênticos resultados aos de Mínimos Quadrados Ordinários Multivariado, portanto, possui as mesmas propriedades de ser não tendencioso e consistente, além de possuir distribuição normal asintóticamente.

Uma técnica interessante consiste na aplicação de Mínimos Quadrados Recursivos, que permite ver o comportamento do estimador de $\mathrm{B}$ ao longo do tempo. Para mais detalhes sobre esta técnica ver Harvey (1990, págs. 52-56).

\subsubsection{Modelos VARMA e Modelos MA de Ordem Finita}

O modelo VAR faz parte da versão mais geral do denominado modelo VARMA (Modelo Autoregressivo-Médias Móveis Vetorial). No modelos VARMA, $\boldsymbol{\varepsilon}_{\mathrm{t}}$ em (2.1) e (2.2) já não é mais um ruido branco ou inovação, mas apresenta uma estrutura de autocorrelação que em sua versão mais simples faz com que $\boldsymbol{\varepsilon}_{\mathrm{t}}$ tenha uma representação do tipo média móvel finita :

$$
\begin{aligned}
& \varepsilon_{t}=u_{t}+M_{1} u_{t-1}+\ldots+M u_{t-q} \\
& \text { ou } \\
& \varepsilon_{t}=M(L) u_{t}
\end{aligned}
$$

onde :

$u_{t}$ é um ruido branco, com $E\left(u_{t}\right)=0, E\left(u_{t} u_{t}{ }^{\prime}\right)=\Sigma$ e $E\left(u_{t} u_{s}{ }^{\prime}\right)=0$ para $t \neq s, e M(L)$ é definida como :

$$
\mathrm{M}(\mathrm{L})=\mathrm{I}_{\mathrm{n}}+\mathrm{M}_{1} \mathrm{~L}+\ldots+\mathrm{M}_{q} \mathrm{~L}
$$

Assim, temos a seguinte definição :

Definição 2.40 modelo representado pela expressão :

$$
\mathrm{A}(\mathrm{L}) \chi_{\mathrm{t}}=\rho+\mathrm{M}(\mathrm{L}) \mathrm{u}_{\mathrm{t}}
$$

onde $A(L)$ e $M(L)$ são definidas como em (2.3) e (2.37), é denominado modelo VARMA $(p, q)$, ou Modelo AutoregressivoMédias Móveis Vetorial. 
Note que no Modelo $\operatorname{VAR}(p)$, no contexto do modelo VARMA definido acima, $M(L)$ é simplesmente uma matriz identidade.

Proposição 2.3 Seja o processo estocástico $\operatorname{VARMA}(p, q)$ definido na expressão (2.38). Se todas as raízes do polinômio matricial $A(L)$ estão fora do círculo unitário, $A(L)$ é invertível, $O$ que implica que $O$ processo é estacionário.

Demonstração. Ver Brockwell \& Davis (1991, pág. 408), e a demonstração da proposição 2.2 .

A partir de (2.38), multiplicando a direita por $A(L)^{-1}=: \Phi(L) \quad$ (se $A(L)$ é invertível), pode ser obtida uma representação puramente MA de ordem infinita :

$$
\begin{aligned}
& A(L) \chi_{t}=\rho+M(L) \varepsilon_{t} \\
& \begin{aligned}
\chi_{t} & =\Phi(L) \rho+\Phi(L) M(L) \varepsilon_{t} \\
& =\left(\sum_{i=0}^{\infty} \Phi_{i}\right) \rho+\sum_{i=0}^{\infty} \Lambda_{i} \varepsilon_{t-i} \\
& =\mu+\sum_{i=0}^{\infty} \Lambda_{i} \varepsilon_{t-i}
\end{aligned}
\end{aligned}
$$

onde $\Phi(\mathrm{L}) \mathrm{M}(\mathrm{L})=\sum_{\mathrm{i}=0}^{\infty} \Lambda_{i} \varepsilon_{\mathrm{t}-\mathrm{i}}$, sendo que esta última expressão converge.

Definição 2.5 Seja o processo $\operatorname{VARMA}(p, q)$ definido na expressão (2.38). Se todas as raízes do polinômio matricial $M(L)$ estão fora do círculo unitário, $M(L)$ tem inversa, e o processo é chamado de invertível.

Se um processo VARMA $(p, q)$ é invertível, pode ser escrito como um processo VAR de ordem infinita :

$$
\begin{aligned}
& \mathrm{A}(\mathrm{L}) \chi_{t}=\rho+\mathrm{M}(\mathrm{L}) \varepsilon_{t} \\
& \mathrm{M}(\mathrm{L})^{-1} \mathrm{~A}(\mathrm{~L}) \chi_{t}=\mathrm{M}\left(\mathrm{L}^{-1} \rho+\varepsilon_{t}\right. \\
& \Pi(\mathrm{L}) \chi_{t}=\mathrm{M}(\mathrm{L})^{-1} \rho+\varepsilon_{t}
\end{aligned}
$$

onde 


$$
M(L)^{-1} A(L)=\Pi(L)=I_{n}-\sum_{i=1}^{\infty} \Pi_{i}
$$

é uma sequência de matrizes convergente.

Quando $A(L)$ em (2.38) é uma matriz identidade, obtemos o denominado Modelo MA ou Modelo de Média Móvel Vetorial.

Definição 2.60 modelo representado pela expressão :

$$
\chi_{t}=\rho+M(L) u_{t}
$$

onde $M(L)$ corresponde à definição dada em (2.37), é denominado Modelo MA(q) ou Modelo de Média Móvel Vetorial de ordem $q$.

Se as raízes do polinômio $M(L)$ estão todas fora do círculo unitário, então temos a presença de um processo MA invertível, que pode ser escrito como um processo VAR de ordem infinita.

\subsection{A Representação MCE - Mecanismo de Correção de Erro}

\subsubsection{Integração e Co-integração}

Seja $\left\{\boldsymbol{\chi}_{\mathrm{t}}\right\}$ um processo $\mathrm{AR}(\mathrm{p})$ do tipo da expressão (2.2), não estacionário, onde $\chi_{t}$ é um vetor $n \times 1$. Sendo que o processo é não estacionário, existem algumas raízes do polinômio matricial A(L) que estão sobre o círculo unitário, assumindo que todas as outras raízes estão fora do círculo unitário (não consideramos o caso onde o polinômio $A(L)$ tem raízes dentro do cículo unitário, que corresponde ao caso explosivo). Suponha que existem d raízes unitárias. Multiplicando à direita pela matriz adjunta de $A(L)$, este processo pode ser escrito como :

$$
\begin{aligned}
& \mathrm{A}(\mathrm{L}) \chi_{t}=\rho+\varepsilon_{t} \\
& |\mathrm{~A}(\mathrm{~L})| \chi_{\mathrm{t}}=\mathrm{A}(\mathrm{L})^{\circ} \rho+\mathrm{A}(\mathrm{L})^{\circ} \varepsilon_{\mathrm{t}}
\end{aligned}
$$


onde $A(L)^{\bullet}$ é a matriz adjunta de $A(L)$.

O i-ésimo componente de $\boldsymbol{\chi}_{t}$ tem a representação :

$$
|\mathrm{A}(\mathrm{L})| \chi_{\text {it }}=\overline{\mathrm{r}}_{\mathrm{i}}+\overline{\mathrm{m}}_{\mathrm{i}}(\mathrm{L}) \mathrm{u}_{\text {i上 }}
$$

onde $v_{i t}$ é um ruido branco.

Se $|A(L)|$ tem d raízes unitárias, sendo que as outras estão fora do círculo unitário, a expressão (2.44) pode ser escrita como :

$$
\alpha(L)(1-L)^{d} \chi_{i t}=\alpha(L) \Delta^{d} \chi_{t}=\bar{r}_{i}+\bar{m}_{i}(L) v_{\text {it }}
$$

onde $\alpha(L)$ é um operador invertível. Consequentemente, $\Delta^{\mathrm{d}} \boldsymbol{\chi}_{\text {it }}$ é um processo estacionário. Em outras palavras, se o processo VAR(p) é não estacionário, cada um dos componentes do vetor $\chi_{t}$ pode se tornar estacionário após ter sido diferenciado d vezes. Note, no entanto, que quando o vetor é considerado conjuntamente, pode não ser necessário diferenciar d vezes devido a cancelamentos. Para ilustrar esse ponto considere o processo :

$$
\left(\left[\begin{array}{ll}
1 & 0 \\
0 & 1
\end{array}\right]-\left[\begin{array}{ll}
1 & 0 \\
0 & 1
\end{array}\right] \mathrm{L}\right)\left[\begin{array}{l}
\chi_{1 t} \\
\chi_{2 t}
\end{array}\right]=\left[\begin{array}{l}
\varepsilon_{1 t} \\
\varepsilon_{2 t}
\end{array}\right]
$$

Em (2.46) temos que :

$$
|A(L)|=\left[\begin{array}{cc}
1-L & 0 \\
0 & 1-L
\end{array}\right]=(1-L)^{2}
$$

tem duas raízes unitárias. No entanto, é óbvio que o vetor $\chi_{t}$ torna-se estacionário após uma diferenciação.

Os comentários feitos nas linhas anteriores podem facilmente ser estendidos para os processos ARMA $(p, q)$ não estacionários, os quais podem ser transformados em processos estacionários após um número conveniente de diferenciações. Assim temos a seguinte definição : 
Definição 2.7 (Granger, 1981): Uma série sem componente determinístico com uma representação ARMA estacionária e invertível, após ter sido diferenciada d vezes, é dita integrada de ordem $d$, denotada por $y_{t} \sim I(d)$.

Nesta definição e notação, por exemplo, uma série qualquer dita $I(0)$ é estacionária; se a série é $I(1)$, isto significa que a primeira diferença será estacionária.

A definição 2.7 pode ser estendida a uma série temporal vetorial, ou seja, podemos trabalhar com um vetor $\chi_{t}$ cujos componentes sejam todos $I(d)$, e dizemos que $\chi_{t} \sim I(d)$.

Agora, um vetor $x_{t} n$-dimensional, embora tendo todos os seus componentes $I(d)$ com $d>0$, ou seja, não estacionários, pode apresentar combinações lineares de seus componentes que sejam estacionários. Introduzimos agora a definição de cointegração.

Definição 2.8 (Engle and Granger, 1987; Johansen, 1988) : Os componentes do vetor $\chi_{t}$ são ditos co-integrados de ordem $d, b$, denotado por $\chi_{t} \sim C I(d, b)$, se

(i) todos os componentes de $\chi_{t}$ são I(d);

(ii) existe uma matriz $\alpha(\neq 0)$ de dimensão $n \times r$ tal que $z_{t}=\alpha^{\prime} \chi_{t} \sim I(d-b), b>0$. Os vetores que compoem $\boldsymbol{\alpha}$ são chamados de vetores co-integrados.

Por exemplo, para um vetor $\chi_{t} \sim I(1,1)$ de dimensão $n \times 1, a$ definição 2.8 implica que $\left\{\chi_{t}\right\}$ não é estacionário, mas $\left\{x_{t}-x_{t-1}\right\}$ é estacionário. No entanto, existem combinações lineares dos componentes de $\chi_{t}$ que são estacionários. Considere, por exemplo um vetor cujos componentes são Renda $\left(y_{t}\right)$ e Consumo $\left(c_{t}\right)$ no Brasil para um período de 20 anos, expressados en logaritmos. Tipicamente, estas séries são não estacionárias. No entanto, hipoteticamente, o consumo pode guardar uma relação fixa com a renda, por exemplo $C_{t} / Y_{t}=e^{k}$, com o qual $\ln \left(C_{t}\right)-\ln \left(y_{t}\right)=c_{t}-y_{t}=k$. Aqui $\alpha=\left[\begin{array}{ll}1 & -1\end{array}\right]$.

\subsubsection{A Representação MCE - Mecanismo de Correção de Erro}

Seja $\chi_{t}$ um vetor $I(1)$, de dimensão $n \times 1$, cujos componentes são CI $(1,1)$ com $r$ vetores co-integrados, representado por um modelo $\operatorname{VAR}(p)$ de ordem finita :

$$
A(L) \chi_{t}=\rho+\rho^{*} D_{t}+\varepsilon_{t}
$$


onde :

$$
A\left(I_{1}\right)=I_{n}-\sum_{j=1}^{p} A_{j} I^{j},
$$

$\rho$ e $\rho^{*}$ são vetores $n \times 1$ de constantes, $D_{t}$ é um escalar que representa uma variável dummy e $\left\{\varepsilon_{\mathrm{t}}\right\}$ é um ruido branco com $E\left\{\varepsilon_{t}\right\}=0$ e $E\left\{\varepsilon_{t} \varepsilon_{t}{ }^{\prime}\right\}=\Sigma$, uma matriz positiva definida.

Defina $A(L)$ como :

$$
A(L)=: A^{*}(L)(1-L)+A(1) L
$$

onde

$$
A(l)=I_{n}-\sum_{j=1}^{p} A_{j}
$$

Pode ser demonstrado (ver apêndice C) que :

$$
A^{*}(L)=I_{n}-\sum_{i=1}^{p-1} A_{i}^{*} L^{i} \quad \text { onde } \quad A_{i}^{*}=-\sum_{j=i+1}^{p} A_{j}
$$

Substituindo $(2.50)$ em (2.48) temos :

$$
\begin{aligned}
& {\left[A^{*}(L)(1-L)+A(1) L\right] x_{t}=\rho+\rho^{*} D_{t}+\varepsilon_{t}} \\
& A^{*}(L) \Delta \chi_{t}+A(1) \chi_{t-1}=\rho+\rho D_{t}+\varepsilon_{t}
\end{aligned}
$$

o que permite reescrever (2.48) como:

$$
A^{*}(\mathrm{~L}) \Delta \chi_{t}=\rho+\rho^{*} D_{t}-A(l) \chi_{t-1}+\varepsilon_{t}
$$

onde $\Delta:=1-\mathrm{L}$ é o operador de primera diferença.

Como $\chi_{t}$ é integrada de ordem um, $|A(L)|$ possui $n$ raízes unitárias. Portanto $|\mathrm{A}(1)|=0$, implicando que a matriz 
polinomial n-dimensional $A(1)$, tem posto inferior $a n$. Suponha que :

$$
\text { posto }[A(1)]=r, \quad 0<r<n
$$

Então a matriz $A(1)$ pode ser escrita como o produto de duas matrizes $\boldsymbol{\gamma}$ e $\boldsymbol{\alpha}$ de dimensão $\mathrm{n} \times \mathrm{r}^{1}$ :

$$
A(1)=\gamma \alpha^{\prime}
$$

Por outro lado, segue de (2.53) que [A(1) $\left.\chi_{t-1}\right]$ deve ser estacionário, sendo que o lado esquerdo da equação (2.53) denota um vetor estacionário. Então, pode ser escrito como função linear de um vetor estacionário. Por (2.55) temos :

$$
\mathrm{A}(\mathrm{l}) \chi_{\mathrm{t}-1}=\gamma \alpha^{\prime} \chi_{\mathrm{t}-1}=\gamma z_{\mathrm{t}-1}
$$

Portanto $\boldsymbol{\alpha}^{\prime} \boldsymbol{\chi}_{\mathrm{t}}=\boldsymbol{z}_{\mathrm{t}}$ é estacionário, e $\boldsymbol{\alpha}$, conforme a definição 2.6, é a matriz dos vetores co-integrados, representado $r$ relações de co-integração. Assim, $\chi_{t} \sim C(1,1)$, com $r$ vetores co-integrados.

Note que se posto $[A(1)]=0$, não temos vetores cointegrados, e a expressão (2.53) se reduz a um modelo de primeiras diferenças AR com desvío ('drift"), isto é, a representação válida é VAR em diferenças. Por outro lado se posto $[A(1)]=n$, não há co-integração, mas todas as variáveis são estacionárias; a representação válida é a de $\operatorname{VAR}(p)$ em níveis.

Substituindo (2.56) em (2.53), obtemos a chamada representação de Mecansimo de Correção de Erro (MCE) :

$$
\begin{aligned}
A^{*}(\mathrm{~L}) \Delta \chi_{t} & =\rho+\rho^{*} D_{t}-\gamma \alpha^{\prime} \chi_{t-1}+\varepsilon_{t} \\
& =\rho+\rho^{*} D_{t}-\gamma z_{t-1}+\varepsilon_{t}
\end{aligned}
$$

que consiste simplesmente em ressaltar a restrição que colocamos em $A(1)$, com relação ao posto desta matriz. Nesta representação, as variáveis explicativas são o passado de $\Delta \boldsymbol{x}_{t}$, que representa os efeitos de curto prazo², e o

\footnotetext{
${ }^{1}$ Note que, para $r>1$, as matrizes $\alpha$ e $\gamma$ não são únicas. Se $r=1, \alpha$ é único a menos de sinal.

${ }^{2}$ No caso de exogeneidade fraca de alguma/algumas variáveis do sistema, o presente de $\Delta \chi_{t}$ também pode se tornar variável explicativa no sistema. Ver Ericsson, N. R., 1992
} 
desequilíbrio entre componentes do vetor $x_{t}$ no periodo anterior, considerando a relação de equilíbrio existente no longo prazo (Engle and Granger, 1987).

Como $A(1)$ é uma matriz singular, técnicas padrões para inverter a forma (2.53) não podem ser utilizadas para obter a representação de Wold, que é um passo necessário para a obtenção da representação de Tendências Comuns, como veremos mais adiante ${ }^{3}$. No entanto, a partir da estimação dos parâmetros da representação $\mathrm{MCE}$, podemos obter uma representação alternativa, denominada VAR "restrita", que nos permitirá obter com maior facilidade a representação de Wold, como será explicado mais detalhadamente nos próximos capítulos.

\subsubsection{Estimação de Modelos MCE}

Para estimar (2.53), o método de Johansen (1988, 1989, 1991), baseado na função de verossimilhança da amostra, pode ser usado. Este método calcula A(1) - e permite testar sobre o posto de $A(1)$-, os vetores $\alpha$ e $\gamma$, de onde derivaremos os parâmetros da polinomial $A^{*}(\mathrm{~L})$ e o resto dos parâmetros. Básicamente, o método calcula a matriz $\alpha$, considerando os quadrados das correlações canônicas de $\mathrm{R}_{1 \mathrm{t}}$ com relação a $\mathrm{R}_{\text {ot }}$ ' onde $R_{\text {ot }}$ são os resíduos da regressão de $\Delta \chi_{t}$ sobre as diferenças desfasadas $\Delta \chi_{t-1}, \ldots, \Delta \chi_{t-p+1}$, e $R_{1 t}$ são os da regressão de $\chi_{t-1}$ sobre as diferenças $\Delta \chi_{t-1}, \ldots, \Delta \chi_{t-p+1}$. Os vetores de $\alpha$ são as $r$ maiores variáveis canônicas. Para testar sobre a dimensão do espaço de co-integração, i.e. o posto da matriz $A(1)$, são calculadas a estatística Lambdamax e a estatística do Traço. Estas estatísticas são usadas para testar sequêncialmente $H_{0}$ : Posto[A(1)] $=r_{0}$ vs $H_{a}$ : Posto $[A(1)]=r_{1}>r_{0}$.

Os estimadores dos coeficientes do polinomio $A^{*}(L)$, das constantes, dos coeficientes das variáveis exógenas e de $\gamma \alpha^{\prime}$ tem as propiedades asintóticas usuais, se $\boldsymbol{\chi}_{t}$ é um processo $\operatorname{VAR}(\mathrm{p})$ Gaussiano. A estimação consistente de $\gamma$ e $\alpha$ separadamente requere restrições adicionais, sendo que existe um problema de identificação (lembre que $\gamma$ e $\alpha$ podem asumir diferentes valores, dependendo da normalização realizada). O método de Johansen é discutido com mais detalhes no apêndice $D$.

Engle \& Granger (1987) propuseram um estimador em dois passos, quando existe um só vetor de co-integração. No

\footnotetext{
${ }^{3}$ Um algoritmo para obter a representação de Wold partindo da representação do MCE é descrita em Lutkepohl \& Reimers (1992).
} 
primeiro passo, os componentes do vetor $\alpha$ são estimados, fazendo uma regressão em níveis das variáveis. No segundo passo, estes parâmetros são usados na representação de MCE (2.41), e o resto dos parâmetros é estimado. Nos dois passos, mínimos quadrados ordinários são usados. Note que as estimativas dos parâmetros no primeiro passo convergem a suas probabilidades limite à taxa $T$ (são superconsistentes), sendo que os parâmetros no segundo passo convergem à taxa asintótica usual $\mathrm{T}^{1 / 2}$. Existem dois problemas com a estimação do vetor co-integrado neste método : a) a forte possibilidade de não indepêndencia entre os erros e os regressores; b) forte possibilidade de regressões espúreas. O primeiro problema tende a diminuir asintóticamente, e o segundo problema também, se os componentes do vetor são realmente co-integrados. No entanto, para pequenas amostras, as estimativas podem ser viesadas (ver Davidson e Mac Kinnon, pág. 719).

De acordo com um teorema demonstrado por Engle \& Granger (1987), o estimador em dois passos de uma equação dentro de um sistema de Correção de Erro com um só vetor de cointegração, obtido a partir de uma estimativa de $\boldsymbol{\alpha}$ em uma regressão estática das variáveis do sistema, em vez do valor verdadeiro de $\alpha$, terá a mesma distribuição limite que o estimador de máxima versosimilhança usando o valor verdadeiro de $\alpha$.

Alternativamente, em vez de estimar $\alpha$, podemos fazer restrições, a priori, sobre todos ou alguns vetores de $\boldsymbol{\alpha}$, de acordo com a teoria econômica. Se o espaço de co-integração (a matriz $\alpha$ ) é conhecido, podemos então estimar o resto dos parâmetros usando mínimos quadrados ordinários.

\subsection{Exemplo}

A seguir, apresentamos um exemplo com o objetivo de esclarecer os conceitos e mecanismos introduzidos nas seções 2.1 e 2.2 deste capítulo.

Seja $\boldsymbol{\chi}_{\mathrm{t}}$ um vetor, de dimensão $3 \times 1$, tal que $\boldsymbol{\chi}_{\mathrm{t}} \sim \mathrm{CI}(1,1)$, e $r=1$, ou seja, existe uma matriz $\alpha$, de dimensão $n \times 1$ (um vetor co-integrado), tal que $\alpha^{\prime} \chi_{t} \sim I(0)$. Suponha que $\chi_{t}$ segue um processo VAR(2), e que $D_{t}$ é uma variável dummy escalar que assume os valores 0 ou 1 . A representação de $\chi_{t}$ na representação $A R(2)$ será : 


$$
\left[I_{3}-A_{1} L-A_{2} L^{2}\left[\begin{array}{l}
\chi_{1 t} \\
\chi_{2 t} \\
\chi_{3 t}
\end{array}\right]=\left[\begin{array}{l}
\rho_{1} \\
\rho_{2} \\
\rho_{3}
\end{array}\right]+\left[\begin{array}{l}
\rho_{1}^{*} \\
\rho_{2}^{*} \\
\rho_{3}^{*}
\end{array}\right] D_{t}+\left[\begin{array}{c}
\varepsilon_{1 t} \\
\varepsilon_{2 t} \\
\varepsilon_{3 t}
\end{array}\right]\right.
$$

onde :

$$
A_{1}=\left[\begin{array}{lll}
a_{11,} & a_{12,} & a_{13,} \\
a_{21,} & a_{22,} & a_{23,} \\
a_{31,} & a_{32,} & a_{33,}
\end{array}\right] \quad \text { e } \quad A_{2}=\left[\begin{array}{lll}
a_{11,2} & a_{12,} & a_{13,2} \\
a_{21,2} & a_{22,2} & a_{23,2} \\
a_{31,2} & a_{32,2} & a_{33,}
\end{array}\right]
$$

Agora: 。

$$
A^{*}(L)=I_{3}-\sum_{i=1}^{1}\left(-\sum_{j=i+1}^{2} A_{j} L^{i}=I_{3}+A_{2} L\right.
$$

e

$$
A(l)=I_{3}-A_{1}-A_{2}
$$

Então, a representação baseada em (2.57) do processo (2.58) está dada por:

$$
\left[I_{3}+A_{2} L\left[\begin{array}{c}
\Delta \chi_{1 t} \\
\Delta \chi_{2 t} \\
\Delta \chi_{3 t}
\end{array}\right]=\left[\begin{array}{l}
\rho_{1} \\
\rho_{2} \\
\rho_{3}
\end{array}\right]+\left[\begin{array}{c}
\rho_{1}^{*} \\
\rho_{2}^{*} \\
\rho_{3}^{*}
\end{array}\right] D_{t}-\left(I_{3}-A_{1}-A_{2}\right)\left[\begin{array}{l}
\chi_{1 t-1} \\
\chi_{2 t-1} \\
\chi_{3 t-1}
\end{array}\right]+\left[\begin{array}{l}
\varepsilon_{1 t} \\
\varepsilon_{2 t} \\
\varepsilon_{3 t}
\end{array}\right]\right.
$$

ou: 


$$
\left[\begin{array}{l}
\Delta \chi_{1 t} \\
\Delta \chi_{2 t} \\
\Delta \chi_{3 t}
\end{array}\right]=\left[\begin{array}{l}
\rho_{1} \\
\rho_{2} \\
\rho_{3}
\end{array}\right]+\left[\begin{array}{l}
\rho_{1}^{*} \\
\rho_{2}^{*} \\
\rho_{3}^{*}
\end{array}\right] D_{t}-A_{2}\left[\begin{array}{l}
\Delta \chi_{1 t-1} \\
\Delta \chi_{2 t-1} \\
\Delta \chi_{3 t-1}
\end{array}\right]-\left[I_{3}-A_{1}-A_{2}\left[\begin{array}{l}
\chi_{1 t-1} \\
\chi_{2 t-1} \\
\chi_{3 t-1}
\end{array}\right]+\left[\begin{array}{l}
\varepsilon_{1 t} \\
\varepsilon_{2 t} \\
\varepsilon_{3 t}
\end{array}\right]\right.
$$

Como $\Delta x_{t}$ é estacionária, $A(1) x_{t-1}$ tambén deve ser estacionária, em consequência, função linear de $\alpha \chi_{t-1}$. Sendo que $r=1$, temos que $A(1)=\gamma \alpha^{\prime}$, onde $\gamma$ e $\alpha^{\prime}$ são vetores $3 \times 1$ :

$$
A(l)=I_{3}-A_{1}-A_{2}=\left[\begin{array}{c}
\gamma_{11} \\
\gamma_{21} \\
\gamma_{31}
\end{array}\right]\left[\begin{array}{lll}
\alpha_{11} & \alpha_{21} & \alpha_{31}
\end{array}\right]
$$

Note que o conhecimento de (2.61) implica em restrições em $A_{1}$ e $A_{2}$ na representação (2.58).

A representação de MCE fica :

$$
\left(I_{3}+A_{2} L\right)\left[\begin{array}{c}
\Delta \chi_{1 t} \\
\Delta \chi_{2 t} \\
\Delta \chi_{3 t}
\end{array}\right]=\left[\begin{array}{l}
\rho_{1} \\
\rho_{2} \\
\rho_{3}
\end{array}\right]+\left[\begin{array}{c}
\rho_{1}^{*} \\
\rho_{2}^{*} \\
\rho_{3}^{*}
\end{array}\right] D_{t}-\left[\begin{array}{l}
\gamma_{11} \\
\gamma_{21} \\
\gamma_{31}
\end{array}\right]\left[\alpha_{11} \chi_{1 t-1}+\alpha_{21} \chi_{2 t-1}+\alpha_{31} \chi_{3 t-1}\right]+\left[\begin{array}{c}
\varepsilon_{1 t} \\
\varepsilon_{2 t} \\
\varepsilon_{3 t}
\end{array}\right]
$$




\section{Capítulo 3}

\section{A Representação VAR "Restrita"}

Neste capítulo abordamos a chamada representação VAR "restrita", no sentido de que colocaremos certas restrições nos componentes de um modelo VAR a ser estimado (o VAR restrito), derivados da co-integração existente nos componentes do vetor estocástico em estudo. A representação VAR restrita pode ser obtida a partir das estimativas dos parâmetros no MCE, ou por técnicas padrões para a estimação de um VAR estacionário, se os vetores co-integrados (a matriz $\alpha$ ) são conhecidos. As estimativas obtidas permitirão obter estimativas dos parâmetros na representação de Wold, que será tratada no capítulo seguinte. Estes ítens são tratados na seção 3.1 .

No final de capítulo, na seção 3.2, um exemplo é apresentado para esclarecer a aplicação dos procedimentos.

\subsection{A Representação VAR "Restrita"}

Campbell e Shiller (1988) demonstram como de modo direto a representação $\mathrm{MCE}$ pode ser reescrita como uma representação VAR restrita quando $n=2$ e $r=1$. O teorema 1 em Warne (1991) mostra que este resultado pode ser generalizado.

Proposição 3.1

(i) Seja $\left\{\boldsymbol{x}_{t}\right\}$ um processo $A R(p)$ não estacionário :

$$
\mathrm{A}(\mathrm{L}) \chi_{t}=\rho+\rho^{*} D_{t}+\varepsilon_{t}
$$

onde $\chi_{t}$ é um vetor $n \times 1, \rho$ e $\rho^{*}$ são vetores $n \times 1$ de constantes, $D_{t}$ é um escalar que representa uma variável dummy e $\left\{\boldsymbol{\varepsilon}_{\mathrm{t}}\right\}$ é um ruido branco $\operatorname{com} E\left\{\boldsymbol{\varepsilon}_{\mathrm{t}}\right\}=0$ e $E\left\{\boldsymbol{\varepsilon}_{\mathrm{t}} \boldsymbol{\varepsilon}_{\mathrm{t}}{ }^{\prime}\right\}=\Sigma$, uma matriz positiva definida.

(ii) Assuma que $\chi_{t}$ possui $r$ vetores co-integrados, tendo então a representação MCE :

$$
\mathrm{A}^{*}(\mathrm{~L}) \Delta \chi_{t}=\rho+\rho^{*} \mathrm{D}_{\mathrm{t}}-\gamma \alpha^{\prime} \chi_{\mathrm{t}-1}+\varepsilon_{t}
$$


onde $\boldsymbol{\alpha}$ e $\boldsymbol{\gamma}$ são matrizes de dimensão $n \times r$, e $A^{*}(L)$ é um polinômio matricial onde

$$
A^{*}(L)=I_{n}-\sum_{i=1}^{p-1} A_{i}^{*} L^{i} \text { onde } A_{i}^{*}=-\sum_{j=i+1}^{p} A_{j}
$$

(iii) Defina as seguintes matrizes :

(iii.1) $M=\left[S_{k}{ }^{\prime} \alpha\right]^{\prime}$, uma matriz não singular de dimensão $n \times n$, onde $\mathrm{S}_{\mathrm{k}}$ é de dimensão $\mathrm{k} \times \mathrm{n}$, com $\mathrm{k}=\mathrm{n}-\mathrm{r}$;

(iii.2) $\gamma^{*}=[0 \gamma]$, uma matriz, de dimensão $n \times n$;

(iii.3) $D(L)$ e $D_{\perp}(L)$, matrizes polinomiais, de dimensão $\mathrm{n} \times \mathrm{n}$, dadas por:

$$
D(L)=\left[\begin{array}{cc}
I_{k} & 0 \\
0 & \left(1-L_{)} I_{r}\right.
\end{array}\right] \quad \text { e } \quad D_{\perp}(L)=\left[\begin{array}{cc}
\left(1-L_{)} I_{k}\right. & 0 \\
0 & I_{r}
\end{array}\right]
$$

observando que $D(L) D_{1}(L)=D_{1}(L) D(L)=I_{n}(1-L)$.

O processo $\left\{\chi_{t}\right\} \sim C I(1,1)$, com $r$ vetores co-integrados se e somente se existe uma representação VAR restrita, da forma :

$$
B(L) y_{t}=\theta+\theta^{*} D_{t}+\eta_{t}
$$

onde :

$$
\begin{gathered}
\mathrm{B}(\mathrm{L})=\mathrm{M}\left[\mathrm{A}^{*}(\mathrm{~L}) \mathrm{M}^{-1} \mathrm{D}(\mathrm{L})+\gamma^{*} \mathrm{~L}\right] \\
\mathrm{Y}_{\mathrm{t}^{*}}=\mathrm{D}_{\perp}(\mathrm{L}) \mathrm{M} \mathrm{X}_{\mathrm{t}}
\end{gathered}
$$

e

$$
\theta=\mathrm{M} \rho, \quad \theta^{*}=\mathrm{M} \rho *, \quad \eta_{\mathrm{t}}=\mathrm{M} \varepsilon_{\mathrm{t}}
$$

de modo que $\left\{\mathrm{y}_{\mathrm{t}}\right\}$ é um processo $\operatorname{VAR}(\mathrm{p})$ estacionário. Os últimos $r$ elementos de $y_{t}$ são dados pelas $r$ relações de cointegração.

Demonstração. Ver Warne (1991), para uma demonstração formal. A seguir demonstraremos informalmente a proposição anterior. 
Partindo de (3.1), pre-multiplique esta expressão pela matriz $M$, e pre-multiplique $x_{t}$ no primeiro membro de (3.1) por $\mathrm{MM}^{-1}$, obtendo :

$$
M A(L) M^{-1} M X_{t}=M p+M p D_{t}^{*}+M E_{t}
$$

Como $A\left(L_{2}\right)=A\left(L_{1}\right)^{*}(1-L)+A(l) L, \quad$ o primeiro membro da equação (3.8) fica :

$$
\begin{aligned}
\operatorname{MA}(\mathrm{L}) \mathrm{M}^{-1} \mathrm{M} \chi_{t} & =\mathrm{M}\left[\mathrm{A}(\mathrm{L})^{*}(1-\mathrm{L})+\mathrm{A}(\mathrm{l}) \mathrm{L}\right] \mathrm{M}^{-1} \mathrm{M} \chi_{t} \\
& =\mathrm{M}\left[\mathrm{A}(\mathrm{L})^{*} \mathrm{M}^{-1}(1-\mathrm{L})+\mathrm{A}(\mathrm{l}) \mathrm{M}^{-1} \mathrm{~L}\right] \mathrm{M} \chi_{t}
\end{aligned}
$$

Observe que :

$$
\begin{gathered}
\gamma^{*}=\gamma * D_{\perp}(L), \\
A(1)=\gamma \alpha^{\prime}=\left[0_{n \times k} \gamma\right]\left[S_{k}{ }^{\prime} \alpha\right]^{\prime}=\gamma^{*} M=\gamma * D_{\perp}(L) M e \\
I_{n}(1-L)=D(L) D_{\perp}(L) .
\end{gathered}
$$

Assim, substituindo (3.10) em (3.9), obtemos :

$$
\begin{aligned}
M A(L) M^{-1} M X_{t} & \left.=M[A(L))^{*} M^{-1} D(L) D_{\perp}(L)+\gamma^{*} D_{\perp}(L) M M^{-1} L\right] M X_{t} \\
& =M\left[A(L){ }^{*} M^{-1} D(L)+\gamma^{*} L\right] D_{\perp}(L) M X_{t} \\
& =B(L) Y_{t}
\end{aligned}
$$

Note que pela definição de $Y_{t}$, os primeiros $k$ componentes deste vetor são combinações lineares das primeiras diferenças dos componentes de $\chi_{t}$, em consequência estacionários. Os $r$ componentes restantes são os $r$ vetores co-integrados, também estacionários. Assim $Y_{t}$ é estacionário.

Finalmente, por (3.8), denominando $\theta=\mathrm{M} \rho, \theta^{*}=\mathrm{M} \rho^{*} \mathrm{e} \eta_{\mathrm{t}}=\mathrm{M} \varepsilon_{\mathrm{t}}$, fica provada a proposição.

Para a escolha dos $k$ primeiros elementos de $Y_{t}$, ou seja, para a escolha de $\mathrm{S}_{\mathrm{k}}$, temos duas alternativas :

1) deixar $S_{k}=\alpha^{\prime}{ }_{\perp}$ onde $\boldsymbol{\alpha}_{\perp}^{\prime} \boldsymbol{\alpha}=0$. Isto garante que $M$ seja não singular (ver apêndice $A$, seção A.1). 
2) Normalizar $\alpha$ de tal modo que $\alpha^{\prime}=\left[\alpha^{*} I_{r}\right]$, e deixando $S_{k}=\left[I_{k} 0\right]$, o que determina que os primeiros $k$ elementos de $Y_{t}$ sejam dados pelas primeiras diferenças dos primeiros $\mathrm{k}$ elementos de $\chi_{t}$. Note que $S_{k}=\left[\begin{array}{ll}I_{k} & 0\end{array}\right]$ somente é permissível se as últimas $r$ linhas de $\alpha$ compõem uma matriz não singular. Se $\alpha$ é conhecida, sempre podemos arrumar os elementos de $\chi_{t}$ de forma tal que a matriz $\alpha$ tenha essa propriedade.

\subsection{Calculando a Representação VAR a partir da Representação VAR Restrita}

o modelo (3.4) pode ser estimado com Mínimos Quadrados Multivariados, método que foi descrito no capítulo 2 . A partir da estimação da expressão (3.4), podemos calcular os parâmetros da Representação VAR, na expressão (3.1). As relações entre o VAR e o VAR restrito, são obtidas facilmente :

$$
\rho=M^{-1} \theta, \quad \rho^{*}=M^{-1} \theta^{*}, \quad \varepsilon_{t}=M^{-1} \eta_{t}, \quad A(L)=M^{-1} B(L) D_{\perp}(L) M
$$

Sendo que a) $\operatorname{det}[B(L)]=0$ tem todas as soluções fora do círculo unitário (porque $\left\{\mathrm{y}_{\mathrm{t}}\right\}$ é um processo estacionário) $\Rightarrow$ $\operatorname{det}[B(1)] \neq 0 \Rightarrow$ posto $[B(1)]=n)$, b) $D_{1}(1)$ tem posto $r$ e c) $M$ tem posto $n$, é claro que $A(1)$ tem posto $r$.

Alternativamente, sendo que $\alpha, \gamma$ conjuntamente com $A^{*}(L)$ foram estimados pelo método de Johansen, estas estimativas podem ser usadas para calcular os coeficientes de B(L), para logo calcular os coefientes de $A(L)$.

\subsection{Exemplo}

Continuamos aqui com o exemplo desenvolvido no capítulo 2, ou seja, um processo VAR(2) não estacionário 3-dimensional, onde os componentes são CI $(1,1)$ :

$$
\left[I_{3}-A_{1} L-A_{2} L^{2}\left\{\begin{array}{l}
\chi_{1 t} \\
\chi_{2 t} \\
\chi_{3 t}
\end{array}\right]=\left[\begin{array}{l}
\rho_{1} \\
\rho_{2} \\
\rho_{3}
\end{array}\right]+\left[\begin{array}{c}
\rho_{1}^{*} \\
\rho_{2}^{*} \\
\rho_{3}^{*}
\end{array}\right] D_{t}+\left[\begin{array}{l}
\varepsilon_{1 t} \\
\varepsilon_{2 t} \\
\varepsilon_{3 t}
\end{array}\right]\right.
$$


com $r=1$, ou seja $k=2$, levando a uma representação MCE :

$$
\left(I_{3}+A_{2} L\right)\left[\begin{array}{c}
\Delta \chi_{1 t} \\
\Delta \chi_{2 t} \\
\Delta \chi_{3 t}
\end{array}\right]=\left[\begin{array}{l}
\rho_{1} \\
\rho_{2} \\
\rho_{3}
\end{array}\right]+\left[\begin{array}{c}
\rho_{1}^{*} \\
\rho_{2}^{*} \\
\rho_{3}^{*}
\end{array}\right] D_{t}-\left[\begin{array}{l}
\gamma_{11} \\
\gamma_{21} \\
\gamma_{31}
\end{array}\right]\left[\begin{array}{lll}
\alpha_{11} & \alpha_{21} & \alpha_{31}
\end{array}\right]\left[\begin{array}{l}
\chi_{1, t-1} \\
\chi_{2, t-1} \\
\chi_{3, t-1}
\end{array}\right]+\left[\begin{array}{l}
\varepsilon_{1 t} \\
\varepsilon_{2 t} \\
\varepsilon_{3 t}
\end{array}\right]
$$

Calcule a matriz $\mathrm{M}$ com o vetor $\alpha$ normalizado, $\mathrm{e} \mathrm{S}_{\mathrm{k}}=\mathrm{I}_{\mathrm{k}}$ :

$$
M=\left[\begin{array}{c}
S_{k} \\
\alpha^{\prime}
\end{array}\right]=\left[\begin{array}{ccc}
1 & 0 & 0 \\
0 & 1 & 0 \\
\alpha_{11}^{*} & \alpha_{21}^{*} & 1
\end{array}\right]
$$

lOBS : esta representação de $M$ requer que a matriz formada pelas últimas $r$ linhas de $\alpha$ seja não singular, no exemplo, de fato é o escalar $\alpha_{31}$ ).

As demais matrizes a calcular, para construir O VAR Restrito, são :

$$
\mathrm{D}(\mathrm{L})=\left[\begin{array}{ccc}
1 & 0 & 0 \\
0 & 1 & 0 \\
0 & 0 & (1-\mathrm{L})
\end{array}\right] \quad \mathrm{D}_{\perp}(\mathrm{L})=\left[\begin{array}{ccc}
(1-\mathrm{L}) & 0 & 0 \\
0 & (1-\mathrm{L}) & 0 \\
0 & 0 & 1
\end{array}\right]
$$

Assim, $y_{t}$ fica :

$$
\mathrm{Y}_{\mathrm{t}}=\left[\begin{array}{ccc}
(1-\mathrm{L}) & 0 & 0 \\
0 & (1-\mathrm{L}) & 0 \\
0 & 0 & 1
\end{array}\right]\left[\begin{array}{ccc}
1 & 0 & 0 \\
0 & 1 & 0 \\
\alpha_{11}^{*} & \alpha_{21}^{*} & 1
\end{array}\right]\left[\begin{array}{l}
\chi_{1 \mathrm{t}} \\
\chi_{2 \mathrm{t}} \\
\chi_{3 \mathrm{t}}
\end{array}\right]=\left[\begin{array}{c}
\Delta \chi_{1 \mathrm{t}} \\
\Delta \chi_{2 \mathrm{t}} \\
\alpha_{11}^{*} \chi_{1 \mathrm{t}}+\alpha_{21}^{*} \chi_{2 \mathrm{t}}+\chi_{3 \mathrm{t}}
\end{array}\right]
$$

onde claramente é observado que $\left\{Y_{t}\right\}$ é um processo estacionário, sendo que cada um dos componentes é estacionário.

0 modelo $\mathrm{B}(\mathrm{L}) \mathrm{y}_{\mathrm{t}}=\theta+\theta^{*} \mathrm{D}_{t}+\eta_{\mathrm{t}}$, onde $\mathrm{Y}_{\mathrm{t}}$ é definido como em $(3.17), \theta, \theta^{*}$ são vetores de 3 -dimensionais de constantes e $\eta_{t}$ é o vetor 3-dimensional de resíduos, é um VAR(2) 
estacionário 3-dimensional, e pode então ser estimado pelo procedimento usual para estimar modelos VAR estacionários. Como já foi comentado, pode ser feito através da aplicação do Método dos Mínimos Quadrados ordinários Multivariado, que de fato se resume a aplicação de Mínimos Quadrados Ordinários a cada uma das $n$ equações separadamente, se a matriz de covariância dos resíduos possui a estrutura apropriada.

As relações entre o VAR e VAR restrito de nosso exemplo ficam, neste caso:

$$
\begin{aligned}
& {\left[\begin{array}{l}
\rho_{1} \\
\rho_{2} \\
\rho_{3}
\end{array}\right]=\left[\begin{array}{ccc}
1 & 0 & 0 \\
0 & 1 & 0 \\
\alpha_{11}^{*} & \alpha_{21}^{*} & 1
\end{array}\right]^{-1}\left[\begin{array}{l}
\theta_{1} \\
\theta_{2} \\
\theta_{3}
\end{array}\right]=\left[\begin{array}{ccc}
1 & 0 & 0 \\
0 & 1 & 0 \\
-\alpha_{11}^{*} & -\alpha_{21}^{*} & 1
\end{array}\right]\left[\begin{array}{c}
\theta_{1} \\
\theta_{2} \\
\theta_{3}
\end{array}\right]=\left[\begin{array}{c}
\theta_{1} \\
\theta_{2} \\
-\alpha_{11}^{*} \theta_{3}-\alpha_{21}^{*} \theta_{2}+\theta_{3}
\end{array}\right]} \\
& {\left[\begin{array}{l}
\rho_{1}^{*} \\
\rho_{2}^{*} \\
\rho_{3}^{*}
\end{array}\right]=\left[\begin{array}{ccc}
1 & 0 & 0 \\
0 & 1 & 0 \\
\alpha_{11}^{*} & \alpha_{21}^{*} & 1
\end{array}\right]^{-1}\left[\begin{array}{l}
\theta_{1}^{*} \\
\theta_{2}^{*} \\
\theta_{3}^{*}
\end{array}\right]=\left[\begin{array}{ccc}
1 & 0 & 0 \\
0 & 1 & 0 \\
-\alpha_{11}^{*} & -\alpha_{21}^{*} & 1
\end{array}\right]\left[\begin{array}{c}
\theta_{1}^{*} \\
\theta_{2}^{*} \\
\theta_{3}^{*}
\end{array}\right]=\left[\begin{array}{c}
\theta_{1}^{*} \\
\theta_{2}^{*} \\
-\alpha_{11}^{*} \theta_{1}^{*}-\alpha_{21}^{*} \theta_{2}^{*}+\theta_{3}^{*}
\end{array}\right]} \\
& {\left[\begin{array}{l}
\varepsilon_{1 t} \\
\varepsilon_{2 t} \\
\varepsilon_{3 t}
\end{array}\right]=\left[\begin{array}{ccc}
1 & 0 & 0 \\
0 & 1 & 0 \\
\alpha_{11}^{*} & \alpha_{21}^{*} & 1
\end{array}\right]^{-1}\left[\begin{array}{c}
\eta_{1 t} \\
\eta_{2 t} \\
\eta_{3 t}
\end{array}\right]=\left[\begin{array}{ccc}
1 & 0 & 0 \\
0 & 1 & 0 \\
-\alpha_{11}^{*} & -\alpha_{21}^{*} & 1
\end{array}\right]\left[\begin{array}{c}
\eta_{1 t} \\
\eta_{2 t} \\
\eta_{3 t}
\end{array}\right]=\left[\begin{array}{c}
\eta_{1 t} \\
\eta_{2 t} \\
-\alpha_{11}^{*} \eta_{3 t}-\alpha_{21}^{*} \eta_{2 t}+\eta_{3 t}
\end{array}\right]}
\end{aligned}
$$

e

$$
\left(I_{3}-A_{1} L-A_{2} L^{2}\right)=\left[\begin{array}{ccc}
1 & 0 & 0 \\
0 & 1 & 0 \\
-\alpha_{11}^{*} & -\alpha_{21}^{*} & 1
\end{array}\right]\left(I_{3}-B_{1} L-B_{2} L^{2}\right)\left[\begin{array}{ccc}
(1-L) & 0 & 0 \\
0 & (1-L) & 0 \\
0 & 0 & 1
\end{array}\right]\left[\begin{array}{ccc}
1 & 0 & 0 \\
0 & 1 & 0 \\
\alpha_{11}^{*} & \alpha_{21}^{*} & 1
\end{array}\right]
$$

onde : 


$$
B_{1}=\left[\begin{array}{lll}
b_{11,} & b_{12,} & b_{13,} \\
b_{21,} & b_{22,} & b_{23,} \\
b_{31,} & b_{32,} & b_{33,}
\end{array}\right] \quad \text { e } \quad B_{2}=\left[\begin{array}{lll}
b_{11,} & b_{12,} & b_{13,22} \\
b_{21,2} & b_{22,} & b_{23,} \\
b_{31,2} & b_{32,} & b_{33,2}
\end{array}\right]
$$




\section{Capítulo 4}

\section{As Representações de Wold e de Tendências Comuns}

Neste capítulo explicaremos como obter a representação de Wold para um processo $\left\{\boldsymbol{x}_{\mathbf{t}}\right\}$ tal que $\boldsymbol{\chi}_{\mathbf{t}} \sim \mathrm{CI}(1,1)$, após transformar as variáveis desse vetor usando o operador diferença. Partindo da representação $\operatorname{VAR}(p)$, e usando as estimativas dos parâmetros e polinomios matriciais nas representações do MCE e do VAR restrito discutidas nos capítulos anteriores, a representação de Wold é facilmente obtida. Estes ítens serão tratados na seção 4.1.

$\mathrm{Na}$ seção 4.2 introduziremos o conceito de representação de Tendências Comuns, e como esta pode ser obtida a partir da representação de Wold.

Na seção 4.3 apresentaremos um exemplo para esclarecer os procedimentos apresentados.

\subsection{A Representação de Wold}

Seja $\left\{\boldsymbol{x}_{\mathbf{t}}\right\}$ um processo $\operatorname{VAR}(\mathrm{p})$ não estacionário :

$$
A(L) \chi_{t}=\rho+\rho^{*} D_{t}+\varepsilon_{t}
$$

co-integrado de ordem $(1,1)$, com $r$ vetores cointegrados, tendo então a representação MCE :

$$
\mathrm{A}^{*}(\mathrm{~L}) \Delta \chi_{t}=\rho+\rho^{*} \mathrm{D}_{\mathrm{t}}-\gamma \alpha^{\prime} \chi_{\mathrm{t}-1}+\varepsilon_{\mathrm{t}}
$$

e a representação VAR restrita :

$$
\mathrm{B}(\mathrm{L}) \mathrm{y}_{\mathrm{t}}=\theta+\theta^{*} \mathrm{D}_{\mathrm{t}}+\eta_{\mathrm{t}}
$$

onde $\chi_{t}$ é um vetor $n \times 1, \rho, \rho^{*}, D_{t}, \varepsilon_{t}, A^{*}(L), \alpha, \gamma, B(L)$, $\boldsymbol{\theta}, \boldsymbol{\theta}^{*}$ e $\eta_{t}$ possuem as especificações feitas em capítulos anteriores.

A expressão (4.2) representa um processo estacionário. Pelo teorema de Wold, todo processo estacionário pode ser 
escrito como a soma de dois processos não correlacionados, um deles determinístico cuja previsão pode ser perfeitamente inferida de seu próprio passado, e o outro tendo uma representação MA de ordem infinita. Como já foi dito no capítulo 2, inverter a expressão (4.2) resulta computacionalmente dificil pois a matriz $A(1)=\gamma \alpha^{\prime}$ é não invertível. No entanto, a representação de Wold é facilmente obtida a partir de (4.1) usando a estimativa de B(L) em (4.3), que por sua vez foi calculada atravês da estimação prévia dos vetores $\alpha$ e $\gamma$ em (4.2) :

1) Substitua o polinômio $A(L)$ em (4.1) pela expressão apresentada em (3.12):

$$
A(L) \chi_{t}=\left[M^{-1} B(L) D_{1}(L) M\right] \chi_{t}=\rho+\rho D_{t}+\varepsilon_{t}
$$

2) Pré-multiplique (4.4) pela expressão $M^{-1} D(L) B(L)^{-1} M$ :

$\left[M^{-1} D(L) B(L)^{-1} M\right]\left[M^{-1} B(L) D_{\perp}(L) M\right] \chi_{t}=\left[M^{-1} D(L) B(L)^{-1} M\right] \rho+\left[M^{-1} D(L) B(L)^{-1} M\right]\left(\varepsilon_{t}+\rho^{*} D_{t}\right)$ obtendo a representação de Wold :

$$
\Delta \chi_{t}=\delta+C(L)\left[\varepsilon_{t}+\rho^{*} D_{t}\right]
$$

onde

$$
C(L)=M^{-1} D(L) B(L)^{-1} M=\sum_{i=0}^{\infty} C_{i} I^{i}, \quad \delta=C(l) \rho
$$

Note que $\mathrm{C}(\mathrm{L})$ é um polinômio matricial absolutamente somável, pois é função de $B(L)^{-1}$, que é absolutamente somável.

A representação de Wold (4.5) permite calcular a chamada função Resposta de Impulsos, que mostra como o vetor $\chi_{t}$ é afetado por choques inesperados em algum/todos os componentes do vetor dos residuos. 


\subsection{A Representação de Tendências Comuns}

A seguir, mostraremos como obter a representação de Tendências Comuns, cujo conceito já foi introduzido no Capítulo 1 .

Substituindo recursivamente em (4.5):

$$
\begin{aligned}
& \chi_{1}=\chi_{0}+\delta+C(L)\left[\varepsilon_{1}+\rho^{*} D_{1}\right] \\
& \chi_{2}=\chi_{1}+\delta+C(L)\left[\varepsilon_{2}+\rho^{*} D_{2}\right]=\chi_{0}+\delta 2+C(L)\left[\varepsilon_{1}+\varepsilon_{2}+\rho^{*} D_{1}+\rho^{*} D_{2}\right]
\end{aligned}
$$

obtemos :

$$
\chi_{t}=\chi_{0}+\delta t+C(L)\left(1+L+L^{2}+\ldots L^{t}\right)\left[\varepsilon_{t}+\rho^{*} D_{t}\right]
$$

Defina $C(L)$ como sendo:

$$
\begin{aligned}
C(L) & =C^{\circ}(\mathrm{L})+C(1) \\
& =\sum_{i=0}^{\infty} C_{i}^{0} L^{i}+\sum_{i=0}^{\infty} C_{i}
\end{aligned}
$$

onde $C^{\circ}(\mathrm{L})=\sum_{i=0}^{\infty} C_{i}{ }^{\circ}{ }^{i}$

Por (4.8), e igualando os coeficientes das potencias em ambos os membros, temos que :

$$
C_{0}=C_{0}^{\circ}+\sum_{i=0}^{\infty} C_{i} \Rightarrow C_{0}^{\circ}=-\sum_{i=1}^{\infty} C_{i}
$$

e para $i>0$, temos que $c_{i}^{\circ}=C_{i}$.

Defina agora $C^{*}\left(L_{)}\right)=\sum_{i=0}^{\infty} C_{i}^{*} L^{i}$ como sendo: 


$$
\begin{aligned}
C^{*}(L)= & C^{0}(L) \sum_{t=0}^{\infty} L^{t} \\
& =-\sum_{i=1}^{\infty} C_{i}+\sum_{i=1}^{\infty} C_{i} L^{i}-\sum_{i=1}^{\infty} C_{i} L+\sum_{i=1}^{\infty} C_{i} L^{i+1} \\
& -\sum_{i=1}^{\infty} C_{i} L^{2}+\sum_{i=1}^{\infty} C_{i} L^{i+2}+\ldots-\sum_{i=1}^{\infty} C_{i} L^{t}+\sum_{i=1}^{\infty} C_{i} L^{i+t}+\ldots
\end{aligned}
$$

Para calcular os coeficientes do polinômio $C^{*}(L)$, igualando os coeficientes das potencias em (4.10), observe que :

$$
\begin{aligned}
& C_{0}^{*}=-\sum_{j=1}^{\infty} C_{j} \\
& C_{1}^{*}=C_{1}-\sum_{j=1}^{\infty} C_{j}=\sum_{j=2}^{\infty} c_{j} \\
& C_{2}^{*}=C_{1}+C_{2}-\sum_{j=1}^{\infty} C_{j}=\sum_{j=3}^{\infty} c_{j} \\
& \cdot \\
& \cdot \\
& C_{i}^{*}=-\sum_{j=1}^{\infty} C_{j}
\end{aligned}
$$

Por $(4.10)$ e (4.11), sendo que $\delta=C(1) \rho$ por (4.6), e para $t$ assumindo valores muito grandes $(t \rightarrow \infty)$, a expressão (4.7) pode ser re-escrita como :

$$
\begin{aligned}
x_{t} & =x_{0}+\delta t+\left[C^{\circ}(L)+C(1)\right] \sum_{i=0}^{t} L^{i}\left(\varepsilon_{t}+\rho^{*} D_{t}\right) \\
& =x_{0}+C(l)\left[\rho t+\sum_{i=0}^{t} L^{i}\left(\varepsilon_{t}+\rho^{*} D_{t}\right)\right]+\left[C^{\circ}(L) \sum_{i=0}^{t} L^{i}\right]\left(\varepsilon_{t}+\rho^{*} D_{t}\right) \\
& =x_{0}+C(l)\left[\rho t+\sum_{i=0}^{t} L^{\dot{1}}\left(\varepsilon_{t}+\rho^{*} D_{t}\right)\right]+C^{*}(L)\left(\varepsilon_{t}+\rho^{*} D_{t}\right)
\end{aligned}
$$




$$
=x_{0}+C(1) \xi_{t}+C^{*}(L)\left(\varepsilon_{t}+\rho^{*} D_{t}\right)
$$

onde :

$$
\xi_{t}=\rho t+\sum_{i=0}^{t} L^{i}\left(\varepsilon_{t}+\rho^{*} D_{t}\right.
$$

o vetor n-dimensional $\xi_{t}$ é um passeio aleatório com desvío ("drift"), $\circ$ que pode ser observado substituindo recursivamente em (4.13), e fazendo a suposição convencional de que $\xi_{\mathbf{s}}=0$ para $s<0$ :

$$
\begin{aligned}
& \xi_{0}=\varepsilon_{0} \\
& \xi_{1}=\rho+\varepsilon_{0}+\varepsilon_{1}+\rho^{*} D_{1}=\rho+\rho^{*} D_{1}+\xi_{0}+\varepsilon_{1} \\
& \xi_{2}=\rho 2+\varepsilon_{0}+\varepsilon_{1}+\varepsilon_{2}+\rho^{*} D_{1}+\rho^{*} D_{2}=\rho+\rho^{*} D_{2}+\xi_{1}+\varepsilon_{2} \\
& \dot{\cdot} \\
& \dot{\xi_{t}}=\rho+\rho^{*} D_{t}+\xi_{t-1}+\varepsilon_{t}
\end{aligned}
$$

o comportamento de longo prazo de $\chi_{t}$ está dominado pelo componente não estacionário $\mathrm{C}(1) \xi_{t}$, (que foi acumulando todos os choques e efeitos das variáveis exógenas no passado). No entanto, lembrando que $z_{t}=\alpha^{\prime} \chi_{t}$, onde $\left\{z_{t}\right\}$ é estacionário e 0 componente $\mathrm{C}(1) \xi_{\mathrm{t}}$ é não estacionário, pré-multiplicando (4.12) por $\alpha^{\prime}$, temos que :

(1) $\alpha^{\prime} C(1)=0$, o que implica que $C(1)$ não é de posto completo (ver apêndice $A$, seção A.1), sendo de posto igual a $\mathrm{n}-\mathrm{r}=\mathrm{k}$. Assim, obtem-se :

$$
z_{\mathrm{t}}=\alpha^{\prime} \chi_{\mathrm{t}}=\alpha^{\prime} \chi_{0}+\alpha^{\prime} \mathrm{C}^{*}(\mathrm{~L}) \varepsilon_{\mathrm{t}}
$$

(2) Adicionalmente, $C^{*}(\mathrm{~L})$ deve ser absolutamente somável, o que deriva da suposição de que $C(L)$ é 1-somável.

Stock \& Watson (1988) mostram como a partir de (4.12) pode ser obtida a representação de tendências comuns. Considere : 
(i) $C(1)$ tem posto $n-r=k<n$ pois $\alpha^{\prime} C(1)=0$, e o posto $(\alpha)=r$. Portanto, existe uma matriz $\mathrm{H}_{1}$, de dimensão $\mathrm{n} \times \mathrm{r}$, e de posto $r$ tal que $\mathrm{C}(1) \mathrm{H}_{1}=0$.

(ii) Se $\mathrm{H}_{2}$ é uma matriz de dimensão $n \times k$, com posto $k$, com colunas ortogonais às colunas de $\mathrm{H}_{1}$, então $\mathrm{K}=: \mathrm{C}(1) \mathrm{H}_{2}$ tem posto $\mathrm{k}$ (note que $\mathrm{C}(1)^{\prime}$ e $\mathrm{H}_{2}$ estão no mesmo subespaço).

(iii) A matriz $\mathrm{H}=\left(\mathrm{H}_{1} \mathrm{H}_{2}\right)$ de dimensão $\mathrm{n} \times \mathrm{n}$ é não singular, e $\mathrm{C}(1) \mathrm{H}=(0 \mathrm{~K})=\mathrm{KS}_{\mathrm{k}}$, onde $\mathrm{S}_{\mathrm{k}}$ é a matriz de seleção de dimensão $k \times n$. Ou seja $S_{k}=\left[O_{k x(n-k)} I_{k}\right]$, onde $O_{k x(n-k)}$ é uma matriz de zeros de dimensão $k \times(n-k)$. (iii))

(ver apéndice A, seção A.1, para justificar (i), (ii) e

Então, partindo de (4.12) e por (iii), obtemos :

$$
\begin{aligned}
\chi_{t} & =\chi_{0}+C(1) H^{-1} \xi_{t}+C^{*}(L)\left[\varepsilon_{t}+\rho^{*} D_{t}\right] \\
& =\chi_{0}+K S_{k} H^{-1} \xi_{t}+C^{*}(L)\left[\varepsilon_{t}+\rho^{*} D_{t}\right] \\
& =\chi_{0}+K \tau_{t}+\Phi(L)\left[v_{t}+\Psi D_{t}\right]
\end{aligned}
$$

sendo que :

$$
\tau_{t}=\mu+\mu^{\star} D_{t}+\tau_{t-1}+\varphi_{t}
$$

onde

$$
\begin{gathered}
\tau_{\mathrm{t}}=\mathrm{S}_{\mathrm{k}} \mathrm{H}^{-1} \xi_{\mathrm{t}}, \Phi(\mathrm{L})=\mathrm{C}^{*}(\mathrm{~L}) \mathrm{F}^{-1}, \quad v_{\mathrm{t}}=\mathrm{F} \varepsilon_{\mathrm{t}}, \\
\Psi_{\mathrm{t}}=\mathrm{F} \rho^{*}, \quad \mathrm{~F}:=\left[\begin{array}{ll}
\mathrm{F}_{\mathrm{k}}^{\prime} & \left.\mathrm{F}_{\mathrm{r}}^{\prime}\right]
\end{array}\right.
\end{gathered}
$$

Por (4.14), temos que $\mu=\mathrm{S}_{\mathrm{k}} \mathrm{H}^{-1} \rho, \mu^{*}=\mathrm{S}_{\mathrm{k}} \mathrm{H}^{-1} \boldsymbol{\rho}^{*}$, e $\boldsymbol{\varphi}_{\mathrm{t}}=\mathrm{S}_{\mathrm{k}} \mathrm{H}^{-1} \boldsymbol{\varepsilon}_{\mathrm{t}}$. Note que $\tau_{t}$ é um vetor $k \times 1$, tendo tantos elementos como tendências são observadas, o fato de $\tau_{t}$ ser $k$-dimensional deriva do fato do modelo ser co-integrado de ordem $r=n-k$. A representação de Tendências Comuns expressa o vetor $\chi_{t}$ como uma combinação linear de $k$ passeios aleatórios com drift $\mu+\mu^{*} D_{t}$. 


\subsection{Exemplo}

Prosseguindo com nosso exemplo desenvolvido nos capítulos anteriores, ou seja, um processo 3-dimensional não estacionário $A R(2)$, co-integrado com $r=1$, implicando $k=2$ (número de tendências) e variável dummy $D_{t}$ 1-dimensional, a representação de Wold deste processo fica :

$$
\left[\begin{array}{l}
\Delta x_{1 \mathrm{t}} \\
\Delta \chi_{2 t} \\
\Delta \chi_{3 t}
\end{array}\right]=\left[\begin{array}{l}
\delta_{1} \\
\delta_{2} \\
\delta_{3}
\end{array}\right]+C(\mathrm{~L})\left\{\left[\begin{array}{l}
\varepsilon_{1 t} \\
\varepsilon_{2 t} \\
\varepsilon_{3 t}
\end{array}\right]+\left[\begin{array}{l}
\rho_{1}^{*} \\
\rho_{2}^{*} \\
\rho_{3}^{*}
\end{array}\right]\right\}
$$

onde $C(L)$ pode ser calculado a partir do conhecimento dos parâmetros na representação de MCE e/ou da estimativa do polinômio matricial $B(L)$ na representação VAR Restrita, de acordo com a expressão (4.6).

A representação de Tendências Comuns para este processo fica :

$$
\left.\left.\left[\begin{array}{l}
\chi_{1 \mathrm{t}} \\
\chi_{2 \mathrm{t}} \\
\chi_{3 \mathrm{t}}
\end{array}\right]=\left[\begin{array}{l}
\chi_{10} \\
\chi_{20} \\
\chi_{30}
\end{array}\right]+\left[\begin{array}{ll}
\kappa_{11} & \kappa_{12} \\
\kappa_{21} & \kappa_{22} \\
\kappa_{31} & \kappa_{32}
\end{array}\right]\left[\begin{array}{l}
\tau_{1 \mathrm{t}} \\
\tau_{2 \mathrm{t}}
\end{array}\right]+\Phi(\mathrm{L})\right)\left[\begin{array}{l}
v_{1 \mathrm{t}} \\
v_{2 \mathrm{t}} \\
v_{3 \mathrm{t}}
\end{array}\right]+\left[\begin{array}{l}
\Psi_{1} \\
\Psi_{1} \\
\Psi_{1}
\end{array}\right]\right]
$$

onde $\Phi(L)$ é um polinômio matricial 3-dimensional absolutamente somável e :

$$
\left[\begin{array}{l}
\tau_{1 t} \\
\tau_{2 t}
\end{array}\right]=\left[\begin{array}{l}
\mu_{1} \\
\mu_{2}
\end{array}\right]+\left[\begin{array}{l}
\mu_{1}^{*} \\
\mu_{2}^{*}
\end{array}\right] D_{t}+\left[\begin{array}{l}
\tau_{1, t-1} \\
\tau_{2, t-1}
\end{array}\right]+\left[\begin{array}{l}
\varphi_{1 t} \\
\varphi_{2 t}
\end{array}\right]
$$




\title{
Capítulo 5 \\ Identificação dos Parâmetros da Representação de Tendências Comuns
}

\begin{abstract}
Neste capítulo, na seção 5.1, mostraremos como identificar os parâmetros da representação de Tendências Comuns, partindo do conhecimento dos parâmetros das Representações discutidas nos capítulos anteriores. Adicionalmente, na seção 5.2, será apresentado um exemplo para esclarecer o procedimento.
\end{abstract}

\subsection{Procedimento de Identificação}

Repetimos a seguir as especificações da representação de Tendências Comuns discutida na introdução e no capítulo 4, com a finalidade de deixar claro quais são os supostos usados que justificarão o procedimento de identificação dos parâmetros.

Seja um processo $\left\{\chi_{t}\right\}$ n-dimensional, co-integrado $(1,1)$ com $r$ vetores co-integrados, implicando que o número de tendências é igual a $k=n-r$ e cuja representação de Tendências Comuns está dado por :

$$
\begin{gathered}
\chi_{t}=\chi_{0}+K \tau_{t}+\Phi(L)\left[v_{t}+\Psi D_{t}\right], \\
\tau_{t}=\mu+\mu^{*} D_{t}+\tau_{t-1}+\varphi_{t}
\end{gathered}
$$

onde :

(i) $x_{0}$ é o vetor constante contendo os valores iniciais de $\boldsymbol{x}_{t}$.

(ii) Os componentes não estacionários (permanentes) e estacionários (transitórios) de $\chi_{t}$ estão capturados por $\mathrm{K} \tau_{t} \mathrm{e}$ $\Phi(\mathrm{L}) \boldsymbol{v}_{\mathrm{t}}$ respectivamente.

(iii) K é uma matriz de constantes de dimensão $n \times k$, onde $\mathrm{k}$ representa $\mathrm{o}$ numero de tendências. $\tau_{\mathrm{t}}$ é um vetor de dimensão $k \times 1$, a variável de tendência. A sequência n-dimensional $\left\{\boldsymbol{v}_{\mathbf{t}}\right\}$ é um ruido branco com $E\left(\boldsymbol{v}_{\mathbf{t}}\right)=0$ e 
$E\left(\boldsymbol{v}_{\mathbf{t}} \boldsymbol{v}_{\mathbf{t}}\right)=I_{n}$ a $n \times n$ matriz identidade. Assumimos que $\boldsymbol{v}_{\mathbf{s}}=0$ para todo $s \leq 0$, e que $\Phi(L)$ é um polinomio absolutamente somável. Dt é um vetor d-dimensional de variáveis dummy.

(iv) $\mu$ e $\mu^{*}$ são vetores $k$-dimensionais de constantes. $\left\{\varphi_{t}\right\}$ é uma sequência ruido branco $\operatorname{com} E\left(\varphi_{t}\right)=0$ e $E\left(\varphi_{t} \varphi_{t}^{\prime}\right)=I_{k}$

Para calcular os parâmetros do modelo (5.1)-(5.2) proceda da seguinte maneira :

(1) Considere as restrições derivadas da existência de co-integração. Para que o processo $\left\{z_{t}\right\}$ onde $z_{t}=\alpha^{\prime} \chi_{t}$ seja estacionário, sendo que $\tau_{\mathrm{t}}$ é não estacionário, os $n k$ componentes da matriz $\mathrm{K}$ na expressão (5.1) devem satisfazer as seguintes restrições :

$$
\alpha^{\prime} \mathrm{K}=0
$$

Dado $\boldsymbol{\alpha}$, as restrições de co-integração fornecem então $r k=(n-k) k$ equações que podem ser usadas para determinar. alguns dos $n k$ componentes de $K$.

(2) Após ter obtido rk restrições em (1), precisamos de nk-rk restrições adicionais para obter os restantes coeficientes de $\mathrm{K}$.

Substituindo (5.2) em (5.1) temos que :

$$
\begin{aligned}
\chi_{t} & =\chi_{0}+K\left(\mu+\mu^{*} D_{t}+\tau_{t-1}+\varphi_{t}\right)+\Phi(L)\left[v_{t}+\Psi D_{t}\right] \\
& =\chi_{0}+K \mu+K \mu^{*} D_{t}+K \tau_{t-1}+K \varphi_{t}+\Phi(L)\left[v_{t}+\Psi D_{t}\right]
\end{aligned}
$$

Por outro lado, substituindo (4.14) em (4.12) e lembrando que $\delta=C(1) \rho$, temos que :

$$
\begin{aligned}
\chi_{t} & =\chi_{0}+C(1)\left(\rho+\rho^{*} D_{t}+\xi_{t-1}+\varepsilon_{t}\right)+C^{*}(L)\left[\varepsilon_{t}+\rho^{*} D_{t}\right] \\
& =\chi_{0}+C(l) \rho+C(l) \rho^{*} D_{t}+C(l) \xi_{t-1}+C(l) \varepsilon_{t}+C^{*}(L)\left[\varepsilon_{t}+\rho^{*} D_{t}\right] \\
& =\chi_{0}+\delta+C(l) \rho^{*} D_{t}+C(l) \xi_{t-1}+C(l) \varepsilon_{t}+C^{*}(L)\left[\varepsilon_{t}+\rho^{*} D_{t}\right]
\end{aligned}
$$

Comparando as expressões (5.4) e (5.5) temos que : 


$$
\delta=K \mu, \quad C(1) \rho^{*}=K \mu^{*} \text { e } C(1) \varepsilon_{t}=K \varphi_{t} \text { para todo } t
$$

As igualdades (5.6) na verdade já foram mostradas em (4.18) quando derivamos a representação de Tendências Comuns a partir da representação de Wold. que :

Lembrando que $E\left[\varepsilon_{t} \varepsilon_{t}^{\prime}\right]=\Sigma$ e $E\left[\varphi_{t} \varphi_{t}^{\prime}\right]=I_{k}$, e por (5.6), segue

$$
\begin{aligned}
& \mathrm{E}\left(\mathrm{K} \varphi_{\mathrm{t}} \varphi_{\mathrm{t}}^{\prime} \mathrm{K}^{\prime}\right)=\mathrm{E}\left(\mathrm{C}(\mathrm{l}) \varepsilon_{\mathrm{t}} \varepsilon_{\mathrm{t}}^{\prime} \mathrm{C}(\mathrm{l})^{\prime}\right) \\
& \mathrm{KE}\left(\varphi_{\mathrm{t}} \varphi_{\mathrm{Z}}^{\prime} \mathrm{K}^{\prime}=\mathrm{C}(\mathrm{l}) \mathrm{E}\left(\varepsilon_{\mathrm{t}} \varepsilon_{\mathrm{U}}^{\prime} \mathrm{C}(\mathrm{l})^{\prime}\right.\right. \\
& \mathrm{KK}^{\prime}=\mathrm{C}(\mathrm{l}) \sum \mathrm{C}\left(\mathrm{l}^{\prime}\right.
\end{aligned}
$$

Dado $C(1)$, que é de posto $k$, e $\sum$, a equação (5.7) fornece $k(k+1) / 2$ restrições sobre $K$, sendo que o segundo membro de (5.7) é uma matriz simétrica.

Lembre que, por (4.6), podemos obter uma estimativa de C(1), a partir das estimativas dos parâmetros nas representações de MCE e de VAR restrito :

$$
C(l)=M^{-1} D(l) B(l)^{-1} M
$$

onde $\mathrm{C}(1)$ é de dimensão $\mathrm{n} \times \mathrm{n}$. Uma estimativa de $\sum$ é obtida a partir da matriz de covariância estimada no VAR restrito, usando (3.12).

(3) Para finalizarmos a identificação de todos os componentes de $\mathrm{K}$, precisamos impor $n k-(n-k) k-k(k+1) / 2=k(k-$ 1) $/ 2$ restrições adicionais, que devem ser escolhidas pelo analista com base em critérios adequados.

(4) Alternativamente, para a obtenção dos elementos de K, King et alli. $(1987,1991)$ propõe escrever a matriz K como $\mathrm{K}_{0} \pi$ onde $\mathrm{K}_{0}$ é uma matriz $\mathrm{n} \times \mathrm{k}$ de posto $\mathrm{k}$, com seus elementos escolhidos de tal forma que $\alpha^{\prime} K_{0}=0$, e onde os parâmetros livres de $\mathrm{K}$ constituem uma matriz $\pi$ de dimensão $\mathrm{k} \times \mathrm{k}$. Substituindo $\mathrm{K}_{0} \pi$ em (5.7), pre-multiplicando por $\left[\mathrm{K}_{0}^{\prime} \mathrm{K}_{0}\right]^{-1} \mathrm{~K}_{0}{ }^{\prime}$ e pos-multiplicando por $\mathrm{K}_{0}\left[\mathrm{~K}_{0}{ }_{0} \mathrm{~K}_{0}\right]^{-1}$, temos que :

$$
\begin{aligned}
& \mathrm{K}_{0} \pi \pi^{\prime} \mathrm{K}_{0}^{\prime}=\mathrm{C}(\mathrm{l}) \Sigma \mathrm{C}(\mathrm{l})^{\prime} \\
& {\left[\mathrm{K}_{0}^{\prime} \mathrm{K}_{0}\right]^{-1} \mathrm{~K}_{0}^{\prime} \mathrm{K}_{0} \pi \pi^{\prime} \mathrm{K}_{0}^{\prime} \mathrm{K}_{0}\left[\mathrm{~K}_{0}^{\prime} \mathrm{K}_{0}\right]^{-1}=\left[\mathrm{K}_{0}^{\prime} \mathrm{K}_{0}\right]^{-1} \mathrm{~K}_{0}^{\prime} \mathrm{C}(\mathrm{l}) \sum \mathrm{C}(1)^{\prime} \mathrm{K}_{0}\left[\mathrm{~K}_{0}^{\prime} \mathrm{K}_{0}\right]^{-1}} \\
& \pi \pi^{\prime}=\left[\mathrm{K}_{0}^{\prime} \mathrm{K}_{0}\right]^{-1} \mathrm{~K}_{0}^{\prime} \mathrm{C}(\mathrm{l}) \sum \mathrm{C}(1)^{\prime} \mathrm{K}_{0}\left[\mathrm{~K}_{0}^{\prime} \mathrm{K}_{0}\right]^{-1}
\end{aligned}
$$


O lado direito de (5.9) é uma matriz kxk positiva definida, com $k(k+1) / 2$ únicos parâmetros. Em (5.9), podemos determinar $k(k+1) / 2$ parâmetros de $\pi$, fazendo uma decomposição de Choleski (ver apéndice $A$, seção A.3) de $\pi \pi^{\prime}$, implicando uma matriz $\pi$ triangular inferior. Dada esta estimativa de $\pi$, podemos calcular então $\mathrm{K}$ :

$$
\mathrm{K}=\mathrm{K}_{0} \pi
$$

(5) Para o cálculo dos outros parâmetros da representação de Tendências Comuns, por $(5.6)$ e (3.12) temos que :

$$
\begin{aligned}
& \mathrm{K} \mu=\delta=\mathrm{C}(\mathrm{l}) \rho=\mathrm{C}(\mathrm{l}) \mathrm{M}^{-1} \theta \\
& \mu=\left(\mathrm{K}^{\prime} \mathrm{K}\right)^{-1} \mathrm{~K}^{\prime} \mathrm{C}(\mathrm{l}) \mathrm{M}^{-1} \theta \\
& \mathrm{K} \mu^{*}=\mathrm{C}(\mathrm{l}) \rho^{*}=\mathrm{C}(\mathrm{l}) \mathrm{M}^{-1} \theta^{*} \\
& \mu^{*}=\left(\mathrm{K}^{\prime} \mathrm{K}\right)^{-1} \mathrm{KC}(\mathrm{l}) \mathrm{M}^{-1} \theta^{*}
\end{aligned}
$$

\subsection{Exemplo}

Prosseguimos com o exemplo desenvolvido em capítulos anteriores, ou seja um processo não estacionário 3dimensional, co-integrado com $r=1$, implicando $k=2$, cuja representação em Tendências Comuns, como já vimos, é dado por :

$$
\begin{aligned}
& {\left[\begin{array}{l}
x_{1 t} \\
x_{2 t} \\
\chi_{3 t}
\end{array}\right]=\left[\begin{array}{l}
\chi_{10} \\
\chi_{20} \\
\chi_{30}
\end{array}\right]+\left[\begin{array}{ll}
\kappa_{11} & \kappa_{12} \\
\kappa_{21} & \kappa_{22} \\
\kappa_{31} & \kappa_{32}
\end{array}\right]\left[\begin{array}{l}
\tau_{1 t} \\
\tau_{2 t}
\end{array}\right]+\Phi(L)\left[\left[\begin{array}{l}
v_{1 t} \\
v_{2 t} \\
v_{3 t}
\end{array}\right]+\left[\begin{array}{l}
\Psi_{1} \\
\Psi_{1} \\
\Psi_{1}
\end{array}\right] D_{t}\right],} \\
& {\left[\begin{array}{l}
\tau_{1 t} \\
\tau_{2 t}
\end{array}\right]=\left[\begin{array}{l}
\mu_{1} \\
\mu_{2}
\end{array}\right]+\left[\begin{array}{l}
\mu_{1}^{*} \\
\mu_{2}^{*}
\end{array}\right] D_{t}+\left[\begin{array}{l}
\tau_{1, t-1} \\
\tau_{2, t-1}
\end{array}\right]+\left[\begin{array}{l}
\varphi_{1 t} \\
\varphi_{2 t}
\end{array}\right]}
\end{aligned}
$$

A seguir, mostramos como identificar os parâmetros deste processo, seguindo os procedimentos (1), (2), (3) e (4) descritos na seção anterior.

(1) Pela restrição (5.3) temos : 


$$
\left[\begin{array}{lll}
\alpha_{11}^{*} & \alpha_{21}^{*} & 1
\end{array}\right]\left[\begin{array}{ll}
\kappa_{11} & \kappa_{12} \\
\kappa_{21} & \kappa_{22} \\
\kappa_{31} & \kappa_{32}
\end{array}\right]=\left[\begin{array}{l}
\alpha_{11}^{*} \kappa_{11}+\alpha_{21}^{*} \kappa_{21}+\kappa_{31} \\
\alpha_{11}^{*} \kappa_{12}+\alpha_{21}^{*} \kappa_{22}+\kappa_{32}
\end{array}\right]^{\prime}=\left[\begin{array}{l}
0 \\
0
\end{array}\right]^{\prime}
$$

Em (5.14) foram obtidas duas $(r k=1 \times 2=2)$ equações para determinar $6(n k=3 \times 2)$ incógnitas $\left(\kappa_{i j} i=1,2,3, j=1,2\right)$.

(2) Calcule agora, o segundo membro na expressão (5.7) e defina :

$$
C(1) \sum C(l)^{\prime}=\left[\begin{array}{lll}
r_{11} & r_{12} & r_{13} \\
r_{21} & r_{22} & r_{23} \\
r_{31} & r_{32} & r_{33}
\end{array}\right]
$$

de dimensão $3 \times 3$, simétrica, e de posto 2 , pois $k=2$. A expressão (5.15) fornece $2(2+1) / 2=3$ restrições sobre $K$.

Logo, por (5.7), temos que:

$$
\mathrm{KK}^{\prime}=\left[\begin{array}{ccc}
\kappa_{11}^{2}+\kappa_{12}^{2} & \kappa_{11} \kappa_{21}+\kappa_{12} \kappa_{22} & \kappa_{11} \kappa_{31}+\kappa_{12} \kappa_{32} \\
\kappa_{21} \kappa_{11}+\kappa_{22} \kappa_{12} & \kappa_{21}^{2}+\kappa_{22}^{2} & \kappa_{21} \kappa_{31}+\kappa_{22} \kappa_{32} \\
\kappa_{31} \kappa_{11}+\kappa_{32} \kappa_{12} & \kappa_{31} \kappa_{21}+\kappa_{32} \kappa_{22} & \kappa_{31}^{2}+\kappa_{32}^{2}
\end{array}\right]=\left[\begin{array}{ccc}
r_{11} & r_{12} & r_{13} \\
r_{21} & r_{22} & r_{23} \\
r_{31} & r_{32} & r_{33}
\end{array}\right]
$$

Sendo que a matriz definida em (5.15) é de posto 2, por operações elementares de matrizes, em (5.16), temos:

$$
\left[\begin{array}{ccc}
\kappa_{11}^{2}+\kappa_{12}^{2} & \kappa_{11} \kappa_{21}+\kappa_{12} \kappa_{22} & 0 \\
\kappa_{21} \kappa_{11}+\kappa_{22} \kappa_{12} & \kappa_{21}^{2}+\kappa_{22}^{2} & 0 \\
0 & 0 & 0
\end{array}\right]=\left[\begin{array}{ccc}
r_{11} & r_{12} & 0 \\
r_{21} & r_{22} & 0 \\
0 & 0 & 0
\end{array}\right]
$$

Assim, por (5.14) e (5.17), ficamos com o seguinte sistema de equações, para calcular os elementos da matriz $\mathrm{K}$ : 


$$
\begin{aligned}
& \alpha_{11}^{*} \kappa_{11}+\alpha_{21}^{*} \kappa_{21}+\kappa_{31}=0 \\
& \alpha_{11}^{*} \kappa_{12}+\alpha_{21}^{*} \kappa_{22}+\kappa_{32}=0 \\
& \kappa_{11}^{2}+\kappa_{12}^{2}=r_{11} \\
& \kappa_{11} \kappa_{21}+\kappa_{12} \kappa_{22}=r_{12} \\
& \kappa_{21}^{2}+\kappa_{22}^{2}=r_{22}
\end{aligned}
$$

(3) No nosso exemplo, foi possível determinar $(\mathrm{n}-\mathrm{k}) \mathrm{k}+\mathrm{k}(\mathrm{k}+1) / 2=5$ restrições para determinar os $\mathrm{nk}=6$ componentes da matriz $K$. Faltam determinar então $\mathrm{nk}-(\mathrm{n}-\mathrm{k}) \mathrm{k}+\mathrm{k}(\mathrm{k}+1) / 2=\mathrm{k}(\mathrm{k}-12) / 2=1$ restrição, para que o sistema seja exatamente identificado.

(4) Alternativamente a (1), (2) e (3), podemos usar o seguinte procedimento : Escolha os componentes de uma matriz $\mathrm{K}_{0}$ de modo que $\alpha^{\prime} \mathrm{K}_{0}=0$ onde $\mathrm{K}_{0}$ é de dimensão $3 \times 2$. É necessário escolher 4 componentes, sendo que os outros dois são determinados pelo sistema :

$$
\alpha^{\prime} \mathrm{K}_{0}=\left[\begin{array}{lll}
\alpha_{11}^{*} & \alpha_{21}^{*} & 1
\end{array}\right]\left[\begin{array}{ll}
\kappa_{11 \rho} & \kappa_{12 \rho} \\
\kappa_{21, \rho} & \kappa_{22 \rho} \\
\kappa_{31, \rho} & \kappa_{32 \rho}
\end{array}\right]=\left[\begin{array}{l}
\alpha_{11}^{*} \kappa_{11 \rho}+\alpha_{21}^{*} \kappa_{21, \rho}+\kappa_{31 \rho} \\
\alpha^{*}{ }_{11} \kappa_{12 \rho}+\alpha_{21}^{*} \kappa_{22 \rho}+\kappa_{32 \rho}
\end{array}\right]^{\prime}=\left[\begin{array}{l}
0 \\
0
\end{array}\right]^{\prime}
$$

Para determinar os valores de $K_{o}$, tome cuidado de que a escolha de componentes derive na constituição de uma matriz $K_{\circ}$ com vetores linearmente independentes. Escolha, por exemplo :

$$
\kappa_{11,0}=\kappa_{31,0}=\kappa_{32,0}=1, \kappa_{12,0}=0
$$

Assim, o sistema de equações em (5.19) fica :

$$
\begin{aligned}
& \alpha_{11}^{*}+\alpha_{21}^{*} \kappa_{21 \rho}+1=0 \\
& \alpha_{21}^{*} \kappa_{22 \rho}+1=0
\end{aligned} \quad \Rightarrow \quad \begin{aligned}
& \kappa_{21 \rho}=-\frac{\left(1+\alpha_{11}^{*}\right)}{\alpha_{21}^{*}} \\
& \kappa_{22 \rho}=-\frac{1}{\alpha_{21}^{*}}
\end{aligned}
$$

A matriz $\mathrm{K}_{0}$ fica : 


$$
K_{0}=\left[\begin{array}{cc}
1 & 0 \\
-\frac{\left(1+\alpha_{11}^{*}\right)}{\alpha_{21}^{*}} & -\frac{1}{\alpha_{21}^{*}} \\
1 & 1
\end{array}\right]
$$

Calcule e defina $\left(\mathrm{K}_{0}{ }^{\prime} \mathrm{K}_{0}\right)^{-1} \mathrm{~K}_{0}{ }^{\prime}$ :

$$
\left[\left[\begin{array}{ccc}
1 & -\frac{\left(1+\alpha_{11}^{*}\right)}{\alpha_{21}^{*}} & 1 \\
0 & -\frac{1}{\alpha_{21}^{*}} & 1
\end{array}\right]\left[\begin{array}{cc}
1 & 0 \\
-\frac{\left(1+\alpha_{11}^{*}\right)}{\alpha_{21}^{*}} & -\frac{1}{\alpha_{21}^{*}} \\
1 & 1
\end{array}\right]\right]^{-1}\left[\begin{array}{ccc}
1 & -\frac{\left(1+\alpha_{11}^{*}\right)}{\alpha_{21}^{*}} & 1 \\
0 & -\frac{1}{\alpha_{21}^{*}} & 1
\end{array}\right]=\left[\begin{array}{lll}
k_{11 \rho} & k_{12 \rho} & k_{13 \rho} \\
k_{21 \rho} & k_{22 \rho} & k_{23 \rho}
\end{array}\right]
$$

Assim podemos escrever, de acordo com a expressão (5.9) :

$$
\left[\begin{array}{ll}
\pi_{11} & \pi_{12} \\
\pi_{21} & \pi_{22}
\end{array}\right]\left[\begin{array}{ll}
\pi_{11} & \pi_{12} \\
\pi_{21} & \pi_{22}
\end{array}\right]^{\prime}=\left[\begin{array}{lll}
\mathrm{k}_{11 \rho} & \mathrm{k}_{12 \rho} & \mathrm{k}_{13 \rho} \\
\mathrm{k}_{21 \rho} & \mathrm{k}_{22 \rho} & \mathrm{k}_{23 \rho}
\end{array}\right]\left[\begin{array}{lll}
r_{11} & \mathrm{r}_{12} & \mathrm{r}_{13} \\
r_{21} & \mathrm{r}_{22} & \mathrm{r}_{23} \\
r_{31} & r_{32} & \mathrm{r}_{33}
\end{array}\right]\left[\begin{array}{ll}
\mathrm{k}_{11 \rho} & \mathrm{k}_{21 \rho} \\
\mathrm{k}_{12 \rho} & \mathrm{k}_{22 \rho} \\
\mathrm{k}_{13 \rho} & \mathrm{k}_{23 \rho}
\end{array}\right]
$$

e calcular os componentes da matriz $\pi$ de acordo a uma decomposição de Choleski da matriz que é calculada no segundo membro de (5.22), sendo que é uma matriz positiva definida. Por fim, podemos calcular K :

$$
\left[\begin{array}{ll}
\kappa_{11} & \kappa_{12} \\
\kappa_{21} & \kappa_{22} \\
\kappa_{31} & \kappa_{32}
\end{array}\right]=\left[\begin{array}{ll}
\kappa_{11 \rho} & \kappa_{12 \rho} \\
\kappa_{21 \rho} & \kappa_{22 \rho} \\
\kappa_{31 \rho} & \kappa_{32 \rho}
\end{array}\right]\left[\begin{array}{ll}
\pi_{11} & \pi_{12} \\
\pi_{21} & \pi_{22}
\end{array}\right]
$$

(5) Procedemos agora ao cálculo do resto dos parâmetros da representação de Tendências Comuns de nosso exemplo. Para facilitar a notação calcule e defina : 


$$
\begin{gathered}
{\left[\left[\begin{array}{ll}
\kappa_{11} & \kappa_{12} \\
\kappa_{21} & \kappa_{22} \\
\kappa_{31} & \kappa_{32}
\end{array}\right]\left[\begin{array}{ll}
\kappa_{11} & \kappa_{12} \\
\kappa_{21} & \kappa_{22} \\
\kappa_{31} & \kappa_{32}
\end{array}\right]\right]^{-1}\left[\begin{array}{ll}
\kappa_{11} & \kappa_{12} \\
\kappa_{21} & \kappa_{22} \\
\kappa_{31} & \kappa_{32}
\end{array}\right]^{\prime}=\left[\begin{array}{lll}
k_{11} & k_{12} & k_{13} \\
k_{21} & k_{22} & k_{23}
\end{array}\right]} \\
C(1)=\left[\begin{array}{lll}
c_{11} & c_{12} & c_{13} \\
c_{21} & c_{22} & c_{23} \\
c_{31} & c_{32} & c_{33}
\end{array}\right]
\end{gathered}
$$

Lembrando que :

$$
\mathbf{M}^{-1}=\left[\begin{array}{ccc}
1 & 0 & 0 \\
0 & 1 & 0 \\
-\alpha_{11}^{*} & -\alpha_{21}^{*} & 1
\end{array}\right]
$$

calcule os outros parâmetros da representação de Tendências Comuns :

$$
\begin{gathered}
{\left[\begin{array}{l}
\mu_{1} \\
\mu_{2}
\end{array}\right]=\left[\begin{array}{lll}
k_{11} & k_{12} & k_{13} \\
k_{21} & k_{22} & k_{23}
\end{array}\right]\left[\begin{array}{lll}
c_{11} & c_{12} & c_{13} \\
c_{21} & c_{22} & c_{23} \\
c_{31} & c_{32} & c_{33}
\end{array}\right]\left[\begin{array}{ccc}
1 & 0 & 0 \\
0 & 1 & 0 \\
-\alpha_{11}^{*} & -\alpha_{21}^{*} & 1
\end{array}\right]\left[\begin{array}{l}
\theta_{1} \\
\theta_{2} \\
\theta_{3}
\end{array}\right]} \\
{\left[\begin{array}{l}
\mu_{1}^{*} \\
\mu_{2}^{*}
\end{array}\right]=\left[\begin{array}{lll}
k_{11} & k_{12} & k_{13} \\
k_{21} & k_{22} & k_{23}
\end{array}\right]\left[\begin{array}{lll}
c_{11} & c_{12} & c_{13} \\
c_{21} & c_{22} & c_{23} \\
c_{31} & c_{32} & c_{33}
\end{array}\right]\left[\begin{array}{ccc}
1 & 0 & 0 \\
0 & 1 & 0 \\
-\alpha_{11}^{*} & -\alpha_{21}^{*} & 1
\end{array}\right]\left[\begin{array}{l}
\theta_{1}^{*} \\
\theta_{2}^{*} \\
\theta_{3}^{*}
\end{array}\right]}
\end{gathered}
$$


Capítulo 6

\section{Cálculo da Função Resposta de Impulso Associada à Representação de Tendências Comuns}

Com frequência estamos interessados no estudo de choques nas variáveis de um modelo. A chamada "função resposta de impulsos" é simplesmente uma tabela com os coeficientes da representação MA, dando a resposta da variável no sistema a um impulso não esperado de algum componente do vetor dos resíduos.

Neste capítulo, na seção 6.1 veremos como calcular a função resposta de impulsos associada à representação de Tendências Comuns. Em outras palavras, veremos como as variáveis do sistema são afetadas a partir de choques nos vetores dos resíduos naquela representação. Faremos isto usando o cálculo de parâmetros já feito em outras representações, porém deveremos impor restrições adicionais. Como vem sendo feito, após a explicação do procedimento, continuar-se-á com o desenvolvimento do exemplo apresentado em capítulos anteriores, na seção 6.2 .

\subsection{Procedimento de Identificação}

Seja um processo $\left\{\chi_{t}\right\}$ n-dimensional não estacionário, cointegrado $(1,1)$ com $r$ vetores co-integrados, implicando que o número de tendências é igual k=n-r, cuja representação de Wold está dada por :

$$
\Delta \chi_{t}=\delta+C(L)\left[\varepsilon_{t}+\rho{ }^{*} D_{t}\right]
$$

e a representação de Tendências Comuns é :

$$
\begin{gathered}
\chi_{t}=\chi_{0}+K \tau_{t}+\Phi(L)\left[v_{t}+\Psi D_{t}\right] \\
\tau_{t}=\mu+\mu^{*} D_{t}+\tau_{t-1}+\varphi_{t}
\end{gathered}
$$

onde as especificações dos parâmetros, variáveis e processos são idénticas às definidas em capítulos anteriores. 
Para o cálculo da função de resposta de impulsos associada á representação de tendências comuns, temos que impor restrições adicionais ao modelo. Defina :

$$
\begin{gathered}
v_{t}=\left[\begin{array}{l}
\varphi_{t} \\
\psi_{t}
\end{array}\right]=F \varepsilon_{t} \quad F_{:}=\left[\begin{array}{l}
F_{k} \\
F_{r}
\end{array}\right] \quad \therefore\left[\begin{array}{l}
\varphi_{t} \\
\psi_{t}
\end{array}\right]=\left[\begin{array}{l}
F_{k} \\
F_{r}
\end{array}\right] \varepsilon_{t} e \\
\Psi:=F \rho^{*}
\end{gathered}
$$

onde $F$ é uma matriz não singular, $F_{k}$ é uma matriz de dimensão $k \times n$ e $F_{r}$ é uma matriz de dimensão $r \times n$.

Note que temos estabelecido que o componente estacionário em (6.2), $\Phi(L)\left(v_{t}+\Psi D_{t}\right)$, é função, por um lado, dos choques que ocorrem no componente não estacionário $\tau_{t}$ ou seja $\varphi_{t} e$, por outro lado, de outros choques independentes, representados por $\psi_{t}$.

Por outra parte, $v_{t}$ foi escrito como uma transformação linear $F$ de $\boldsymbol{\varepsilon}_{t}$, e $\Psi$ como uma transformação linear $F$ de $\rho^{*}$. Isto é consequência da própria derivação da representação de Tendências Comuns partindo da representação de Wold, como foi discutido no capítulo 4, seção 4.2 .

Defina :

$$
R(L):=C(L) F^{-1}
$$

Então, substituindo (6.4) e (6.5) em (6.1), obtemos :

$$
\begin{aligned}
\Delta \chi_{t} & =\delta+C(L)\left[\varepsilon_{t}+\rho^{*} D_{t}\right] \\
& =\delta+R(L)\left[v_{t}+\Psi D_{t}\right]
\end{aligned}
$$

Resumindo, em (6.6) temos os resíduos $v_{t}$, onde $\varphi_{t}$ denota a inovação $k$-dimensional das tendências comuns, $e$ o vetor r-dimensional $\psi_{t}$ pode ser considerado como o vetor das inovações transitórias.

O cálculo da função de resposta de impulsos se resume a calcular $R(L)$ e $\Psi$. Sendo que já possuimos $C(L)$ e $\rho^{*}$, como foi mostrado em capítulos anteriores, precisamos agora calcular a matriz F.

Já sabemos, por $(5.6)$ que $\mathrm{K} \varphi_{\mathrm{t}}=\mathrm{C}(1) \varepsilon_{\mathrm{t}}$, então : 


$$
\begin{aligned}
& \mathrm{K} \varphi_{\mathrm{t}}=\mathrm{C}(\mathrm{l}) \varepsilon_{\mathrm{t}} \\
& (\mathrm{K} \cdot \mathrm{K})^{-1} \mathrm{~K}^{\prime} \mathrm{K} \varphi_{\mathrm{t}}=\left(\mathrm{K}^{\prime} \mathrm{K}\right)^{-1} \mathrm{~K}^{\prime} \mathrm{C}(\mathrm{l}) \varepsilon_{\mathrm{t}} \\
& \varphi_{\mathrm{t}}=\left(\mathrm{K}^{\prime} \mathrm{K}\right)^{-1} \mathrm{~K}^{\prime} \mathrm{C}(\mathrm{l}) \varepsilon_{\mathrm{t}} \quad \therefore \quad \mathrm{F}_{\mathrm{K}}=\left(\mathrm{K}^{\prime} \mathrm{K}\right)^{-1} \mathrm{~K}^{\prime} \mathrm{C}(\mathrm{l})
\end{aligned}
$$

Para o cálculo de $F_{r}$, devemos impor uma segunda restrição adicional : a independência das inovações permanentes $e$ transitórias. Então, levando em conta (6.7) :

$$
\mathrm{E}\left[\varphi_{\mathrm{t}} \psi_{\mathrm{t}}^{\prime}\right]=\mathrm{E}\left[\left(\mathrm{K}^{\prime} \mathrm{K}\right)^{-1} \mathrm{~K}^{\prime} \mathrm{C}(\mathrm{l}) \varepsilon_{\mathrm{E}} \varepsilon_{\mathrm{t}}^{\prime} \mathrm{F}_{\mathrm{r}}^{\prime}\right]=\left(\mathrm{K}^{\prime} \mathrm{K}\right)^{-1} \mathrm{~K}^{\prime} \mathrm{C}(\mathrm{l}) \Sigma \mathrm{F}_{\mathrm{r}}^{\prime}=0
$$

Por outro lado, como demostrado por Engle e Granger (1987), C(1) $\gamma=0$. Então :

$$
F_{r}=Q^{-1} \gamma^{\prime} \Sigma^{-1}
$$

poderá satisfazer (6.8). A matriz $Q$, de dimensão $r \times r$ pode ser escolhida de modo qua a matriz de covariâncias das innovações transitórias $E\left[\psi_{t} \psi^{\prime}{ }_{t}\right]$ seja diagonal, ou identidade. Neste último caso, teremos $E\left(v_{t} v_{t}{ }_{t}\right)=I_{n}$.

\subsection{Exemplo}

Continuando com o exemplo desenvolvido nos capítulos anteriores, partindo da representação de Wold :

$$
\left[\begin{array}{l}
\Delta \chi_{1 t} \\
\Delta \chi_{2 t} \\
\Delta \chi_{3 t}
\end{array}\right]=\left[\begin{array}{l}
\delta_{1} \\
\delta_{2} \\
\delta_{3}
\end{array}\right]+C(L)\left\{\left[\begin{array}{l}
\varepsilon_{1 t} \\
\varepsilon_{2 t} \\
\varepsilon_{3 t}
\end{array}\right]+\left[\begin{array}{c}
\rho_{1}^{*} \\
\rho_{2}^{*} \\
\rho_{3}^{*}
\end{array}\right] D_{t}\right\}
$$

usando $(6.4),(6.5)$ e $(6.6)$ podemos escrever :

$$
\left[\begin{array}{l}
\Delta \chi_{1 \mathrm{t}} \\
\Delta \chi_{2 \mathrm{t}} \\
\Delta \chi_{3 \mathrm{t}}
\end{array}\right]=\left[\begin{array}{l}
\delta_{1} \\
\delta_{2} \\
\delta_{3}
\end{array}\right]+\mathrm{R}\left(\mathrm{L}_{\mathrm{L}}\right)\left\{\left[\begin{array}{l}
v_{1 \mathrm{t}} \\
v_{2 \mathrm{t}} \\
v_{3 \mathrm{t}}
\end{array}\right]+\left[\begin{array}{l}
\Psi_{1} \\
\Psi_{2} \\
\Psi_{3}
\end{array}\right] \mathrm{D}_{\mathrm{t}}\right\}
$$


onde:

$\mathrm{R}(\mathrm{L})=\mathrm{C}(\mathrm{L}) \mathrm{F}^{-1}, \quad\left[\begin{array}{l}v_{1 \mathrm{t}} \\ v_{2 \mathrm{t}} \\ v_{3 \mathrm{t}}\end{array}\right]=\mathrm{F}\left[\begin{array}{l}\varepsilon_{1 \mathrm{t}} \\ \varepsilon_{2 \mathrm{t}} \\ \varepsilon_{3 \mathrm{t}}\end{array}\right],\left[\begin{array}{l}\Psi_{1} \\ \Psi_{2} \\ \Psi_{3}\end{array}\right]=\mathrm{F}\left[\begin{array}{l}\rho_{1}^{*} \\ \rho_{2}^{*} \\ \rho_{3}^{*}\end{array}\right]$ e $\mathrm{F}=\left[\begin{array}{lll}f_{11} & f_{12} & f_{13} \\ f_{21} & f_{22} & f_{23} \\ f_{31} & f_{32} & f_{33}\end{array}\right]$

Usando (6.4), podemos escrever :

$$
\left[\begin{array}{l}
v_{1 \mathrm{t}} \\
v_{2 \mathrm{t}} \\
v_{3 \mathrm{t}}
\end{array}\right]=\left[\begin{array}{l}
\varphi_{1 \mathrm{t}} \\
\varphi_{2 \mathrm{t}} \\
\psi_{1 \mathrm{t}}
\end{array}\right]=\mathrm{F}\left[\begin{array}{l}
\varepsilon_{1 \mathrm{t}} \\
\varepsilon_{2 \mathrm{t}} \\
\varepsilon_{3 \mathrm{t}}
\end{array}\right]=\left[\begin{array}{lll}
\mathrm{f}_{11} & \mathrm{f}_{12} & \mathrm{f}_{13} \\
f_{21} & f_{22} & f_{23} \\
\hdashline f_{31} & f_{32} & f_{33}
\end{array}\right]\left[\begin{array}{l}
\varepsilon_{1 \mathrm{t}} \\
\varepsilon_{2 \mathrm{t}} \\
\varepsilon_{3 \mathrm{t}}
\end{array}\right]
$$

onde definimos :

$$
F_{k}=:\left[\begin{array}{lll}
f_{11} & f_{12} & f_{13} \\
f_{21} & f_{22} & f_{23}
\end{array}\right] \text { e } \quad F_{r}=:\left[\begin{array}{lll}
f_{31} & f_{32} & f_{33}
\end{array}\right]
$$

Usando (6.7), e as definições em (5.24), estimamos $F_{k}$ :

$$
F_{k}=\left[\begin{array}{lll}
f_{11} & f_{12} & f_{13} \\
f_{21} & f_{22} & f_{23}
\end{array}\right]=\left(K^{\prime} K\right)^{-1} K^{\prime} C(l)=\left[\begin{array}{lll}
k_{11} & k_{12} & k_{13} \\
k_{21} & k_{22} & k_{23}
\end{array}\right]\left[\begin{array}{lll}
c_{11} & c_{12} & c_{13} \\
c_{21} & c_{22} & c_{23} \\
c_{31} & c_{32} & c_{33}
\end{array}\right]
$$

Para estimar $F_{r}$, escreva :

$$
F_{r}=\left[\begin{array}{lll}
f_{31} & f_{32} & f_{33}
\end{array}\right]=Q^{-1} \gamma \Sigma^{-1}=\left[q_{1}\right]\left[\begin{array}{lll}
\gamma_{11} & \gamma_{21} & \gamma_{31}
\end{array}\right]\left[\begin{array}{lll}
\sigma_{11} & \sigma_{12} & \sigma_{13} \\
\sigma_{21} & \sigma_{22} & \sigma_{23} \\
\sigma_{31} & \sigma_{32} & \sigma_{33}
\end{array}\right]^{-1}
$$

Neste caso, $q_{1}$ pode assumir qualquer valor, ou um valor que assegure que a matriz de covariância $E\left(v_{t} v_{t}^{\prime}\right)$ seja $I_{n}$. 


\section{Capítulo 7 \\ Uma Aplicação da Metodologia a Séries Macroeconômicas da França e dos EUA}

Neste capítulo proporcionamos um exemplo de aplicação das técnicas descritas nos capitulos anteriores para um vetor compreendendo as seguintes variáveis : preços e taxas de juros na França e nos EUA, e a taxa de câmbio FFr/US\$.

Na seção 7.1 falaremos brevemente das teorias econômicas que servirão de base para o modelo, em particular da teoria da Paridade de Poder de Compra e da Paridade das Taxas de Juros não Coberta.

Na seção 7.2, após uma análise preliminar dos dados, abordaremos a estimação de um modelo VAR(2), com as séries índice de preços por atacado e taxas de juros de curto prazo na França e nos EUA e tipo de câmbio FFr/US\$. As eventuais relações de co-integração das variáveis existentes serão analisadas e estimadas. A partir das características do espaço de co-integração hipotetizadas, serão calculadas as representações de MCE, VAR restrito, Wold e de Tendências Comuns. Finalmente, a função de respostas de impulsos no contexto do modelo de Tendências Comuns é analisada.

\subsection{Uma descrição das Teorias da Paridade do Poder de Compra e da Paridade de Taxas de Juros não Coberta}

O estudo das relações entre preços, taxas de juros e tipo de câmbio tem sido abordado na literatura econômica por vários modelos.

Um dos enfoques tradicionais tem sido a "teoria da paridade de poder de compra"(PPC), que basicamente relaciona as variações percentuais da taxa de câmbio da moeda do país A pela moeda do país $B$ ao diferencial de inflação do país A com o país B:

$\inf f_{A}-i n f_{B}=\Delta T C$

onde :

$\inf _{\mathrm{A}}$ : taxa de inflação no país A 
inf $_{B}$ : taxa de inflação no país $B$

$\Delta \mathrm{TC}$ : variação percentual da taxa de câmbio da moeda do país A pela moeda do país B

Deste modo, os termos de intercâmbio dos dois países mantém-se constantes. Muitos estudos tem tentado encontrar na teoria da PPC a explicação dos movimentos de curto prazo do tipo de câmbio, tendo funcionado de maneira satisfatória somente nos casos onde os diferenciais de inflação são realmente grandes, sendo que muitos outros fatores afetam muito mais rapidamente os tipos de câmbio no curto prazo. No longo prazo, no entanto, existem maiores evidências de que a PPC se verifica. Neste estudo testaremos se tal relação de longo prazo tem um papel significativo para a taxa de câmbio do franco francês com relação ao dólar norte-americano, assim como para as taxas de juros e preços na França e nos EUA.

Na relação taxa de câmbio com taxas de juros, será testada a teoria da "paridade da taxa de juros não coberta" (PJNC, Frankel, 1979), que assume que o diferencial da taxa de juros do país A com o país B é igual ao diferencial percentual entre a taxa de câmbio esperada e a taxa de câmbio observada da moeda do país A pela moeda do país B. Em outras palavras, um país onde as expectativas com relação à taxa de câmbio são de depreciação de sua moeda terá as taxas de juros acima do "normal", sendo que um país com expectativas de valorização de sua moeda, terá taxas de juros abaixo do "normal". Assumindo que a variação da taxa de câmbio está dada pela diferença percentual entre a taxa esperada é a observada, temos a seguinte relação:

$r_{\mathrm{A}}-r_{\mathrm{B}}=\Delta \mathrm{TC}$

onde :

$r_{\mathrm{A}}$ : taxa de juros do país A

$r_{B}$ : taxa de juros do país $B$

$\Delta T C$ : variação percentual da taxa de câmbio da moeda do país A pela moeda do país B

Novamente este último enfoque será testado em termos de relação de longo prazo para o desenvolvimento do modelo a ser estimado nas próximas seções deste capítulo. 
As relações $(7.1)$ e (7.2) sugerem, para um $\chi_{t} \sim \mathrm{CI}(1,1)$, a formulação de um modelo de Mecanismo de Correção de Erro do tipo :

\section{Preços :}

$$
\begin{aligned}
& \Delta \mathrm{p}_{\mathrm{At}}=\mathrm{f}_{\mathrm{pA}}\left(\Delta \chi_{\mathrm{t}}\right)+\gamma_{1}\left(\mathrm{mce}_{\mathrm{ppc}}\right)_{\mathrm{t}-1}+\gamma_{2}\left(\text { mce }_{\mathrm{pjnc}}\right)_{\mathrm{t}-1}+\varepsilon_{\mathrm{pAt}} \\
& \Delta \mathrm{p}_{\mathrm{Bt}}=\mathrm{f}_{\mathrm{pB}}\left(\Delta \chi_{\mathrm{t}}\right)+\gamma_{3}\left(\text { mce }_{\mathrm{ppc}}\right)_{\mathrm{t}-1}+\gamma_{4}\left(\text { mce }_{\mathrm{pjnc}}\right)_{\mathrm{t}-1}+\varepsilon_{\mathrm{pBt}}
\end{aligned}
$$

Taxas de câmbio:

$$
\Delta \mathrm{e}_{\mathrm{ABt}}=\mathrm{f}_{\mathrm{e}}\left(\Delta \chi_{t}\right)+\gamma_{5}\left(\mathrm{mce}_{\mathrm{ppc}}\right)_{\mathrm{t}-1}+\gamma_{6}\left(\mathrm{mce}_{\mathrm{pjnc}}\right)_{\mathrm{t}-1}+\varepsilon_{\mathrm{ABt}}
$$

Taxas de Juros :

$$
\begin{aligned}
& \Delta j_{A t}=f_{j A}\left(\Delta \chi_{t}\right)+\gamma_{7}\left(m_{p e} e_{p p c}\right)_{t-1}+\gamma_{8}\left(m_{c e} e_{p j n c}\right)_{t-1}+\varepsilon_{j A t} \\
& \Delta j_{B t}=f_{j B}\left(\Delta \chi_{t}\right)+\gamma_{9}\left(m_{c e} e_{p p c}\right)_{t-1}+\gamma_{10}\left(m_{c e} e_{p n c}\right)_{t-1}+\varepsilon_{j B t}
\end{aligned}
$$

onde $\chi_{t}=\left[\begin{array}{lllll}p_{A t} & P_{B t} & e_{A B t} & j_{A t} & j_{B t}\end{array}\right]^{\prime}$ é 0 vetor de variáveis endógenas nível de preços no país $A$ e $B$ em logaritmos, taxa de câmbio da moeda do país A pela moeda do país $B$, em logaritmos, e taxas de juros nos países $A$ e $B$. Os vetores $f_{i t}$ representam as relações de curto prazo, sendo que os coeficientes $\gamma_{i}$ representam os pesos das relações de longo prazo. As relações de longo prazo estão dadas por $\alpha_{i}^{\prime} \chi_{t}=$ mce $_{i}$, onde $\alpha_{i}^{\prime}$ para $i=1,2$, são os vetores de co-integração. Temos :

$$
\begin{aligned}
& \text { mce }_{\mathrm{ppc}}=\alpha_{1}{ }^{\prime} \chi_{\mathrm{t}}=\left(\mathrm{p}_{\mathrm{At}}-\mathrm{p}_{\mathrm{Bt}}-\mathrm{e}_{\mathrm{AB}}\right) \\
& \mathrm{mce}_{\mathrm{pjnc}}=\alpha_{2}^{\prime} \chi_{\mathrm{t}}=\left(\Delta \mathrm{e}_{\mathrm{ABt}}-\left(j_{\mathrm{At}}-j_{\mathrm{Bt}}\right)\right)
\end{aligned}
$$

Note que, na relação mce $_{\text {pjnc }}$ em (7.6), na solução de longo prazo, $\Delta \mathrm{e}_{\mathrm{ABt}}=0$. Assim, as relações de longo prazo relevantes nas equações do sistema são a PPC e o diferencial das taxas de juros. Isto significa que os vetores de co-integração estão no espaço gerado por uma matriz do tipo : 


$$
\alpha=\left[\begin{array}{ll}
\alpha_{1} & \alpha_{2}
\end{array}\right]=\left[\begin{array}{cc}
-1 & 0 \\
1 & 0 \\
1 & 0 \\
0 & -1 \\
0 & 1
\end{array}\right]
$$

\subsection{Resultados empíricos}

\subsubsection{Propriedades de Integração e Co-integração dos Dados}

Os dados ${ }^{1}$ (apresentados no apêndice E) são observações trimestrais, definindo as variáveis endógenas do modelo da seguinte forma :

ipafr = índice de preços por atacado na França, 1985=100, em logaritmos;

ipaus = índice de preços por atacado nos USA, 1985=100, em logaritmos;

ffus = taxa de câmbio FFr/US\$ (francos por dólar), média do mês, em logaritmos;

jurfr = logaritmo(1+taxa de juros a curto prazo na França/100), onde a taxa de juros a curto prazo é a cotada no Euromercado, para bônus do tesouro francês, prazo 3 meses, média do mês;

tbill = logaritmo(1+taxa de juros a curto prazo nos USA/100), onde a taxa de juros a curto prazo corresponde ao rendimento dos "t-bills', bônus do governo do Tesouro norteamericano, prazo 3 meses, média do mês.

Indagaremos o efeito da variável exógena preço do petróleo, definido da seguinte forma :

doilp $=1^{\text {a }}$ diferença do logaritmo do preço internacional do petróleo, média do mês.

O período de estudo é de 1970:01 até 1993:04.

No gráfico 1, são apresentados os dados correspondentes às variáveis endógenas, em níveis e em $1^{\text {as }}$ diferenças :

${ }^{1}$ Fonte : OCDE 


\section{Gráfico 7.1}
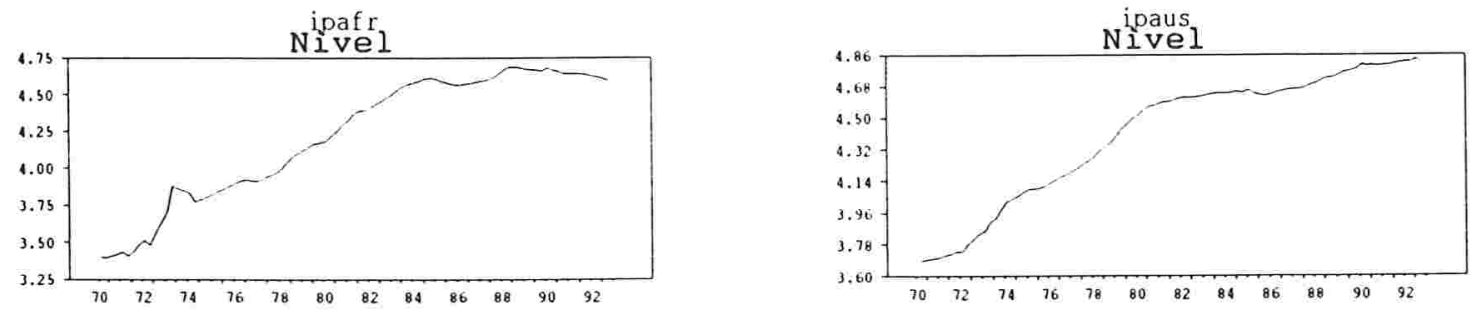

Diferencas

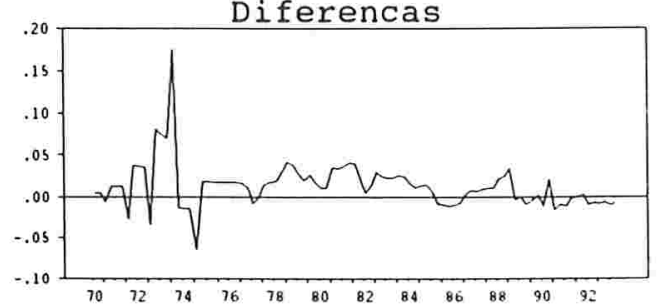

Diferencas
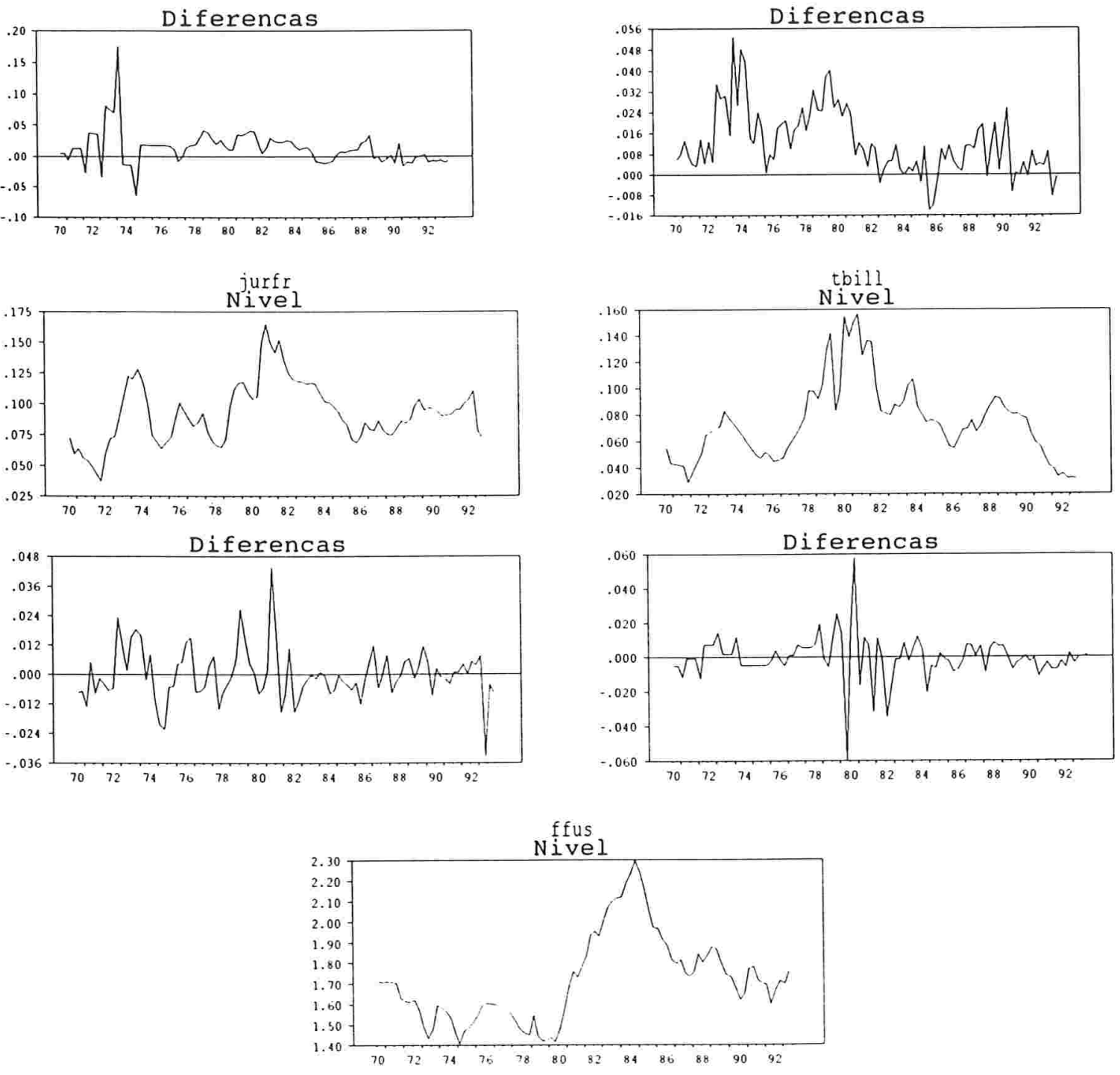

Diferencas

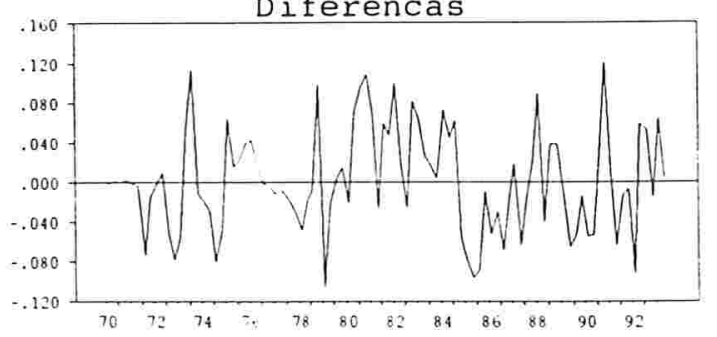


A análise univariada das séries mediante as funções de autocorrelação amostral sugerem que as séries são I(1), ou seja, sendo que as séries em níveis não são estacionárias, o que já vimos claramente no gráfico 1, as primeiras diferenças são estacionárias (ver anexo deste capítulo). Os testes de Dickey Fuller aplicados sobre as primeiras diferenças das séries rejeitam a hipótese da existência de raízes unitárias ao 0,05 nível de significância (valor crítico para regressão com constante, sem tendência: $-2,89$; valor crítico para regressão com constante e tendência: $-3,45)$, sugerindo que as $1^{\text {as }}$ diferenças são I $(0)$.

\section{Quadro 7.1}

Estatísticas de Dickey-Fuller para Testes de Raízes Unitárias nas $1^{a s}$
diferenças das variáveis do vetor $\chi_{t}$
$x_{t}$
$\tau_{1}$ (com constante) $\tau_{2}$ (com constante
e tendência)

No entanto, a análise gráfica sugere que estamos em um caso limite de estacionariedade. De fato, os gráficos sugerem não estacionariedade da variância, e não tanto da média (com a exceção tal vez de ipaus). Assim, diferenciar as séries uma segunda vez não seria um método adequado para remover a não estacionariedade. Foi seguido então o enfoque de Johansen \& Juselius (1992) em sua análise da taxa de câmbio da Libra esterlina com relação a uma cesta de moedas. As variáveis foram condicionadas às mudanças do preço de petróleo, que foram consideradas como a origem da não estacionariedade da variância das séries. Adicionalmente, foram introduzidas variáveis dummy para períodos de grande instabilidade. Este procedimento, ajudou também, como veremos, a induzir normalidade nos resíduos.

Por fim, no gráfico 7.2 são apresentadas as relações de co-integração hipotetizadas : 


\section{Gráfico 7.2}

1a Relação de Cl ffus-ip a fr tipaus

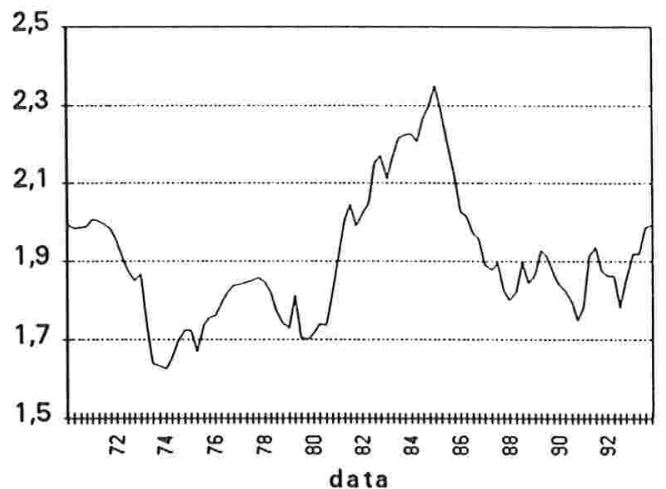

2a Relação de Cl jurf́r-tbill

Gráficamente, a $1^{a}$ relação (ffus-ipafr+ipaus) não apresenta uma tendência, mas ela fica longe da média no período 80-85, coincidindo com a era Volcker nos EUA, com aplicação de políticas monetárias fortemente restritivas naquele país, combinadas a um crescente déficit orçamentário, o que fez disparar o dólar. A $2^{a}$ relação (jurfr-tbill), apresenta uma ligeira tendência ascendente no final do período de estudo. No quadro 7.2 são apresentados os testes de Dickey Fuller para as relações de co-integração acima sugeridas. Agregamos igualmente os testes de raízes unitárias para outras relações possíveis, observadas nos mercados financeiros :

$3^{a}$ relação: ffus-tbill+jurfr, que representa em certo sentido a tese contrária à teoria de PJNC, afirmando que o dólar tende a subir quando o diferencial das taxas de juros nos USA com relação às taxas de juros na França aumenta, devido ao incremento da procura do dólar;

$4^{a}$ relação: ffus-tbill+ipaus+jurfr-ipafr, a mesma idéia que a apresentada na $3^{a}$ relação, mas em função das taxas de juros reais (taxas de juros nominais - inflação)

Nos testes de Dickey Fuller, a hipótese nula de presença de raiz unitária é rejeitada somente na $2^{a}$ relação (diferencial das taxas de juros), ao nível de significância de 0,05 (valor crítico para 100 observações, em uma regressão com constante, sem tendência : $-2,89$; na regressão com constante e tendência, a tendência não foi estatísticamente significativa). Nos outros casos, a hipótese nula de raízes unitárias não é rejeitada. No entanto, voltaremos a analizar mais tarde estas últimas relações, com os testes de Johansen 
\& Juselius. Em alguns casos, serão testadas hipóteses sobre a existência de outros tipos de combinações lineares estacionárias de jurfr, tbill e ffus.

o procedimento de Johansen, que estimará primeiro o espaço de co-integração de maneira irrestrita, permitirá realizar diferentes tipos de testes de restrições sobre os vetores de co-integração. Obteremos assim mais informação sobre os vetores geradores daquele espaço, e se estes são compatíveis com as teorias econômicas esboçadas acima.

\section{Quadro 7.2}

Estatísticas de Dickey-Fuller Aumentadas ( 5 lags) para Testes de Raízes Unitárias nas relações de co-integração

\begin{tabular}{lcc}
\multicolumn{1}{c}{$z_{t}$} & $\tau_{1}$ (com constante) & $\begin{array}{c}\tau_{2} \text { (com constante } \\
\text { e tendencia) }\end{array}$ \\
& & $-2,20$ \\
ffus-ipafr+ipaus & $-2,53$ & $-3,26$ \\
jurfr-tbill & $-3,13$ & $-2,17$ \\
ffus+jurfr-tbill & $-1,81$ & $-2,58$ \\
ffus-tbill+ipaus+jurfr-ipafr & $-2,32$ &
\end{tabular}

\subsubsection{Estimação da Representação MCE}

o vetor em estudo :

$$
\chi_{t}=\left[\begin{array}{c}
\text { ipafr }_{t} \\
\text { ipaus }_{t} \\
\text { ffus }_{t} \\
\text { jurfr }_{t} \\
\text { toill } \\
t
\end{array}\right]
$$

tem uma representação $\operatorname{VAR}(p)$ :

$$
A(L) \chi_{t}=\rho+\rho_{1}^{*} D_{1 t}+\rho_{2}^{*} D_{2 t}+\rho_{3}^{*} D_{3 t}+\sum_{j} \omega_{j} Z_{j}+\varepsilon_{t}
$$


onde $\rho$ é um vetor de constantes, os $\rho_{i}{ }^{*}$ são vetores de parâmetros correspondentes às dummies sazonais $D_{i t}$ os $\omega_{j}$ são vetores de parâmetros correspondentes às variáveis exógenas do modelo, e $\varepsilon_{\mathrm{t}}$ é o vetor dos erros. As variáveis exógenas do modelo são o preço de petróleo (1 ${ }^{\text {a }}$ diferença do logaritmo, doilp), e variáveis dummy que tomam conta de períodos de grande instabilidade, em particular com relação às taxas de juros, para determinados trimestres em 74, 80, 81 (correspondentes aos choque dos preços do petróleo, e a aplicação de políticas monetárias altamente restritivas em USA) e 93 (correspondente à crise no Sistema Monetário Europeu).

Uma vez identificado o espaço de co-integração, será obtida a representação do Mecanismo de Correção de Erro para o processo estocástico $\left\{\chi_{t}\right\}$ :

$$
A^{*}(L) \Delta \chi_{t}=\rho+\rho_{1}^{*} D_{1 t}+\rho_{2}^{*} D_{2 t}+\rho_{3}^{*} D_{3 t}-\gamma \alpha^{\prime} \chi_{t-1}+\sum_{j} \omega_{j} Z_{j t}+\varepsilon_{t}
$$

onde $\alpha$ é o vetor de co-integração, $\gamma$ é o vetor dos pesos, $A^{*}(L)$ é um polinômio matricial tal que $A(L)=A^{*}(L)(1-L)+A(l) L$ com a especificação já descrita no capítulo 2.

Antes de obter as estimativas finais de (7.9) e (7.10), é preciso decidir a ordem do $\operatorname{VAR}(p)$. O quadro 7.3. apresenta os critérios de informação FPE (Final prediction Error), AIC (Akaike's Information Criterion), HQ (Hannan-Quinn Criterion) e SC (Shwarz Criterion) para VAR estimados da ordem 1 até 4. Os critérios FPE e AIC escolhem $p=4$, enquanto que o critérios HQ escolhe $\mathrm{p}=2$ e $\mathrm{SC}$ escolhem $\mathrm{p}=1$.

\section{Quadro 7.3}

Seleção da Ordem do modelo $\operatorname{VAR}(p)$ irrestrito Critérios de Informação

\begin{tabular}{|c|c|c|c|c|}
\hline LAGS & 1 & 2 & 3 & 4 \\
\hline FPE & $0,143-\mathrm{E} 18$ & $0,105 \mathrm{E}-18$ & $0,986 \mathrm{E}-19$ & $0,913 E-19$ \\
\hline AIC & $-43,388$ & $-43,705$ & $-43,780$ & $-43,880$ \\
\hline $\mathrm{HQ}$ & $-43,056$ & $-43,097$ & $-42,895$ & $-42,719$ \\
\hline $\mathrm{SC}$ & $-42,566$ & $-42,198$ & $-41,857$ & $-41,002$ \\
\hline
\end{tabular}

Os critérios FPE e AIC são práticamente equivalentes para pequenas e moderadas amostras. Por outro lado, estes dois critérios não são estimadores consistentes da verdadeira ordem do processo, e tendem a sobre-estimar o valor de p. o crítério HQ é consistente e SC é super-consistente. Como o 
tamanho da amostra é relativamente grande, escolhemos os critérios HQ e SC (ver Lutkepohl, págs. 130-131). Para escolher entre os dois lags 2 e 1 , foi calculado o teste de verossimilhança para $H_{\circ}: p=1$ vs $H_{a} p=2$.

O teste $\lambda_{\text {MV }}$, que atinge um valor de 59,248, e que tem uma distribuição assintótica $\chi^{2}(25)$ sob a $H_{0}$, rejeita a hipótese nula ao nível de significância de 0,05. Assim, foi escolhida a estimação de um VAR de ordem 2.

A equação (7.10) foi estimada diretamente usando o procedimento de Johansen, (descrito no apêndice D) ${ }^{2}$, que é um estimador de máxima verossimilhança, baseado na hipótese de que $\left\{\boldsymbol{\varepsilon}_{\mathrm{t}}\right\}$ é um processo Gaussiano. O quadro 7.4 apresenta alguns resultados e testes com relação aos resíduos.

Os testes Q de Box \& Pierce, para 21 lags, não rejeitam a hipótese nula de ausência de autocorrelação dos resíduos, ao $90 \%$ de significância, sendo que o valor dos testes são inferiores ao valor crítico $\chi_{0,90}(19)=27,2$, em todos os casos. Com relação à presença de heterocedasticidade, a $H_{0}$ de ausência de um processo $\mathrm{ARCH}$ de $2^{\mathrm{a}}$ ordem não é rejeitada ao nível de significância de 0,10 para ipaus, ffus, jurfr e tbill, sendo que os testes são inferiores ao valor crítico

$\chi_{0,90}(2)=4,61$. Com relação a ipafr, a hipótese nula de homocedasticidade também não é rejeitada, embora a um nível de significância menor $(\alpha=0,001)$. No teste de normalidade de Jarque-Bera, a $H_{\circ}$ de que os resíduos seguem um processo gaussiano não é rejeitada para todas as equações, ao nível de significância de 0,10 para ipaus, ffus e jurfr, e de 0,01 para ipafr e tbill.

\section{Quadro 7.4}

Análise Residual Univariada

\begin{tabular}{|c|c|c|c|c|c|}
\hline Equ & Média & $V A R$. & MAX. & MIN. & $S Q R$ \\
\hline a & 00000 & 0,00025384 & 0,04730328 & $-0,05417043$ & 0,6 \\
\hline ip & 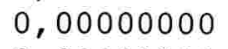 & & 319 & 2167015 & 0 , \\
\hline ffus & 0,00000000 & 0,00178902 & 0, & -0 & 0 , \\
\hline jurfr & 0,0000000 & 0,00005369 & 0,02100005 & $-0,1542256$ & 0,52074102 \\
\hline tbill & 0,00000000 & 0,00005944 & 0,01893760 & $-0,02760327$ & 0,62446135 \\
\hline
\end{tabular}

${ }^{2} \mathrm{O}$ programa utilizado foi CATS (Cointegration Analysis for Time Series), elaborado por Johansen \& Juselius, e funciona como um procedimento do programa "RATS". 
Quadro 7.4 (continuação)

Análise Residual Univariada

\begin{tabular}{lcrrrr} 
Equação & $B-P . Q(21)$ & ARCH(2) & asimetria & ex.curt. & J-B.Norm. \\
\cline { 1 - 3 } & 24,388 & 10,727 & $-0,093$ & 1,840 & 13,394 \\
ipaus & 23,901 & 0,284 & 0,049 & 0,982 & 3,816 \\
ffus & 16,381 & 3,575 & 0,094 & $-0,024$ & 0,140 \\
jurfr & 23,243 & 1,730 & 0,361 & 0,670 & 3,805 \\
tbill & $14 ., 75$ & 4,587 & $-0,324$ & 1,509 & 10,562
\end{tabular}

A normalidade e ausência de autocorrelação dos resíduos são hipóteses fundamentais no que tange aos testes que serão apresentados a seguir, todos eles baseados na verossimilhança da amostra, partindo da hipótese de que os erros possuem uma distribuição normal, independente e homocedástica.

A introdução das dummies que "tomam conta" dos períodos de instabilidade das variáveis era essencial para induzir normalidade nos resíduos. Com efeito, nas estimações preliminares do modelo, que não consideravam as variáveis dummies mencionadas, os testes rejeitaram normalidade dos resíduos (por excesso de curtose) e homocedasticidade, para ipafr, jurfr e tbill. Vendo os resultados do quadro 7.4, vemos que a performance dos testes para normalidade não é ainda muito satisfatória para ipafr e tbill.

Segundo Johansen (1992), a sensibilidade da análise à escolha da ordem do lag, e à distribuição dos resíduos podem ser investigados por experimentos Monte Carlo. De acordo com a análise assintótica (Johansen, 1988, 1991), os resultados limites valem se os resíduos admitem o Teorema Central do Limite, no sentido de que as somas acumuladas convergem à movimentos Brownianos.

\subsubsection{Análise do Espaço de Co-integração}

o quadro 7.5 apresenta a) as estimativas para os autovalores $\lambda_{1}>\lambda_{2} \ldots \lambda_{5}$ da expressão $\mathrm{S}_{10} \mathrm{~S}_{00}^{-1} \mathrm{~S}_{01}$ com relação a $\mathrm{S}_{11}$, conceito discutido no Apêndice D (varíáveis canônicas) , b) a estatística Lambdamax e c) a estatística do Traço.

Quadro 7.5

AUTOVALORES, LAMBDAMAX E ESTATITICA DO TRAÇO

$\begin{array}{lrrcrr}\text { Autovalores } & 0,418 & 0,365 & 0.210 & 0.108 & 0.016 \\ \text { Lambdamax } & 50,826 & 42,674 & 22.100^{*} & 10.692 & 1.482 \\ \text { Est. do Traço } & 127,773 & 76,947 & 34.273^{*} & 12.173 & 1.482\end{array}$


Ao nível de significância de 0,05, ambos os testes (usando a distribuições calculadas por Johansen \& Juselius, 1990) sugerem que os 3 primeiros autovalores são significativamente diferentes de 0 , ou seja que o espaço de co-integração é de dimensão 3 ( $r=3)$. Assim, $\alpha$ e $\gamma$ em (7.10) podem ser consideradas matrizes de dimensão $5 \times 3$. As estimativas das matrizes $\alpha$ normalizada e $\gamma$, uma vez assumido que o espaço de co-integração é de dimensão 3 , são apresentadas a seguir:

\section{Quadro 7.6}

\begin{tabular}{rrrrrr}
\multicolumn{4}{c}{$\alpha$} & \multicolumn{2}{c}{$\gamma$} \\
$\alpha_{1}$ & $\alpha_{2}$ & $\alpha_{3}$ & $\gamma_{1}$ & $\gamma_{2}$ & $\gamma_{3}$ \\
& & & & & \\
1,000 & 1,088 & $-0,034$ & 0,039 & 0,122 & 0,117 \\
$-1,228$ & $-1,109$ & 0,019 & $-0,038$ & 0,006 & 0,066 \\
$-0,601$ & $-0,250$ & 0,089 & $-0,134$ & 0,087 & 0,177 \\
3,114 & 1,000 & 1,000 & 0,022 & $-0,011$ & 0,140 \\
1,529 & $-2,537$ & $-0,563$ & 0,035 & $-0,002$ & 0,078
\end{tabular}

o quadro 7.7 exibe a matriz $\gamma \alpha^{\prime}$, e a matriz dos desvios padrão para $\gamma \alpha^{\prime}$ :

\section{Quadro 7.7 .}

$\gamma \alpha^{\prime}$

Desvios padrão para $\boldsymbol{\alpha}^{\prime}$

\begin{tabular}{|c|c|c|c|c|c|c|c|c|c|}
\hline 167 & $-0,180$ & $-0,043$ & 0,359 & $-0,316$ & 0,028 & 0,031 & 0,014 & 0,099 & 0,070 \\
\hline 03 & 0,041 & 0,02 & $-0,046$ & -0 & 0,011 & 0,013 & 0 , & 0,040 & \\
\hline 04 & 0,071 & 0,0 & -0 & & & & & 65 & \\
\hline 0,00 & $-0,011$ & 0,002 & 0,196 & . & 2 & 0 , & & & 0,030 \\
\hline 0,030 & $-0,039$ & $-0,013$ & 0,184 & 0,014 & 0,013 & 0,015 & 0,007 & 0,047 & 0,0 \\
\hline
\end{tabular}

A análise a seguir será então baseada na hipótese de que a dimensão do espaço de co-integração é 3 .

A interpretação do espaço de co-integração à luz das teorias discutidas na seção 7.1 é complexo. Podem ser feitos os seguintes comentários :

a) A primeira vista, a relação 1, a PPC, parece estar contida em $\alpha_{1}$ sendo que a relaçõo 2 de diferencial de taxas de juros esperada, implicada pela PJNC, estaria entrando em $\alpha_{2}$ e $\alpha_{3}$.

b) As relações 3 (ffus-tbill+jurfr) e 4 (ffustbill+jurfrtipaus-ipafr) podem estar entrando em $\alpha_{3}$. 
c) A relação de PPC pode também estar entrando em $\alpha_{3}$ Nesse caso $\alpha_{3}$ poderia estar contendo a relação 1 (PPC) e a relação 2 (diferencial das taxas de juros).

d) Os dois últimos valores do vetor $\alpha_{1}(3,114$ e 1,529) que representam uma média ponderada das taxas de juros, em vez de um diferencial, são de difícil interpretação econômica, resultado semelhante ao obtido por Johansen \& Juselius em seu estudo sobre a taxa de câmbio Libra/US\$ (1992).

Com relação a $\gamma$, que representa os pesos das relações de co-integração em cada uma das equações, e considerando o cálculo de $\gamma \alpha^{\prime}$ e os desvios padrões dessa matriz ${ }^{3}$ (com as estatísticas $t$ implícitas), apresentados no quadro 7.7, são feitos os seguintes comentários :

e) Considerando as matrizes $\gamma$ e $\gamma \alpha^{\prime}$, as relações de cointegração possuem um peso maior para as variáveis ffus e ipafr, seguidas por jurfr, tbill e ipaus, nessa ordem, aproximadamente.

f) Considerando a matriz dos desvios padrão, é interessante observar que no que tange à taxa de câmbio, a relação de longo prazo de peso estatísticamente significativa é uma combinação linear entre a taxa de câmbio e a taxa de juros nos EUA.

g) $\mathrm{Na}$ equação da taxa de juros da França, somente o peso de $\gamma_{3}$ parece significativamente diferente de 0 , e considerando a matriz $\gamma \alpha^{\prime}$ e seus desvios padrão, só as taxas de juros parecem estar entrando na relação de longo prazo nas taxas de juros na França.

h) Para as taxas de juros nos Estados Unidos, os pesos de $\gamma$ são baixos, embora estatísticamente significativos quando a matriz $\gamma \alpha^{\prime}$ e seus desvios padrão são considerados. Não tem explicação econômica o fato de que, para a equação da taxa de juros nos EUA, a taxa de juros da França seja estatísticamente significativa, sendo que a dos EUA não é.

i) De modo geral, considerando $\gamma \alpha^{\prime}$, a relação PPC parece estar presente em todas as equações, com exceção de jurfr. Em contraste, as taxas de juros não estão entrando nas equações do modo esperado. Na $2^{a}, 3^{a}$ e $5^{a}$ equações a média ponderada das taxas de juros, em vez dos diferenciais, parecem ser

3 Calculada pelo método Delta. 
relevantes. Em particular o diferencial não entra na $3^{a}$ equação, que é a da taxa de câmbio.

Com relação as estimativas de curto prazo da equação (7.10), estas são apresentadas a seguir :

\section{Quadro 7.8}

ESTIMATIVAS DOS COEFICIENTES DE CURTO PRAZO DA EQUAÇÃO (7.10)

\begin{tabular}{|c|c|c|c|c|}
\hline \multicolumn{5}{|c|}{ Matriz $\mathrm{A}_{1}{ }^{*}$} \\
\hline Ipa & ipaus & ffus & jurfr & (bill) \\
\hline 0,2 & 0,289 & $-0,095$ & 0,152 & $-0,073$ \\
\hline$-0,02$ & 0,182 & $-0,011$ & 0,117 & $-0,043$ \\
\hline 0,262 & $-1,559$ & 0,243 & $-0,709$ & 0,331 \\
\hline 0,07 & 0,023 & 0,017 & 0,319 & $-0,024$ \\
\hline & 0,279 & & & \\
\hline
\end{tabular}

Vetores $\omega$

\begin{tabular}{rrrrrr}
\multicolumn{7}{c}{ Vetores $\omega$} \\
(doilp & d74q 1 & d80q2 & d80 84 & d8 1q2 & d93q2 ) \\
0,028 & 0,168 & $-0,030$ & $-0,020$ & $-0,021$ & $-0,003$ \\
0,041 & $-0,004$ & $-0,005$ & $-0,001$ & 0,002 & 0,005 \\
$-0,075$ & 0,130 & $-0,009$ & 0,079 & 0,068 & $-0,009$ \\
0,009 & 0,004 & 0,003 & $-0,003$ & 0,041 & $-0,031$ \\
0,010 & 0,013 & $-0,062$ & 0,059 & 0,008 & 0,003
\end{tabular}

$\begin{array}{rrrc}\rho_{1}{ }^{*} & \rho_{2}{ }^{*} & \rho_{3}{ }^{*} & \rho \\ (\mathrm{D} 1 & \mathrm{D} 2 & \mathrm{D} 3 & \text { constante) } \\ -0,010 & 0,004 & -0,002 & -0,149 \\ -0,001 & -0,001 & -0,002 & 0,083 \\ 0,011 & 0,020 & 0,008 & 0,213 \\ -0,001 & -0,004 & -0,000 & -0,012 \\ -0,001 & 0,002 & 0,002 & -0,055\end{array}$

T-estatisticas

$\begin{array}{ccccc}2,6 & 1,3 & 2,3 & 0,8 & 0,4 \\ 0,8 & 2,0 & 0,6 & 1,4 & 0,6 \\ 1,2 & 2,6 & 2,2 & 1,3 & 0,7 \\ 2,1 & 0,2 & 1,0 & 3,7 & 0,3 \\ 1,4 & 2,6 & 2,5 & 2,1 & 1,4\end{array}$

T-estatísticas

$\begin{array}{llllll}1,7 & 7,0 & 1,6 & 1,1 & 1,1 & 0,2\end{array}$

$\begin{array}{llllll}6,0 & 0,4 & 0,6 & 0,1 & 0,3 & 0,7\end{array}$

$\begin{array}{llllll}1,7 & 2,0 & 0,2 & 1,6 & 1,3 & 0,2\end{array}$

$\begin{array}{llllll}1,3 & 0,4 & 0,3 & 0,4 & 4,8 & 3,9\end{array}$

$\begin{array}{llllll}1,3 & 1,1 & 6,8 & 6,6 & 0,8 & 0,3\end{array}$

T-estatisticas

$\begin{array}{llll}1,8 & 0,8 & 0,4 & 3,2 \\ 0,6 & 0,4 & 0,9 & 4,4 \\ 0,8 & 1,4 & 0,6 & 1,7 \\ 0,5 & 1,5 & 0,1 & 0,6 \\ 0,3 & 0,6 & 0,8 & 2,5\end{array}$

A matriz $\sum$ estimada onde $\sum=\mathrm{E}\left(\varepsilon_{\mathrm{t}} \varepsilon_{\mathrm{t}}{ }^{\prime}\right)$ é apresentada no quadro $7.9:$

Quadro 7.9

Matriz $\Sigma$

$0,3293663 \mathrm{E}-03$

$0,1739513 \mathrm{E}-06 \quad 0,4259602 \mathrm{E}-04$

$0,1789788 \mathrm{E}-03 \quad-0,3831643 \mathrm{E}-(0) \quad 0,1859411 \mathrm{E}-02$

$0,1271159 \mathrm{E}-04 \quad-0,2928637 \mathrm{E}-05 \quad-0,4366987 \mathrm{E}-05 \quad 0,5081584 \mathrm{E}-04$

0,1922124E-04 0,9713201E-05 0,1887375E-04 0,1232639E-04 0,6009888E-04

O gráfico 7.3 apresenta as relações de co-integração estimadas. As relações de co-integração são apresentadas na 
ordem dos autovalores do maior para o menor. Quanto maior o autovalor maior a correlação da relação de co-integração hipotetizada com as $1^{\text {as }}$ diferenças das variáveis do sistema. Se espera que as relações tenham aspecto de séries estacionárias, embora não sejam ruídos brancos. Os gráficos que apresentam $\alpha^{\prime} R_{1 t}\left(R_{1 t}\right.$ representa o resíduo da regressão de $\chi_{t}$ sobre $\Delta \chi_{t-1}, \ldots . \Delta \chi_{t-k+1}$, ver apêndice sobre método de Johansen) devem ser mais satisfatórios neste sentido, uma vez que estes representam as relações de co-integração corrigidas dos efeitos de curto prazo.

\section{Gráfico 7.3}
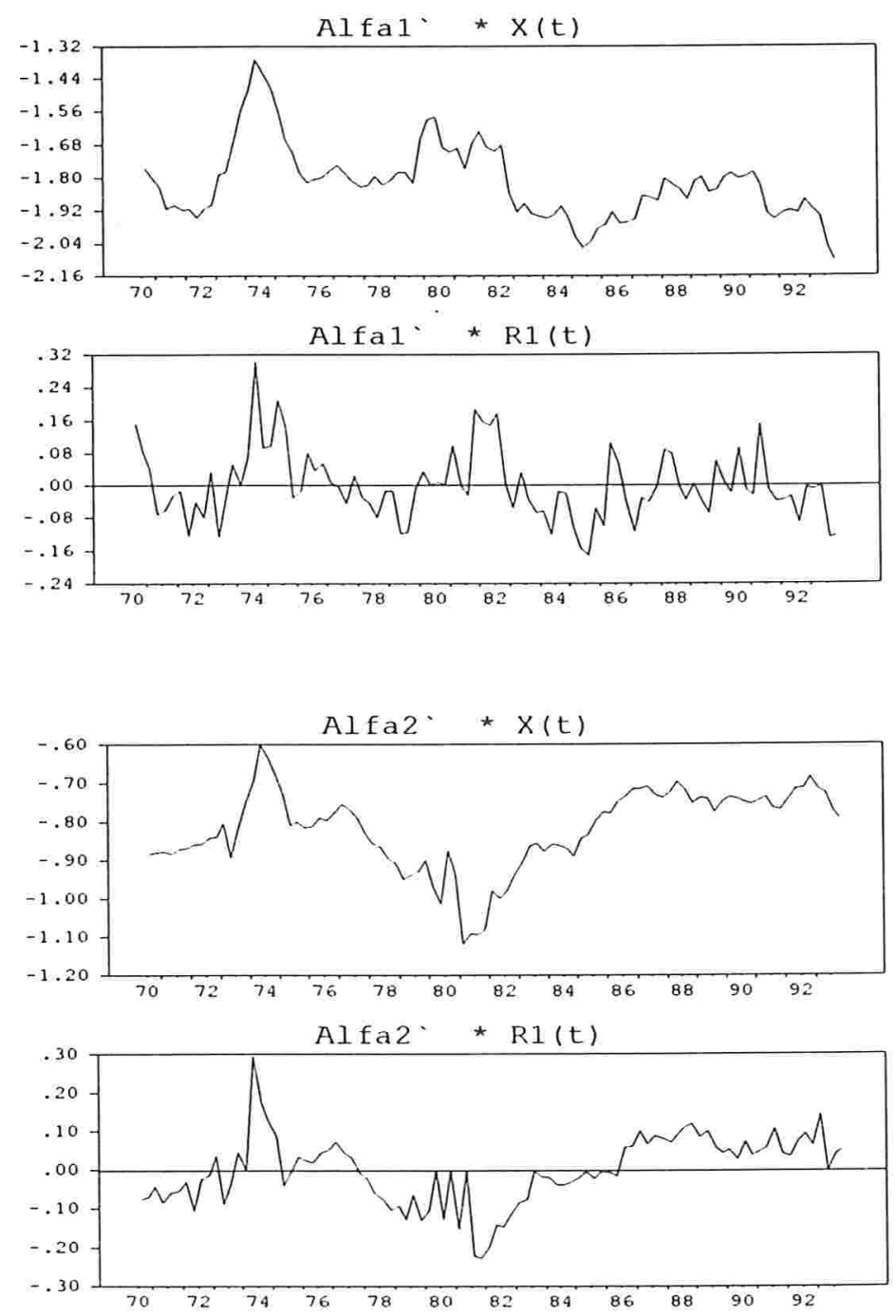


\section{Gráfico 7.3 (continuação)}
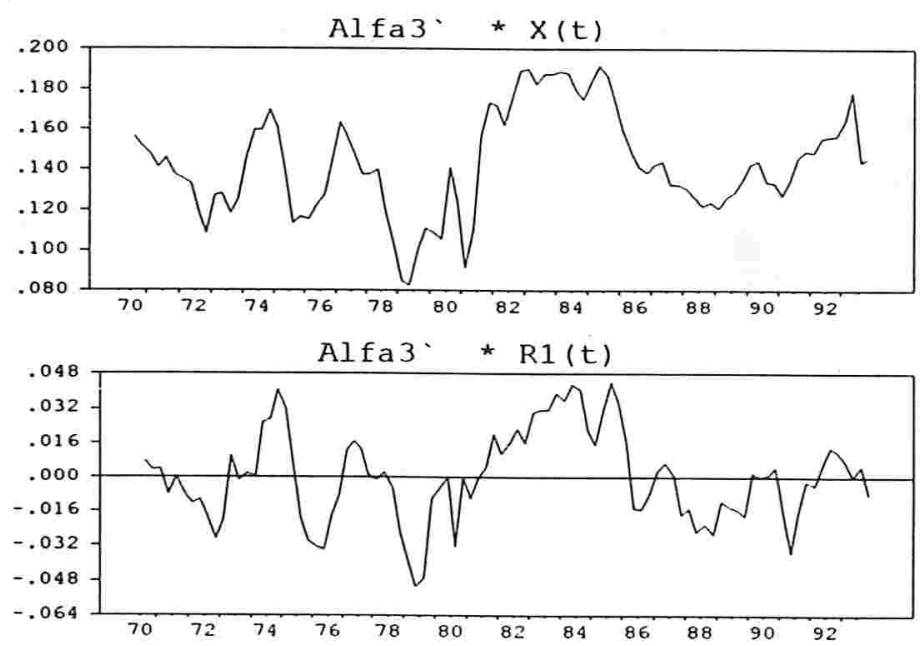

\subsubsection{Alguns Testes sobre Restrições do Espaço de Co- integração}

Os comentários feitos na seção anterior sugerem a aplicação de uma série de testes, usando a metodologia de Johansen, que compara modelos restritos com modelos irrestritos atravês da estatística da razão de verossimilhança (ver apêndice D). Em geral o teste da razão de verossimilhança é da forma :

$$
-2 \ln Q\left(\mathrm{H}_{0} \mid \mathrm{H}_{1}\right)=\mathrm{T} \ln \left\{\frac{\prod_{i=1}^{r}\left(1-\lambda_{\mathrm{i}}^{\mathrm{R}}\right)}{\prod_{\mathrm{i}=1}^{r}\left(1-\lambda_{\mathrm{i}}^{\mathbb{R}}\right)}\right\}=\mathrm{T}\left(\sum_{i=1}^{\mathrm{r}} \ln \left(1-\lambda_{\mathrm{i}}^{\mathrm{R}}\right)-\sum_{i=1}^{r} \ln \left(1-\lambda_{i}^{\mathbb{I R}}\right)\right)
$$

onde $T$ é o número de observações e os $\lambda_{i}{ }^{R R}$ e $\lambda_{i}{ }^{\text {IRR }}$ correspondem aos autovalores dos modelos restrito e irrestrito, respectivamente. A estatística (7.11) é distribuída assintóticamente de modo aproximado como uma $\chi^{2}(q)$, onde $q$ é o número de graus de liberdade que dependerá do número de restrições. Valores grandes da estatística rejeitam a hipótese nula de que o modelo restrito é o verdadeiro.

Com relação ao espaço de co-integração, foram realizados os seguintes testes:

a) $H_{0}$ : O vetor $\left[a_{i}-a_{i}-a_{i} * *\right]$, pertence ao espaço de cointegração e entra em $\alpha_{1}, \alpha_{2}$ e $\alpha_{3}$, ou seja, a relação de PPC, 
sem restrições sobre as taxas de juros, entra 3 vezes. A estatística obtida tem distribuição $\chi^{2}(6)$, e neste caso assume um valor de 43,53. É um valor alto, e a hipótese nula é rejeitada confortavelmente.

b) $H_{0}$ : O vetor $\left[\begin{array}{lllll}1 & -1 & -1 & 0 & 0\end{array}\right]$ pertence ao espaço de cointegração. A estatística obtida tem distribuição $\chi^{2}(2)$, e neste caso assume um valor de 3,03, com um p-value de 0,22. A hipótese nula não é rejeitada, à um nível de significância de 0,10. Em outras palavras, a hipótese de que a relação PPC é estacionária não é rejeitada. Neste teste, temos feito entrar a relação PPC uma vez e não 3 vezes com em a).

c) $\mathrm{H}_{\mathrm{o}}$ : $\mathrm{O}$ vetor $\left[\begin{array}{lllll}0 & 0 & 0 & 1 & -1\end{array}\right]$ pertence ao espaço de cointegração. A estatística obtida tem distribuição $\chi^{2}(2)$, e neste caso assume o valor de 8,93. A hipótese nula não é rejeitada ao nível de significância de 0,01. Em outras palavras, a hipótese de que a relação 2, o diferencial das taxas de juros (jurfr-tbill), é estacionária não é rejeitada, embora por escassa margem.

Note que se os vetores em b) e c) pertencem ao espaço de co-integração, a relação 4 (ipafr-ipaus-ffus-jurfr+tbill) também pertence ao espaço de co-integração.

Vale a pena lembrar que o resultado em cl já havia sido antecipado pelo teste de raízes unitárias de Dickey-Fuller na seção 7.2.1. Entretanto, a hipótese nula de raíz unitária para a PPC não tinha sido rejeitada. Isto pode ser explicado em parte pelo fato de que os testes de raízes unitárias feitas na seção 7.2.1 não levam em conta as outras variáveis do modelo, contrariamente aos testes de Johansen.

d) $H_{0}$ : $O$ vetor $\left[\begin{array}{lllll}0 & 0 & 1 & 1 & -1\end{array}\right]$ pertence ao espaço de cointegração. A estatística obtida tem distribuição $\chi^{2}(2)$, e neste caso assume o valor de 6,18. A hipótese nula não é rejeitada ao nível de significância de 0,05. Em outras palavras, o dólar guarda uma relação diretamente proporcional com o diferencial de taxas de juros EUA-França (relação 3, ffustjurfr-tbill), e esta relação é estacionária, de acordo ao que sugere o resultado do teste (resultado diferente ao obtido pelo teste de Dickey Fuller). Neste caso, é interessante apresentar o $\alpha^{\prime}$ estimado no modelo restrito. 


\section{Quadro 7.10}

\begin{tabular}{rrr}
\multicolumn{3}{c}{$\alpha$} \\
$\alpha_{1}$ & $\alpha_{2}$ & \multicolumn{1}{c}{$\alpha_{3}$} \\
& & \\
0,000 & 0,404 & $-0,990$ \\
0,000 & $-0,486$ & 0,946 \\
1,000 & $-0,468$ & 1,146 \\
1,000 & 1,000 & 1,000 \\
$-1,000$ & 0,532 & 2,146
\end{tabular}

Note como a relação PPC aparece na segunda e na terceira colunas de forma bem pronunciada.

Considerando alguns dos resutados no modelo de Tendências Comuns que serão tratados mais adiante, foram feitos dois testes adicionais.

e) $H_{0}$ : $O$ vetor $\left[\begin{array}{lllll}a & 0 & 0 & -b & 0\end{array}\right]$ pertence ao espaço de cointegração. A estatística obtida tem distribuição $\chi^{2}(1)$, e neste caso assume o valor de 0,47. A hipótese nula não é rejeitada confortavelmente. Este resultado sugere a existência de uma relação de longo prazo entre as taxas de juros e os preços na França.

f) $H_{0}$ : O vetor $\left[\begin{array}{lllll}0 & 0 & a & 0 & -b\end{array}\right]$ pertence ao espaço de cointegração. A estatística obtida tem distribuição $\chi^{2}(1)$, e neste caso assume o valor de 2,86. A hipótese nula não é rejeitada confortavelmente. Este resultado sugere que o dólar aumenta (cai) quando aumentam (caem) as taxas de juros nos EUA, e que esta relação é estacionária.

g) Finalmente foi testada a exogeneidade fraca dos preços da França, das taxas de juros nos USA e da França. Os testes obtidos, que tem distribuição $\chi^{2}(5)$, foram respectivamente 40,$1 ; 24,24$ e 18,94. Exogeneidade fraca destas variáveis foi rejeitada confortavelmente.

Antes de continuar com o cálculo das outras representações, vale apresentar as seguintes observações. As hipóteses nulas de que os vetores $\left[\begin{array}{lllll}1 & -1 & -1 & 0 & 0\end{array}\right]$ (pela teoria da PPC) e $\left[\begin{array}{lllll}0 & 0 & 0 & 1 & -1\end{array}\right]$ (pelo diferencial das taxas de juros implicado pela teoria da PJNC) pertencem ao espaço de cointegração, não foram rejeitadas, embora por escasa margem. Os resultados relativos à $P P C$ neste sentido (ausência de forte evidência estatística em favor a esta teoria) se assemelhan aos resultados obtidos em outros estudos para taxa de câmbio de moedas européias com relação ao dólar para - mesmo período, que é (em sua maioria) a era pos-Bretton Woods. Hali J. Edison et alli. (1994) menciona uma série de causas sobre o porqué destes resultados :

(i) O período considerado (algo mais de 20 anos) é relativamente curto. Estudos feitos para periodos mais longos proporcionan evidência 
mais robusta em favor da PPC (Edison \& Klowland, 1987; Ardeni \& Lubian, 1991 e Kim, 1990).

(ii) Os autores notam que muitos trabalhos claman sobre que a relação PPC passa os testes de estacionariedade, mas de fato estes trabalhos falam sobre uma relação entre a taxa de câmbio e preços com os sinais apropriados para a PPC, mas não assumen a relação de proporcionalidade e simetria implicada, i.e. que o vetor de co-integração é exatamente $\left[\begin{array}{lllll}1 & -1 & -1 & 0 & 0\end{array}\right]$.

(iii) O trabalho dos autores documenta em particular sobre experimentos Montecarlo feitos para os testes de Johansen sobre cointegração. Nestes estudos, os testes revelam ter um baixo poder para amostras pequenas : em particular nos testes com as estatísticas do Traço e Lambdamax, o poder quando a hipótese nula é falsa é práticamente igual ao tamanho dos testes, i.e. a probabilidade de rejeitar não co-integração quando existe co-integração é práticamente a mesma que a probabilidade de rejeitar não co-integração quando não tem co-integração. Note que no presente trabalho, apesar disto, o teste de Johansen admitiu um espaço de co-integração de dimensão 3, dentro de um VAR de dimensão 5, que inclui não somente preços e taxa de câmbio, mas também taxas de juros e variáveis exógenas. Os autores se referem à fraca evidência em favor da PPC dentro de modelos que consideram somente preços e taxa de câmbio. Provavelmente o modelo VAR(5) que inclui também taxas de juros e variáveis exógenas, esteja mais perto do Mecanismo Estatístico Gerador Correto, o que ajuda a admitir o espaço de co-integração sugerido pela teoria.

(iv) Os autores falam também, para o caso dos testes de Johansen, sobre a obtenção de uma correção dos valores críticos levando em conta o tamanho da amostra e, mais importante, o tamanho do período cosiderado. Mesmo assim, eles documentam sobre o fraco poder dos testes (embora mais eficazes que os testes de Dickey-Fuller), sugerindo técnicas alternativas para testar co-integração.

Nas próximas seções serão calculadas ${ }^{4}$ as representações VAR Restrita e de Wold, para finalmente apresentar o Modelo de Tendências Comuns, baseados na hipótese de um espaço de co-integração de dimensão 3, o que implica a presença de duas tendências comuns.

\subsubsection{A Representação VAR Restrita}

Uma vez estimada a equação (7.10), e com a hipótese de que o espaço de co-integração é de dimensão $3(r=3)$, implicando a existência de 2 tendências comuns $(k=2)$ podemos calcular a representação VAR restrita, discutida no capítulo 3 :

$$
\mathrm{B}(\mathrm{L}) \mathrm{Y}_{\mathrm{t}}=\theta+\sum_{i} \theta_{\mathrm{i}}^{*} \mathrm{D}_{\text {it }}+\sum_{j} \theta_{j}^{*} Z_{j t}+\eta_{t}
$$

\footnotetext{
${ }^{4}$ Os procedimentos a seguir foram realizados usando o programa elaborado por E. Melander, que funciona como um procedimento dentro do programa "RATS"
} 
onde :

$$
\begin{gathered}
B(L)=M\left[A^{*}\left(L_{)} M^{-1} D(L)+\gamma^{*} L\right]\right. \\
y_{t}^{*}=D_{\perp}(L) N X_{t}
\end{gathered}
$$

e

$$
\theta=\mathrm{M} \rho, \quad \theta_{i 1}{ }^{*}=\mathrm{M} \rho_{i}{ }^{*}, \quad \theta_{j 2}{ }^{*}=\mathrm{M} \omega_{j}, \quad \eta_{\mathrm{t}}=\mathrm{M} \varepsilon_{\mathrm{t}}
$$

com a matriz de dimensão $5 \times 5 \mathrm{M}=\left[\mathrm{S}_{2}{ }^{\prime} \alpha\right]^{\prime}$, sendo que $\mathrm{S}_{2}$ é de dimensão $2 \times 5$ e é ortogonal a $\alpha . \quad \gamma^{\star}=\left[\begin{array}{ll}0 & \gamma\end{array}\right]$ é uma matriz de dimensão $5 \times 5$ e $D(L)$ e $D_{1}(L)$ são matrizes polinomiais, de dimensão $5 \times 5$ :

$$
\mathrm{D}(\mathrm{L})=\left[\begin{array}{cc}
\mathrm{I}_{2} & 0 \\
0 & \left(1-\mathrm{L}_{)} \mathrm{I}_{3}\right.
\end{array}\right] \quad \text { e } \quad \mathrm{D}_{1}(\mathrm{~L})=\left[\begin{array}{cc}
\left(1-\mathrm{L}_{1} \mathrm{I}_{2}\right. & 0 \\
0 & \mathrm{I}_{3}
\end{array}\right]
$$

A matriz $\mathrm{M}$ calculada, após 0 cálculo da matriz $\mathrm{S}_{2}$ ortogonal a $\alpha$ é :

\section{Quadro 7.11}

Matriz M

$\begin{array}{cccll}0,4097404 & 0,6674019 & -0,61955+1 & 0,03269814 & -0,04208298 \\ 0,6390460 & 0,2751794 & 0,7133930 & -0,223+167 \mathrm{E}-15 & 0,08346860 \\ 1,000000 & -1,228000 & -0,6010000 & 3,114000 & 1,529000 \\ 1,088000 & -1,109000 & -0,250(0) 000 & 1,0000000 & -2,537000 \\ -0,03400000 & 0,0190000 & 0,08900000 & 1,000000 & -0,5630000\end{array}$

Usando (7.13), (7.15) e as estimativas de (7.10), são calc9ulados os parâmetros da representação VAR Restrita. As matrizes $B_{1}$ e $B_{2}$ do polinômio $B(L)$ são apresentados a seguir :

\section{Quadro 7.12}

Matriz $\mathrm{B}_{1}$

$\begin{array}{lllcc}0,9091301 & 0,1084726 & -0,2315630 & -0,30+2876 & 10,366805 \\ -0,5234745 & 0,05825589 & 0,2314965 & 0,139+906 & -10,326545 \\ 10,396040 & 0,4507312 & 0,6725006 & -0,3461654 & 10,649657 \\ -0,06675792 & 0,03929458 & 0,02+20824 & 0,9476783 & -0,2115357 \\ -0,20938420 & 0,01569174 & 0,08512052 & 0,05555334 & 0,7586930\end{array}$


Quadro 7.12 (continuação)

Matriz $\mathrm{B}_{2}$

$\begin{array}{ccccc}0,0000000 & 0,0000000 & 0,1586777 & 0,3044716 & -1,350427 \\ 0,0000000 & 0,0000000 & -0,1532893 & -0,2810035 & 1,100834 \\ 0,0000000 & 0,0000000 & 0,039278360 & 0,3211324 & -2,134454 \\ 0,0000000 & 0,0000000 & -0,075487240 & -0,046084350 & 0,2595697 \\ 0,0000000 & 0,0000000 & -0,073441520 & -0,049388340 & 0,1321920\end{array}$

As estimativas dos parâmetros da constante, e das variáveis sazonais são apresentados no Quadro 7.13 :

\section{Quadro 7.13}

Variáveis Sazonais e Constante

$\begin{array}{cccc}0_{11}{ }^{*} & 0_{21}{ }^{*} & \theta_{31}{ }^{*} & \theta \\ -0,01157052 & -0,01163448 & -0,007194884 & -0,1356998 \\ 0,001098215 & 0,01671580 & 0,004045631 & 0,07498398 \\ -0,02002600 & -0,01619000 & -0,001294000 & -0,5004000 \\ -0,01098400 & -0,00861300 & -0,007032000 & -0,1798740 \\ 0,00086300 & -0,00350100 & -0,000384000 & 0,04456500\end{array}$

As estimativas dos parâmetros correspondentes as outras variáveis exógenas são apresentadas no quadro 7.14 :

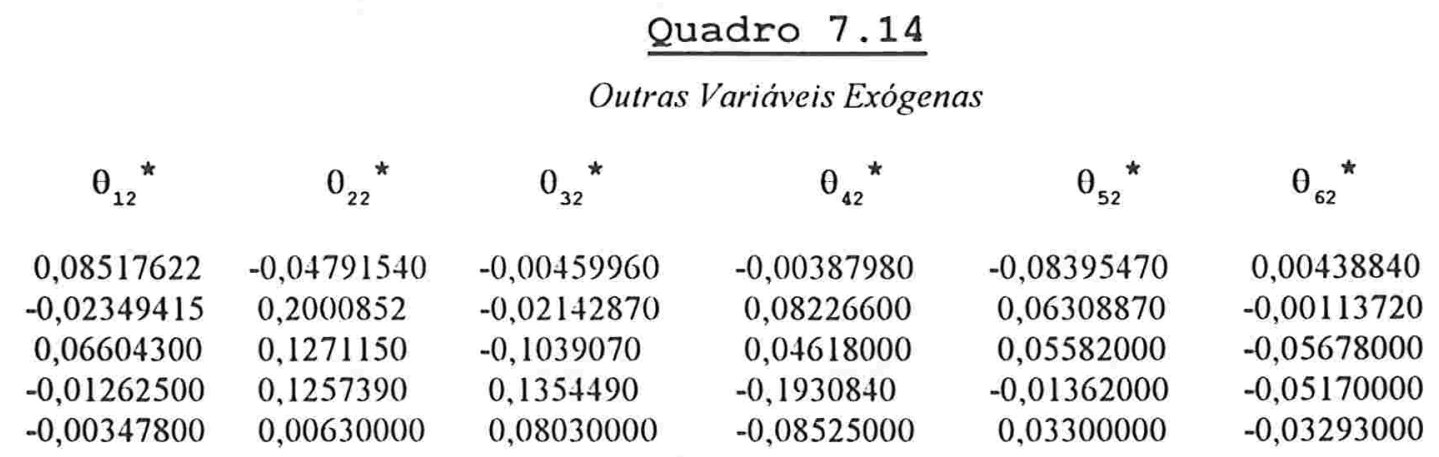

\subsubsection{A Representação de Wold}

A partir da representação VAR restrita podemos obter a representação de Wold, de acordo à descrição do procedimento no capítulo 4 : 


$$
\Delta x_{t}=\delta+C(L)\left[\rho_{1}^{*} D_{1 t}+\rho_{2}^{*} D_{2 t}+\rho_{3}^{*} D_{3 t}+\sum_{j} \omega_{j} Z_{j t}+\varepsilon_{t}\right]
$$

onde :

$$
C(L)=M^{-1} D(L) B(L)^{-1} M=\sum_{i=0}^{\infty} C_{i} L^{i} \quad \text { e } \quad \delta=C(1) \rho
$$

A seguir são apresentadas as estimativas de $C(1)$ e $\delta$ :

\begin{tabular}{clcll}
\multicolumn{5}{c}{ Quadro 7.15} \\
\cline { 3 - 4 } Matriz C $(1)$ \\
$-0,05674773$ & 2,896961 & $-0,3884676$ & $-2,748742$ & 3,449009 \\
0,1803050 & 1,931980 & $-0,4724782$ & $-0,7895033$ & 0,5840096 \\
$-0,5124640$ & 0,732450 & 0,2358164 & $-3,933088$ & 5,827043 \\
0,013635740 & 0,04558901 & $-0,02032631$ & 0,02624912 & $-0,06001764$ \\
$-0,04727947$ & 0,2450942 & 0,008689451 & $-0,4357709$ & 0,6259664 \\
& \multicolumn{5}{c}{ Vetor } & $\delta^{\prime}$ \\
$-0,1356998$ & 0,07498398 & $-0,5004000$ & $-0,1798740$ & 0,04456500
\end{tabular}

7.2.7 Identificação dos Parâmetros da Representação de Tendências Comuns Comuns :

O processo em estudo admite a representação de Tendências

$$
\begin{gathered}
\chi_{t}=\chi_{0}+K \tau_{t}+\Phi(L)\left[v_{t}+\sum_{i} \Psi_{i j} D_{i t}+\sum_{j} \Psi_{j} Z_{j t}\right] \\
\tau_{t}=\mu+\sum_{i} \mu_{i 1}^{*} D_{i \pm}+\sum_{j} \mu_{j}^{*} Z_{j}+\tau_{t-1}+\varphi_{t}
\end{gathered}
$$

onde :

(i) $\chi_{0}$ é o vetor constante contendo os valores iniciais de $x_{t}$. 
(ii) $\mathbf{K}$ é uma matriz de constantes de dimensão $5 \times 2$, sendo que 2 é o número de tendências, $\tau_{t}$ é um vetor de dimensão $2 \times 1$, a variável de tendência. A seqüência 5-dimensional $\left\{\boldsymbol{v}_{t}\right\}$ é um ruído branco com $E\left(v_{t}\right)=0$ e $E\left(v_{t} v_{t}^{\prime}\right)=I_{5}$ a matriz identidade $5 \times 5$.

(iii) Os vetores de parâmetros $\Psi_{i 1}, \Psi_{j 2}$ são de dimensão $5 \times 1$; os vetores de parâmetros $\mu, \mu_{i 1}{ }^{*}$ e $\mu_{j 2}{ }^{*}$ são vetores de dimensão $2 \times 1 .\left\{\varphi_{t}\right\}$ é uma sequiência ruído branco $\operatorname{com} E\left(\varphi_{t}\right)=0$ e $E\left(\varphi_{t} \varphi_{t}^{\prime}\right)=I_{2}$.

Cálculo dos parâmetros do componente permanente. Para o cálculo da matriz K, será usado o procedimento 4) descrito no capítulo 5, implicando o cálculo das matrizes $\mathbf{K}_{0}$ e $\pi$. $\mathbf{K}_{0}$ é uma matriz de dimensão $5 \times 2$ e posto 2 , que exige a observação da restrição $\alpha^{\prime} \mathbf{K}_{0}=0$. $\pi$, de dimensão $2 \times 2$, é tal que $\mathbf{K}_{0} \pi=\mathbf{K}$, e é obtida usando a restrição $\mathbf{K}_{0} \pi \pi^{\prime} \mathbf{K}_{0}{ }^{\prime}=\mathrm{C}(1) \Sigma C(1)^{\prime}$, o que resulta em uma decomposição de Choleski da matriz $\left[\mathbf{K}_{0}^{\prime} \mathbf{K}_{0}\right]^{-1} \mathbf{K}_{0}^{\prime} \mathbf{C}(\mathbf{l}) \Sigma \mathrm{C}\left(l^{\prime} \mathbf{K}_{0}\left[\mathbf{K}_{0}^{\prime} \mathbf{K}_{0}\right]^{-1}\right.$. As matrizes $\mathbf{K}_{0}$ e $\pi$ são apresentadas a seguir :

$\begin{array}{cc}\frac{\text { Quadro }}{2.16} \\ \text { Matriz } \mathrm{K}_{\mathbf{0}} \\ 0,4097404 & 0,6390460 \\ 0,6674019 & 0,2751794 \\ -0,6195541 & 0,7133930 \\ 0,03269814 & -0,2234167 \text { E-15 } \\ -0,04208298 & 0,08346860\end{array}$

Matriz $\pi$

$\begin{array}{cc}0,03164780 & 0,0000000 \\ -0,007182451 & 0,06462318\end{array}$

Assim pode-se calcular a matriz $5 \times 2 \mathrm{~K}$ em (7.19) : 


\section{Quadro 7.17}

Matriz K

$\begin{array}{ll}0,008377468 & 0,04129718 \\ 0,01914534 & 0,01778297 \\ -0,02473144 & 0,04610172 \\ 0,001034824 & -0,1443790 \mathrm{E}-16 \\ -0,001931343 & 0,005394006\end{array}$

Note que a matriz $\mathbf{K}$ não é única, pois $\pi$ não é única, o que decorre da natureza da própria decomposição de Choleski.

Os outros parâmetros do componente permanente da representação de Tendências Comuns são obtidos usando as restrições :

$$
\begin{aligned}
& \mu=\left(K^{\prime} K\right)^{-1} K^{\prime} C(l) M^{-1} \theta \\
& \mu_{\dot{i}}^{*}=\left(K^{\prime} K\right)^{-1} K^{\prime} C(l) M^{-1} \theta_{\dot{i}}^{*} \\
& \mu_{\ddot{j}}^{*}=\left(K^{\prime} K\right)^{-1} K^{\prime} C(l) M^{-1} \theta_{\dot{j}}^{*}
\end{aligned}
$$

Os parâmetros estimados são :

$$
\text { Quadro } 7.18
$$

Coeficientes das Variáveis Sazonais e Constante no Componente Permanente

$$
\begin{array}{rrcc}
\mu_{11} * & \mu_{21} * & \mu_{31} * & \mu \\
-0,3588088 & -0,6001886 & -0,3872810 & 0,3848939 \\
-0,1060638 & 0,2864037 & 0,03183866 & 0,1940285 \\
& \text { Quadro } & 7.19 &
\end{array}
$$

Coeficientes das Outras Variáveis Exógenas no Componente Permanente

$\begin{array}{cccccc}\boldsymbol{\mu}_{12}{ }^{*} & \mu_{22}{ }^{*} & \mu_{32}{ }^{*} & \mu_{42}{ }^{*} & \mu_{52}{ }^{*} & \mu_{62}{ }^{*} \\ 3,289636 & -1,173347 & 3,228476 & -5,352240 & -0,9642828 & -0,5910509 \\ 3,150522 & -0,6574102 & -6,261312 & 5,428084 & -2,273984 & 2,833011\end{array}$

Cálculo dos parâmetros do componente transitório. Por último, serão calculados os parâmetros do componente transitório na equação (7.19), o que permite o estudo do efeito de choques nas variáveis do sistema na representação 
de Tendências Comuns. Devemos partir da Representação de Wold em (7.17), escrita da seguinte forma :

$$
\Delta \chi_{t}=\delta+C(L) F^{-1}\left[F \sum_{i} \rho_{i}^{*} D_{i \pm}+F \sum_{j} \omega_{j} Z_{j t}+F \varepsilon_{t}\right]
$$

onde F é uma matriz não singular tal que :

$$
v_{\mathrm{t}}=\left[\begin{array}{l}
\varphi_{\mathrm{t}} \\
\psi_{\mathrm{t}}
\end{array}\right]=\mathrm{F}_{\mathrm{t}} \quad \mathrm{F}:=\left[\begin{array}{l}
\mathrm{F}_{2} \\
\mathrm{~F}_{3}
\end{array}\right] \quad \therefore \quad\left[\begin{array}{l}
\varphi_{\mathrm{t}} \\
\psi_{\mathrm{t}}
\end{array}\right]=\left[\begin{array}{l}
\mathrm{F}_{2} \\
\mathrm{~F}_{3}
\end{array}\right] \varepsilon_{\mathrm{t}}
$$

Vale lembrar que $v_{t}$ são os choques que se produzem no componente transitório dentro da representação de Tendências Comuns (7.19). Temos estabelecido a restrição de que $v_{t}$ é função de $\varphi_{t}$, processo (de dimensão $2 \times 1$ ) que representa os choques no componente permanente, sendo que $\psi_{t}$ (de dimensão $3 \times 1)$ são choques no componente transitório independentes dos choques que se produzem no componente permanente. Assim, são obtidos os coeficientes das varíveis exógenas no componente transitório :

$$
\Psi_{\mathrm{i}}=F \mathrm{p}_{i}^{*} \quad \Psi_{j}=F \omega_{j}
$$

e definimos :

$$
R(L)=C(L) F^{-1}
$$

Obtemos assim a equação onde serão aplicadas as inovações :

$$
\Delta \chi_{t}=\delta+R(L)\left[\sum_{i} \Psi_{\dot{j}} D_{i \pm t}+\sum_{j} \Psi_{j p} Z_{j t}+v_{t}\right]
$$

F é obtida das equações:

$$
\begin{aligned}
& F_{2}=\left(K^{\prime} K\right)^{-1} K^{\prime} C(l) \\
& F_{3}=Q^{-1} \gamma \Sigma^{-1}
\end{aligned}
$$


onde a matriz $Q$, de dimensão $r \times r$, neste caso $3 \times 3$, pode ser escolhida de modo que a matriz de covariâncias das inovações transitórias $\mathrm{E}\left[\boldsymbol{\psi}_{\mathrm{t}} \boldsymbol{\psi}^{\prime}{ }_{\mathrm{t}}\right]$ seja diagonal.

As matrizes $Q$ e $F$ estimadas são :

\begin{tabular}{cccc}
\multicolumn{4}{c}{ Quadro 7.20} \\
& Matriz Q \\
224,5646 & 0,000000 & 0,00000 & \\
59,77245 & 208,918900 & 0,00000 & \\
$-19,10952$ & $-3,136275$ & 55,16565 & \\
& & & \\
& Matriz F & & \\
43,93476 & $-19,65642$ & 24,98040 & $-57,92067$ \\
61,93153 & $-5,361871$ & $-70,23232$ & 95,30614 \\
$-7,281366$ & $-2,521194$ & 115,3315 & 55,44085 \\
39,0924 & 12,65964 & 47,97481 & $-55,07968$ \\
4,316706 & $-0,3679074$ & $-4,922752$ & 6,689059
\end{tabular}

As estimativas dos coeficientes das variáveis sazonais e das variáveis exógenas no componente transitório são :

\section{Quadro 7.21}

Coeficientes das Variáveis Sazonais no Componente Transitório

$\begin{array}{ccc}\Psi_{11} * & \Psi_{21} * & \Psi_{31} * \\ -0,5490570 & 2,949772 & 0,1791749 \\ 1,92030 & -1,955690 & 0,3739215 \\ -0,6105757 & -3,168733 & -1,027257 \\ -1,103924 & 3,922221 & 0,4808905 \\ -0,503588 & -0,7804599 & -0,3709476\end{array}$

Quadro 7.22

Coeficientes das Outras Variáveis Exógenas no Componente Transitório

$\begin{array}{cccccc}\boldsymbol{\Psi}_{\mathbf{1 2}}{ }^{*} & \Psi_{\mathbf{2 2}}{ }^{*} & \Psi_{\mathbf{3 2}}{ }^{*} & \Psi_{\mathbf{4 2}}{ }^{*} & \Psi_{52}{ }^{*} & \Psi_{\mathbf{6 2}}{ }^{*} \\ 0,7564973 & 7,642800 & -20,73953 & 14,68010 & -29,15391 & 22,12933 \\ -5,006970 & 5,559101 & 29,65422 & -13,68300 & 49,75718 & -32,27410 \\ -4,176815 & 14,34460 & 39,76550 & -39,94881 & 24,27923 & -25,62262 \\ -1,313570 & 34,03541 & -22,69781 & 15,50693 & -21,47984 & 16,18111 \\ 4,348951 & -0,4401609 & 1,679227 & -4,295735 & 0,8218711 & -1,905240\end{array}$

A matriz polinomial infinita $R(L)$ não é apresentada aqui. 
O gráfico 7.4 exibe as duas tendências $\left(\tau_{t}\right)$ estimadas. Nos gráficos 7.5 e 7.6 são apresentados os componentes permanente e transitório calculados das variáveis do modelo.

\section{Gráfico 7.4}

\section{Tendências Comuns}
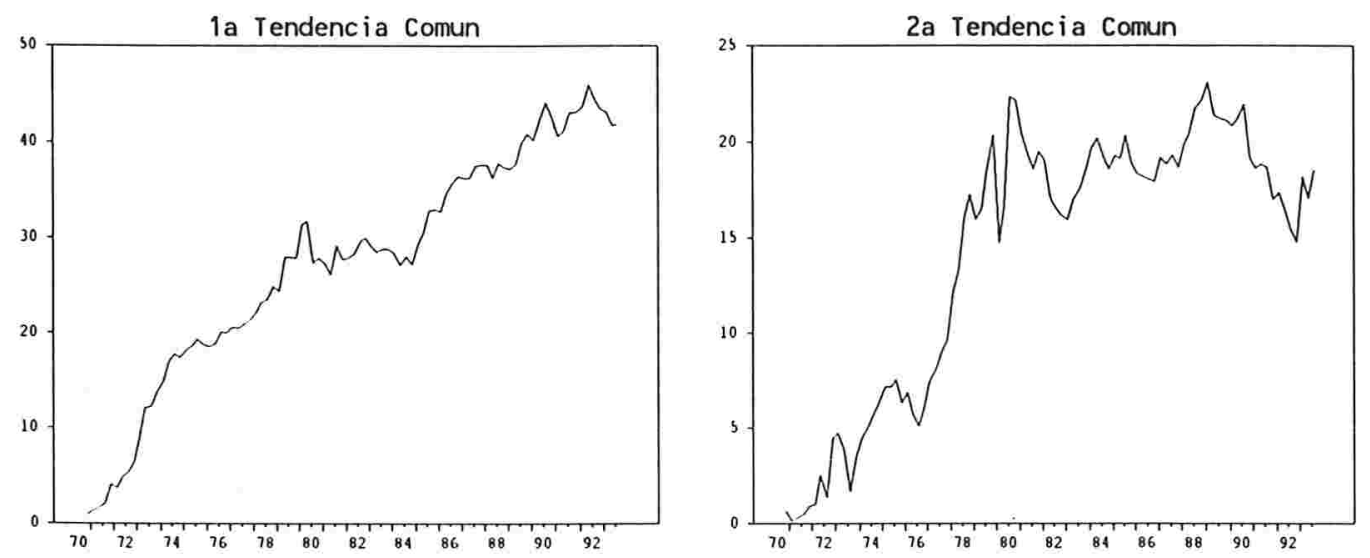

A comparação dos gráficos 7.5 e 7.4 sugere que as variáveis ipafr, ipaus e jurfr estão dominadas pela $1^{a}$ tendência, sendo que ffus e tbill seguem predominantemente a $2^{a}$ tendência. Uma observação mais cuidadosa, no entanto, revela que ipafr recebe uma influência maior da $2^{a}$ tendência. No gráfico 7.5, chama a atencão, por um lado, a semelhança dos gráficos de tbill e ffus, e, por outro lado, o componente permanente de jurfr, com tendência marcadamente crescente, contrariamente a tbill (com base nestes gráficos foram feitos os testes adicionais de co-integração de ipafr com jurfr, e ffus com tbill, testes e) e f) na seção 7.2.3).

Em outras palavras, a análise gráfica sugere a presença de uma tendência que parece estar em relação com o crescimento secular dos preços, embora ela influencie também as taxas de juros na França. A outra tendência parece estar predominantemente associada ao mercado de câmbio e às taxas de juros nos EUA, embora ela influencia também os preços da França.

O exame das tendências não é de fato muito conclusivo. Vale a pena mencionar de novo que a matriz $\mathbf{K}$ não é exatamente identificada, dentro do método que temos utilizado aqui. Assim, a tendência $\tau_{t}$, que é calculada partindo do componente permanente $\mathrm{K} \tau_{\mathrm{t}}$, também não é exatamente 
identificada. Em outras palavras, uma outra matriz $\mathbf{K}$ terianos conduzido a tendências diferentes das expostas aqui.

\subsubsection{Cálculo de Respostas de Impulsos Associados a Choques nos Componentes Permanente e Transitório}

Foi efetuado o cálculo de respostas de impulsos para o sistema definido na equação (7.22) aplicando choques de um desvio padrão. Como a matriz de variância-covariância $E\left(\boldsymbol{v}_{\mathbf{t}} \boldsymbol{v}_{\mathbf{t}}^{\prime}\right)$ é uma matriz identidade, foram então aplicados choques de tamanho 1.

O gráfico 7.7 exibe respostas de impulsos em $\Delta \chi_{t^{\prime}}$ estimados para 20 trimestres avançados, para inovações no componente permanente. Tem dois quadros por variável, sendo que o número de tendências é dois : o efeito de um choque em cada uma das tendências sobre cada uma das variáveis é analisado. Por exemplo, os dois primeiros quadros do gráfico 7.7 apresentam a resposta da variável ipafr (índice de preços no atacado na França) a choques no componente permanente. Pode-se ver que ainda após 20 períodos, a variável ipafr não tem atingido seu equilíbrio de longo prazo. Um comportamento semelhante é observado em ipaus (índice de preços no atacado nos EUA). As variáveis jurfr e tbill (taxas de juros na França e nos EUA) e ffus (taxa de câmbio FFr/US\$) atingem mais rapidamente o equilíbrio, em torno de 10 trimestres.

O gráfico 7.8 exibe respostas de impulsos associados a inovações no componente transitório. Em geral, o equilíbrio é atingido mais rapidamente que no caso de choques no componente permanente.

Vale a pena aqui lembrar o seguinte. Normalmente na representação de médias móveis de um processo multivariado :

$$
y_{t}=\sum_{i=0}^{\infty} \Phi_{i} u_{t-i}
$$

a matriz $\Sigma_{\mathbf{u}}=\mathrm{E}\left(\mathrm{u}_{\mathrm{t}} \mathrm{u}^{\prime}{ }_{t}\right)$ não é diagonal. Na análise de resposta de impulsos, que usualmente é feita sobre a representação de médias móveis, aplica-se uma inovação a uma variável, forçando às outras inovações a assumir o valor 0 . Dada a natureza de $\Sigma_{\mathbf{u}}$, choques a uma variável do sistema devem, pelo contrário, afetar às outras variáveis do sistema no mesmo instante, de modo que a análise de respostas de 
impulsos no contexto de (7.28) de fato está obscurecendo a reação real do sistema.

Então, a análise de respostas de impulsos é feita numa representação alternativa :

$$
y_{t}=\sum_{i=0}^{\infty} R_{i} v_{t-i}
$$

que é obtida fazendo a decomposição $\Sigma_{\mathrm{u}}=\mathrm{PP}^{\prime}$ onde $\mathrm{P}$ é uma matriz triangular inferior. Assim, definimos $v_{t}=\mathrm{P}^{-\mathbf{1}_{\mathbf{u}_{t}}}$, de modo que $\Sigma_{v}=E\left(v_{t} v^{\prime}{ }_{t}\right)=E\left(P^{-1} u_{t} u_{t}{ }^{\prime} P^{-1 \prime}\right)=I_{n}$ e $R_{i}=\Phi_{i} P$. Assim, uma mudança em um dos componentes de $v_{t}$ não afetará os outros componentes de $v_{t}$.

O procedimento descrito no parágrafo acima é o que foi feito neste trabalho. Ora, a escolha da matriz P não é única e um diferente ordenamento das variáveis alterarão $P, e$, em consequência os coeficientes $R_{i}$, mudando o perfil da função de respostas de impulso. A extensão destas mudanças dependerá do grau de correlação existente entre os componentes de $u_{t}$.

Portanto, toda conclusão relativa às respostas de impulso do sistema deve ser feita levando em consideração a discussão no parágrafo acima. 


\section{Gráfico 7.5}

\section{Componentes Permanentes Estimados}
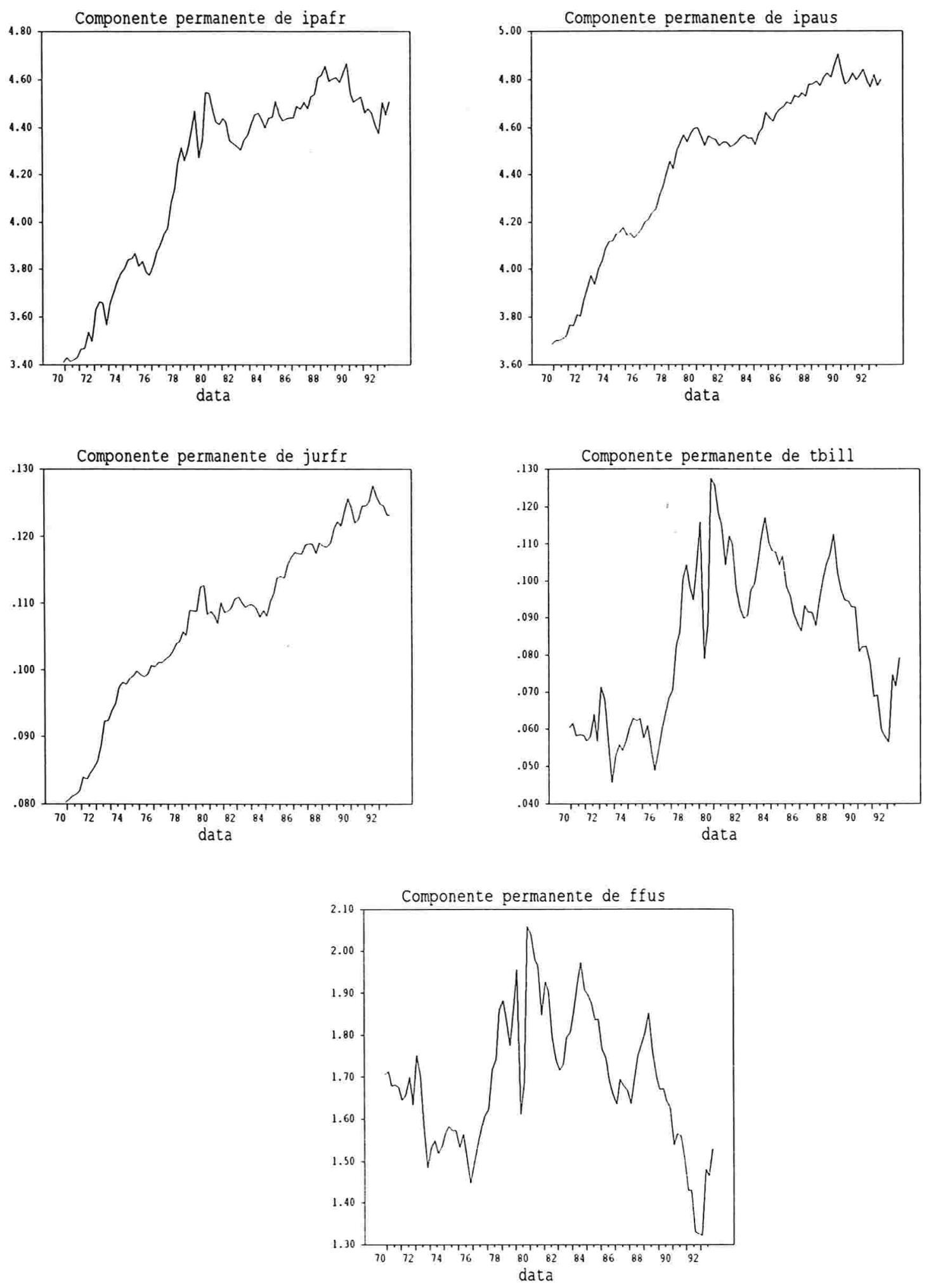


\section{Gráfico 7.6}

\section{Componentes Transitórios Estimados}
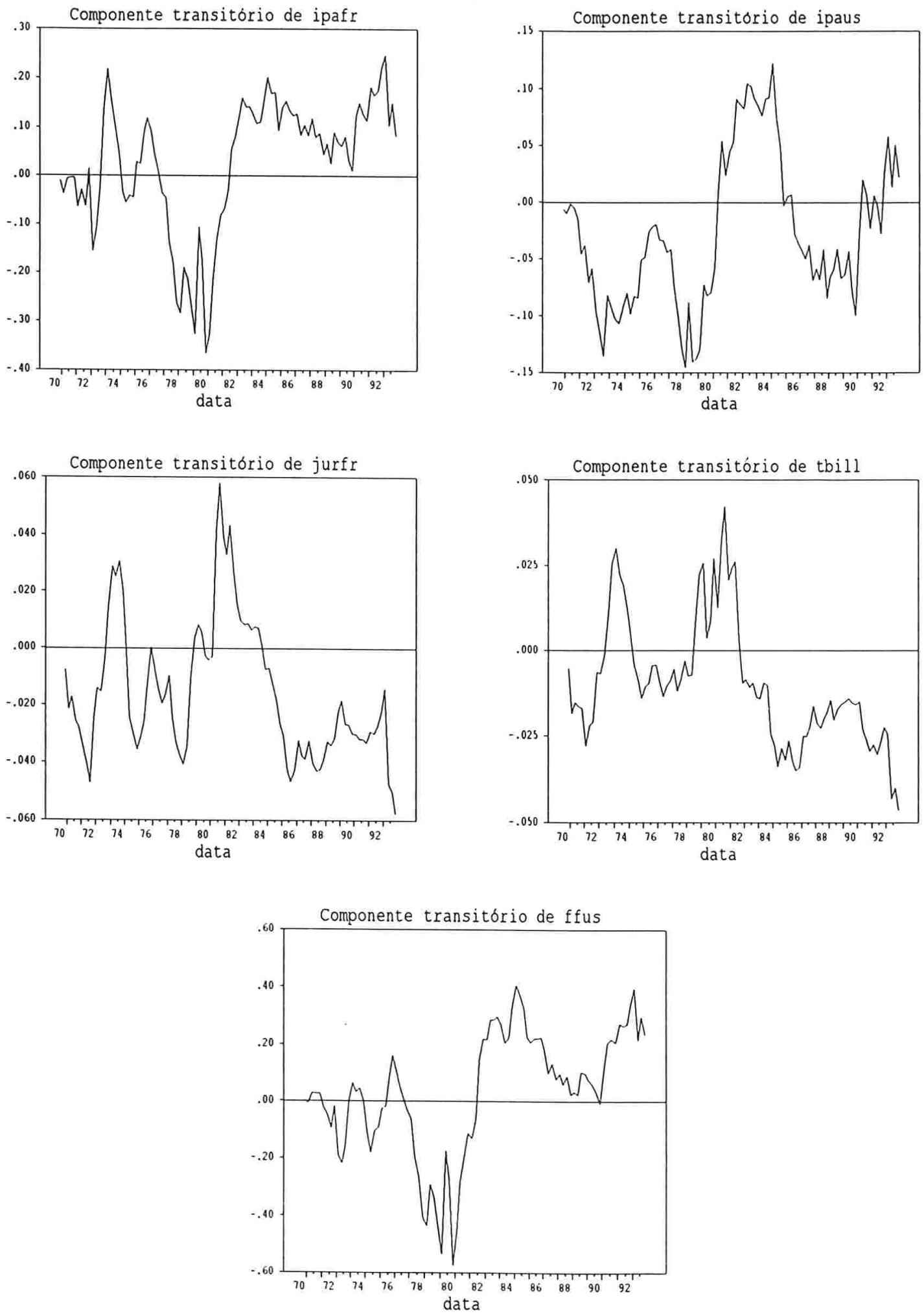


\section{Gráfico 7.7}

Respostas de Impulsos Estimados de $\Delta \chi_{t}$ para Inovações no Componente Permanente
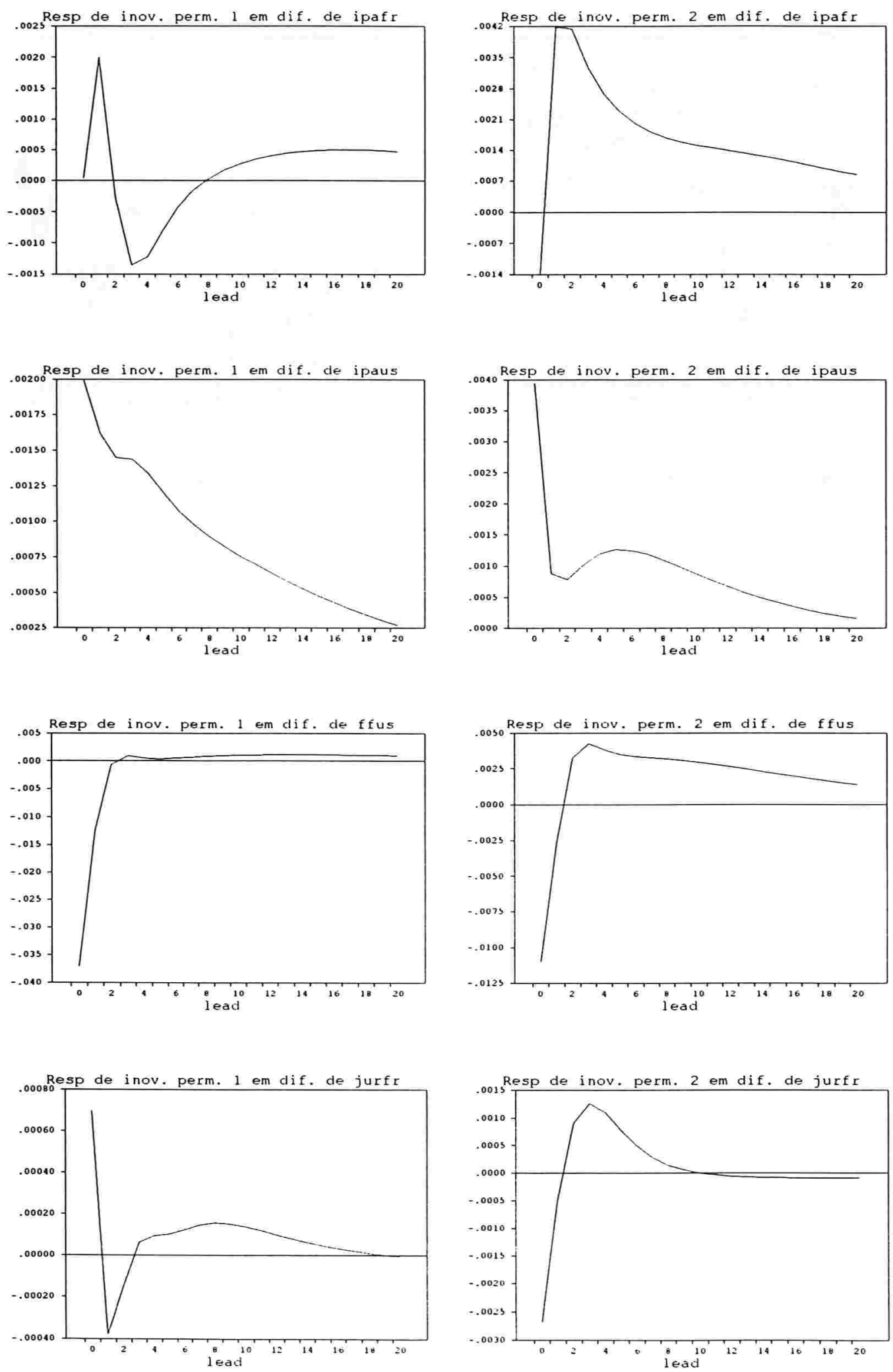


\section{Gráfico 7.7 (continuação)}

Respostas de Impulsos Estimados de $\Delta \chi_{t}$ para Inovações no Componente Permanente
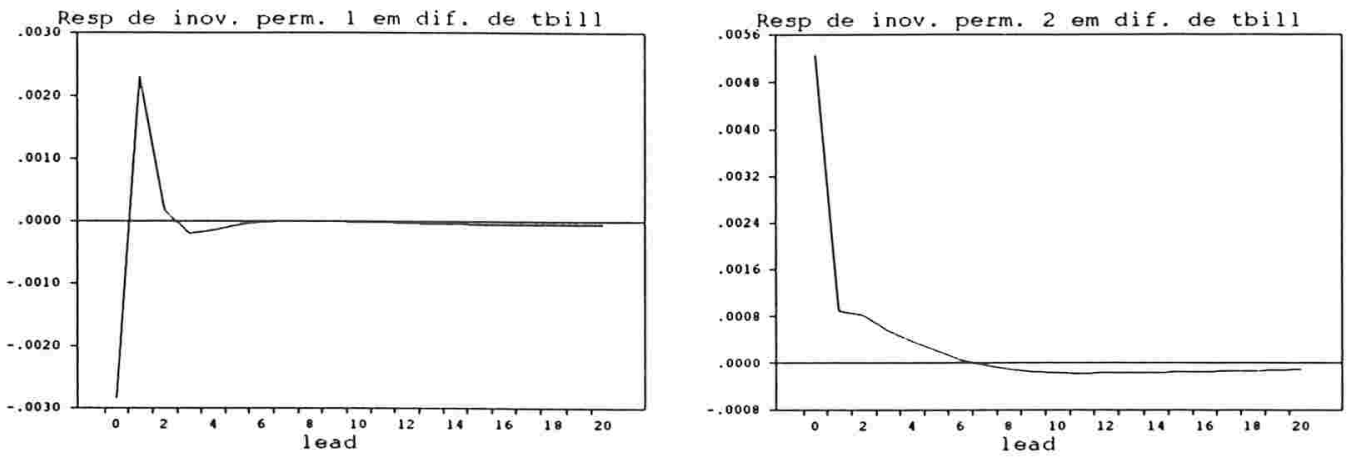

\section{Gráfico 7.8}

Respostas de Impulsos Estimados de $\Delta \chi_{t}$ para Inovações no Componente Transitório
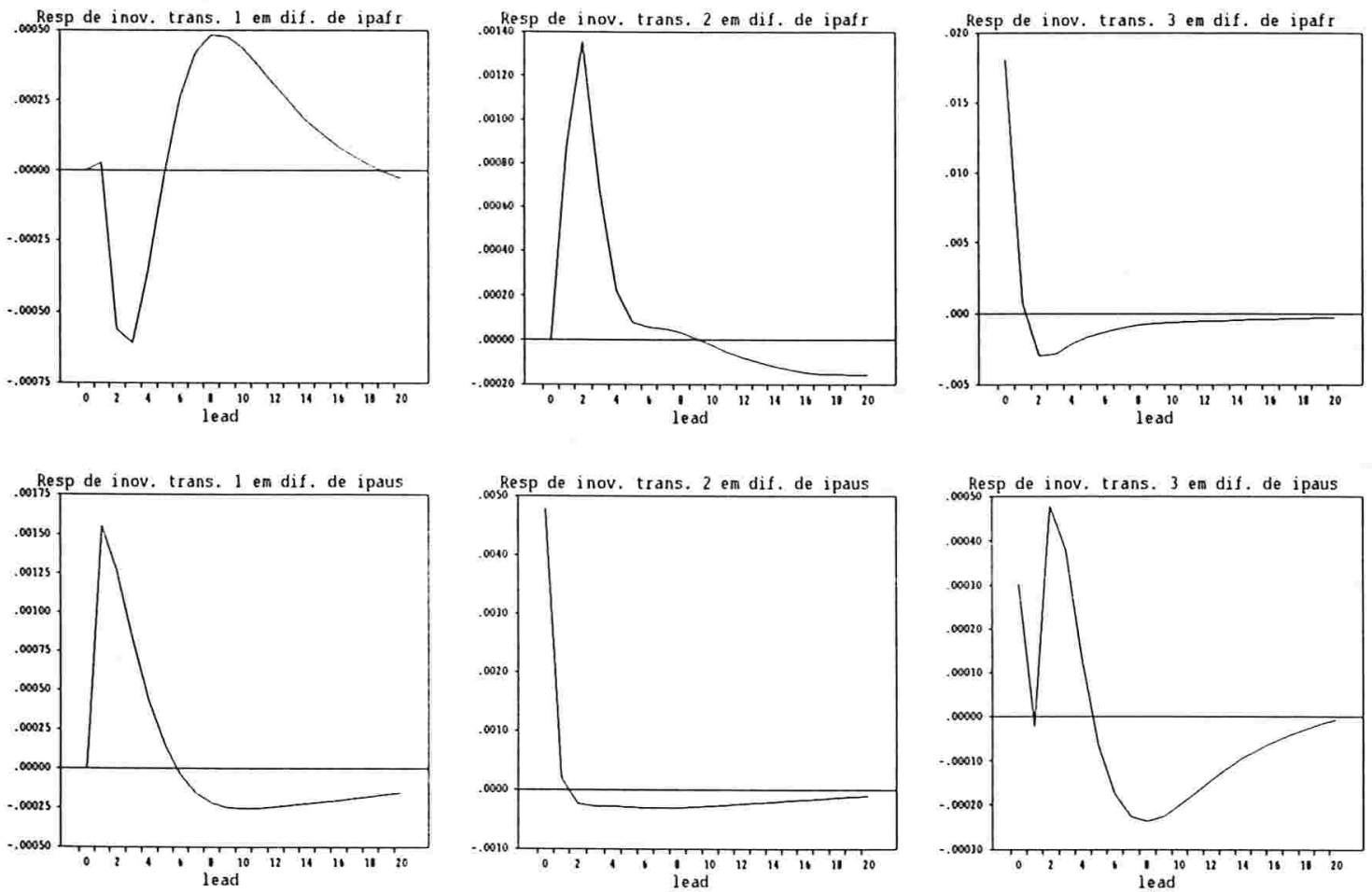


\section{Gráfico 7.8 (continuação)}

Respostas de Impulsos Estimados de $\Delta \chi_{t}$ para Inovações no Componente Transitório
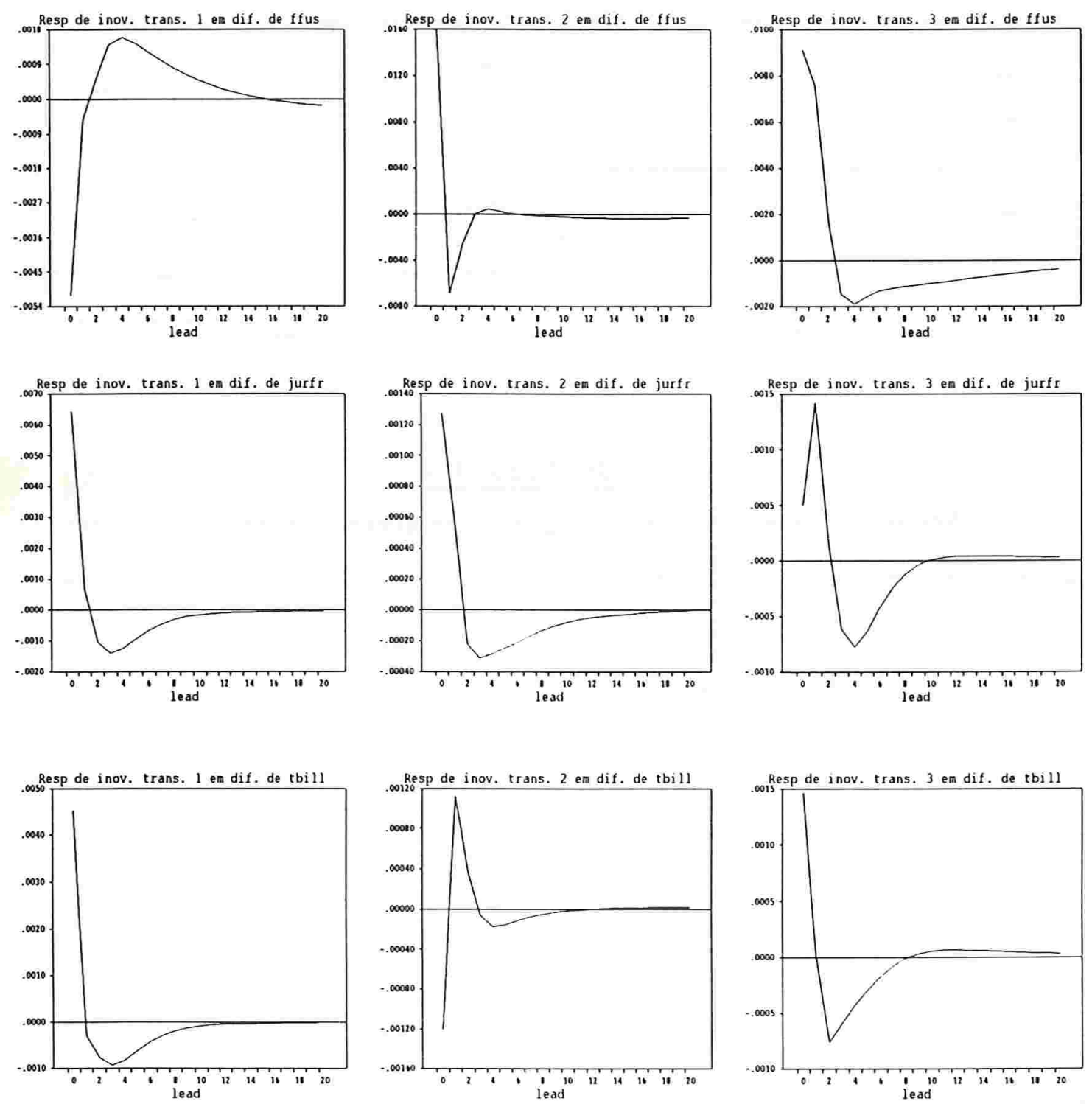


\section{Anexo Capítulo 7}

\section{Funções de Autocorrelação e Autocorrelação Parcial das 1 as Diferenças das séries do Modelo e das Relações de Co-integração}

\section{1) Diff(IPAFR)}

Dados trimestrais 95 períodos desde 1970:2 até 1993:4

$\begin{array}{lll}\text { Média } & 0,01 \quad \text { Desvio Padrão } & 0,03 \\ \text { Máximo } & 0,18 \mathrm{em} \mathrm{1974:1}\end{array}$

Maximo $\quad 0,18 \mathrm{em} \mathrm{1974:1}$

Mínimo $\quad-0,07$ em 1975:1

Correlograma( X ) e Correlograma Parcial( * )

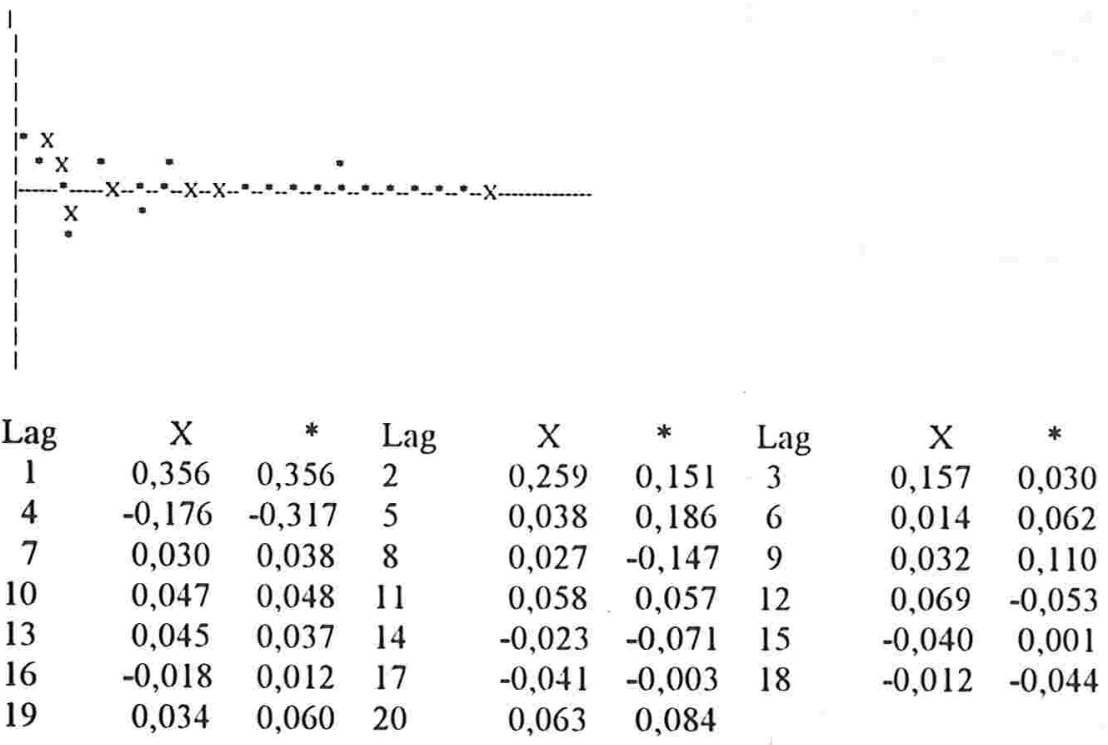

2) Diff(IPAUS)

Dados trimestrais 95 períodos desde 1970:2 até 1993:4
Média
0,01
Desvio Padrão
0,01
Máximo
0,05 em 1974:1
Mínimo $\quad-0,01$ em 1986:1 
Correlograma(X) e Correlograma Parcial( * )

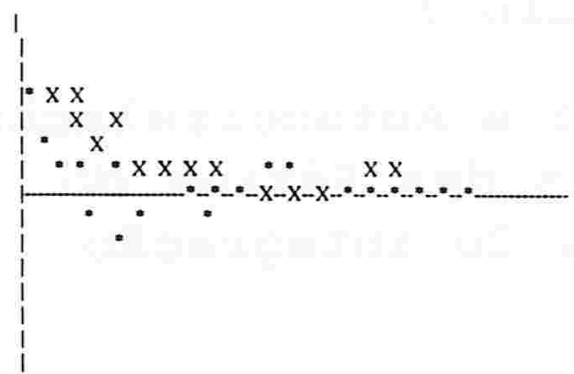

$\begin{array}{ccccccccc}\text { Lag } & \text { X } & * & \text { Lag } & \text { X } & * & \text { Lag } & \text { X } & * \\ 1 & 0,593 & 0,593 & 2 & 0,540 & 0,291 & 3 & 0,519 & 0,201 \\ 4 & 0,484 & 0,116 & 5 & 0,321 & -0,164 & 6 & 0,386 & 0,141 \\ 7 & 0,208 & -0,221 & 8 & 0,119 & -0,134 & 9 & 0,097 & 0,000 \\ 10 & 0,093 & 0,022 & 11 & 0,005 & 0,031 & 12 & -0,035 & -0,095 \\ 13 & 0,037 & 0,197 & 14 & 0,030 & 0,082 & 15 & -0,012 & -0,056 \\ 16 & 0,033 & 0,038 & 17 & 0,050 & -0,0319 & 18 & 0,009 & -0,011 \\ 19 & 0,090 & 0,045 & 20 & 0,134 & 0,057 & & & \end{array}$

\section{3) Diff(FFUS)}

Dados trimestrais 95 períodos desde 1970:2 até 1993:4

$\begin{array}{lll}\text { Média } & 0,00 \quad \text { Desvio Padrão } & 0,05\end{array}$

Máximo $\quad 0,12 \mathrm{em} \mathrm{1991:2}$

Mínimo $\quad-0,11$ em 1979:3

Correlograma( X ) e Correlograma Parcial( * )

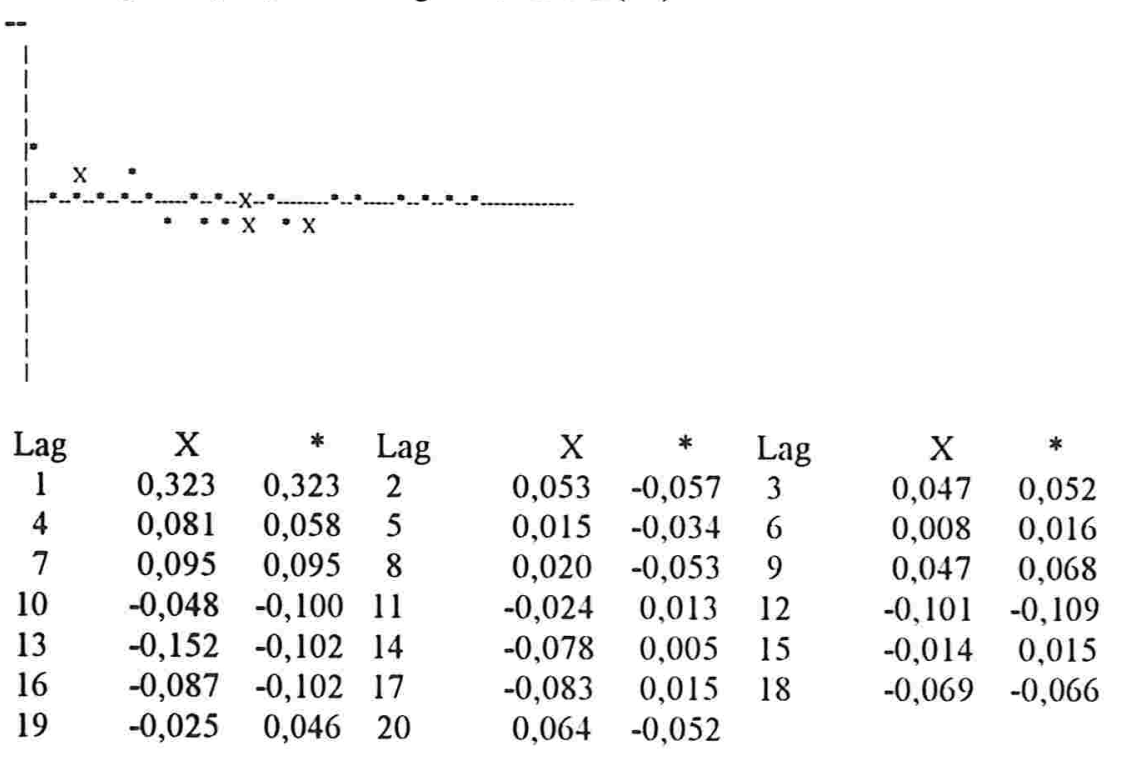


4) Diff(JURFR)

Dados trimestrais 95 períodos desde 1970:2 até 1993:4

Média $\quad-0,00 \quad$ Desvio Padrão 0,01

Máximo $\quad 0,04 \mathrm{em} \mathrm{1981:2}$

Mínimo $\quad-0,03$ em 1993:2

Correlograma( X ) e Correlograma Parcial( *)

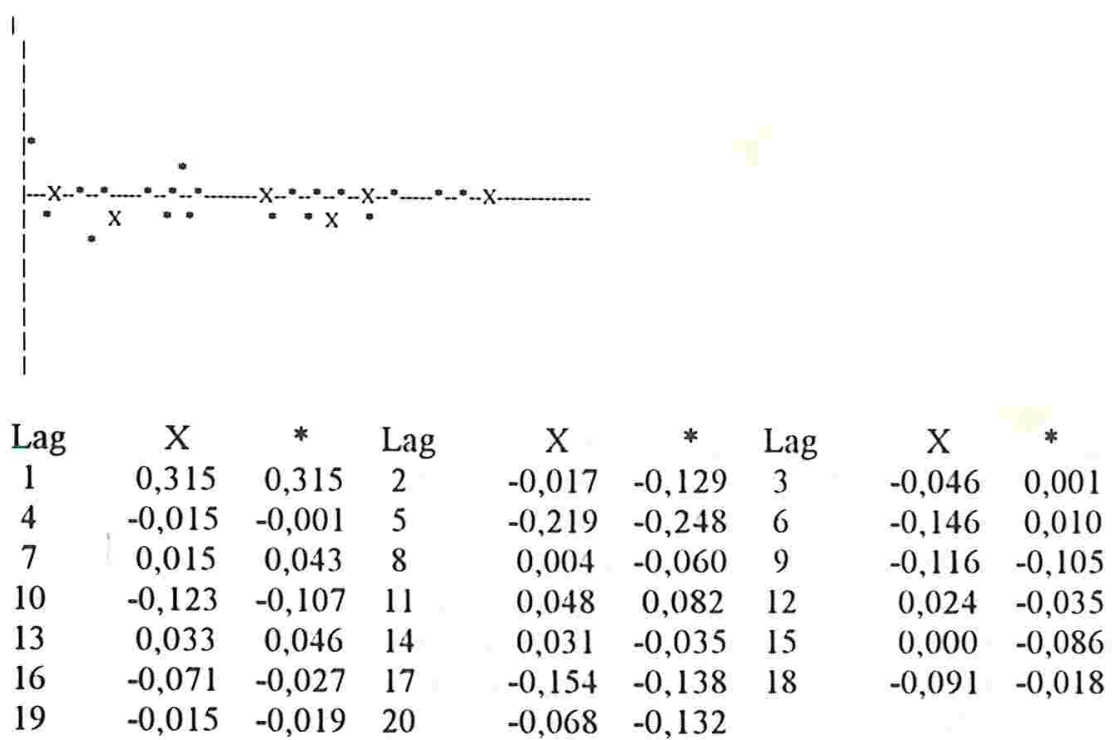

\section{5) Diff(TBILL)}

Dados trimestrais 95 períodos desde 1970:2 até 1993:4

Média $\quad-0,00 \quad$ Desvio Padrão 0,01

Máximo $\quad 0,06 \mathrm{em} \mathrm{1980:4}$

Mínimo $\quad-0,06 \mathrm{em}$ 1980:2

Correlograma( X ) e Correlograma Parcial( * )

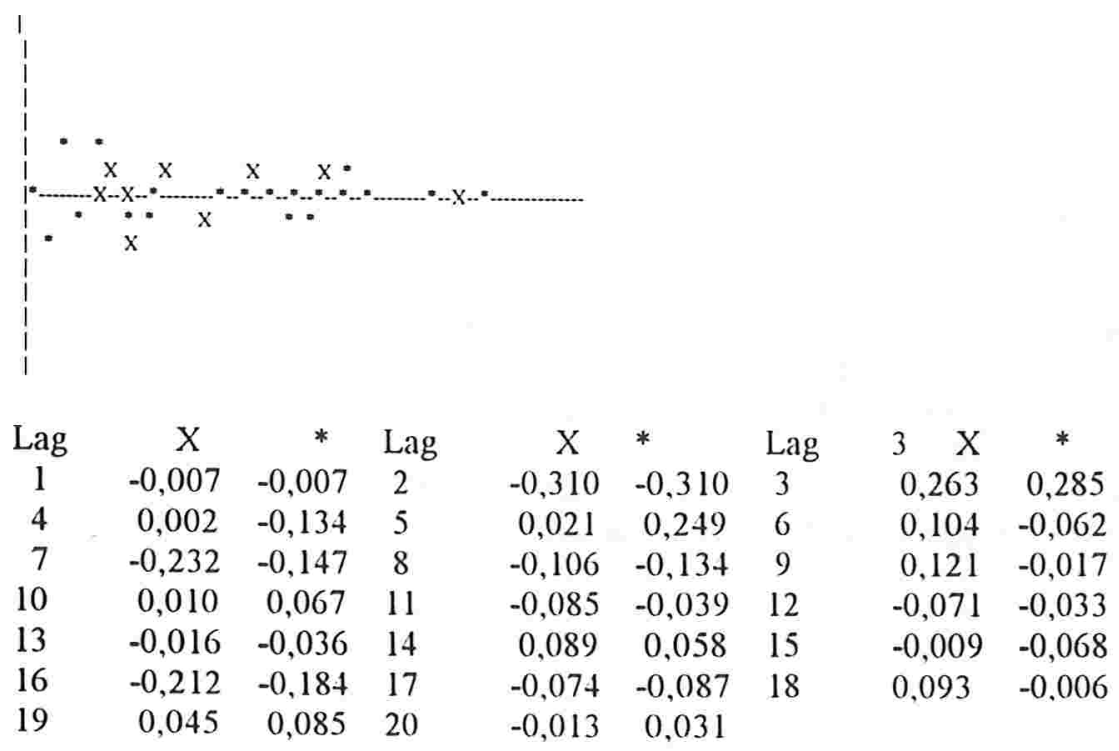


6) $1^{\mathrm{a}}$ Relação de Co-integração : ffus - ipafr + ipaus

Dados trimestrais 96 períodos desde 1970:1 até 1993:4
Média
1,91
Desvio Padrão
0,163

Máximo $\quad 2,35$ em 1985:1

Mínimo $\quad 1,62 \mathrm{em} \mathrm{1974:1}$

\section{Correlograma( X ) e Correlograma Parcial( * )}

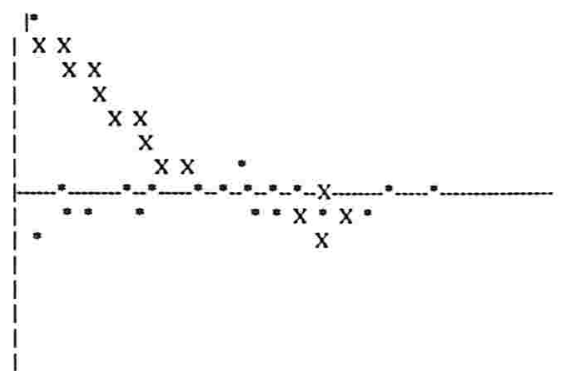

$\begin{array}{ccccccccc}\text { Lag } & \text { X } & * & \text { Lag } & X & * & \text { Lag } & X & * \\ 1 & 0,952 & 0,952 & 2 & 0,881 & -0,254 & 3 & 0,813 & 0,031 \\ 4 & 0,738 & -0,132 & 5 & 0,656 & -0,082 & 6 & 0,573 & -0,036 \\ 7 & 0,490 & -0,063 & 8 & 0,398 & -0,160 & 9 & 0,301 & -0,059 \\ 10 & 0,207 & -0,068 & 11 & 0,120 & 0,025 & 12 & 0,047 & 0,042 \\ 13 & -0,013 & 0,051 & 14 & -0,054 & 0,108 & 15 & -0,092 & -0,075 \\ 16 & -0,131 & -0,076 & 17 & -0,159 & 0,052 & 18 & -0,187 & -0,136 \\ 19 & -0,213 & -0,024 & 20 & -0,240 & -0,115 & & & \end{array}$

7) $2^{\text {a }}$ Relação de Co-integração : jurfr - tbill

Dados trimestrais 96 periodos desde 1970:1 até 1993:4

$\begin{array}{llll}\text { Média } & 0,02 & \text { Desvio Padrão } & 0,02\end{array}$

Máximo $\quad 0,08$ em 1993:1

Mínimo $\quad-0,05$ em 1980:4

Correlograma( X ) e Correlograma Parcial( * )

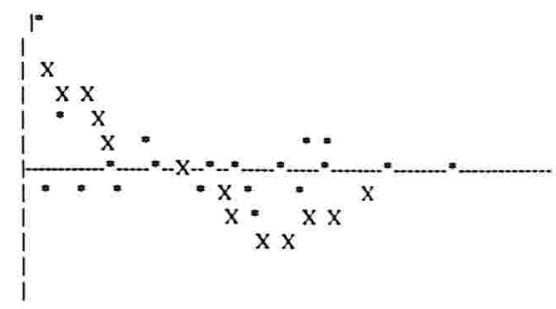

$\begin{array}{ccccccccc}\text { Lag } & \mathrm{X} & * & \mathrm{Lag} & \mathrm{X} & * & \mathrm{Lag} & \mathrm{X} & * \\ 1 & 0,798 & 0,798 & 2 & 0,571 & -0,178 & 3 & 0,483 & 0,250 \\ 4 & 0,375 & -0,194 & 5 & 0,230 & -0,050 & 6 & 0,099 & -0,126 \\ 7 & 0,013 & 0,006 & 8 & 0,005 & 0,128 & 9 & -0,001 & -0,032 \\ 10 & -0,049 & -0,055 & 11 & -0,110 & -0,127 & 12 & -0,143 & -0,037 \\ 13 & -0,181 & -0,110 & 14 & -0,220 & 0,007 & 15 & -0,309 & -0,236 \\ 16 & -0,413 & -0,124 & 17 & -0,416 & 0,058 & 18 & -0,335 & 0,113 \\ 19 & -0,262 & 0,102 & 20 & -0,207 & -0,006 & & & \end{array}$


As funções de autocorrelação das primeras diferenças das variáveis ipaus, ipafr, ffus, jurfr e tbill apresentam valores significativamente inferiores a 1, o que sugere que as 5 séries são integradas de ordem I(1).

Por outro lado, nas relações de co-integração, a fac da relação PPC sugere que nos encontramos num caso limíte de estacionariedade. Já na $2^{\text {a }}$ relação, a fac pode ser assimilada a uma série estacionária.

Observação : os valores $\pm 1,96 / \cdot n= \pm 0,20$ são considerados como consitituindo aproximadamente um intervalo de confiança para as funções de autocorrelação e autocorrelação parcial para uma sequência ruido branco. Para uma grande amostra, $95 \%$ das autocorrelações amostrais deveriam cair dentro destes limítes, se o processo em estudo é um ruido branco. Valores fora destes limites dão indicações sobre a ordem (p,q) dos processos ARMA observados pelas séries. Podemos já obervar que os valores das funções de autocorrelação parcial das primeras diferenças das séries ipafr, ipaus, ffus, jurfr e tbill não são significativamente diferentes de 0 a partir de determinados valores (entre 1 e 3), sugerindo que as séries seguem processos $A R(p)$. 


\section{Capítulo 8}

\section{Conclusões}

o objetivo deste trabalho foi a apresentação de uma técnica que permitisse a estimação da representação de Tendências Comuns de um vetor estocástico $\chi_{t}$, com $r$ vetores co-integrados, e da função de Respostas de Impulso no contexto desta representação.

Vimos que um vetor estocástico de tais características pode admitir as seguintes representações : 1) Modelo Autoregressivo Vetorial (VAR); 2) Mecanismo de Correção de Erro (MCE); 3) VAR Restrito; 4) Wold, e finalmente, 5) Tendências Comuns.

Foi mostrado como a partir da estimação da representação do MCE, os parâmetros das representações VAR e VAR Restrito, podem ser obtidos. O conhecimento dos parâmetros do VAR restrito permite o cálculo dos parâmetros da representação de Wold. Finalmente os parâmetros da representação de Tendências Comuns são identificados usando : 1) as relações que existem entre os parâmetros das representações de Wold e de Tendências, 2) as restrições impostas pela presença de co-integração e 3) restrições adicionais na matriz de variância-covariância do processo, e em alguns casos, outras restrições, que devemos impor para que o sistema seja exatamente identificado.

Usando as técnicas descritas, foi feita uma aplicação das mesmas para um vetor de dimensão 5 compreendendo as seguintes variáveis : preços e taxas de juros na França e nos EUA, e a taxa de câmbio FFr/US\$.

Com base em uma análise preliminar dos dados, foi admitido que o vetor era co-integrado de ordem 1,1, ou seja, seus componentes são I(1), mas existem combinações lineares dos mesmos que são I(0) i.e. estacionárias, que representam relações de longo prazo. Esta "análise preliminar" incluiu a análise gráfica e testes de Dickey Fuller de raizes unitárias, das séries em estudo e de séries associadas a determinadas relações (inspiradas pela teoria econômica) entre os componentes, supostamente estacionárias. Seguindo a linha de Johansen \& Juselius (1992) em seu estudo sobre a taxa de câmbio da Libra Esterlina com relação a uma cesta de moedas, foram consideradas particularmente : 1) a teoria da Paridade de Poder de Compra (PPC) e 2) a Paridade das Taxas de Juros não Coberta (PJNC). Básicamente, estas relações de 
longo prazo levavam a definição de dois vetores cointegrados : um compreendendo índices de preços e taxa de câmbio (em logaritmos) e outro contendo o diferencial das taxas de juros

Adicionalmente, foram consideradas outras possíveis relações de longo prazo, conhecidas nos mercados de capitais : 3) a taxa de câmbio FFr/US\$ guardando uma relação diretamente proporcional com o diferencial taxas de juros nos EUA-taxas de juros na França (o que é de certo modo contrário à PJNC) e 4) a taxa de câmbio FFr/US\$ guardando uma relação diretamente proporcional com o diferencial taxas de juros reais nos EUA-taxas de juros reais na França.

A estimação da representação de MCE do vetor em estudo foi feita usando o método de Johansen, que parte da função de verossimilhança da amostra. Os testes sobre o espaço de co-integração sugeriram a existência de 3 vetores cointegrados. Neste método, embora a matriz $\gamma \alpha^{\prime}$ ( $\gamma$ é a matriz dos pesos dados, para cada uma das variáveis, às relações de co-integração, e $\alpha$ é a matriz que determina as relações de co-integração) é exatamente identificada, $\alpha$ e $\gamma$ separadamente não são exatamente identificadas. O fato de que a dimensão de co-integração foi estimada em 3 tornou complexa a análise de dito espaço (qualquer vetor que é uma combinação linear das colunas de $\alpha$ pertence ao espaço).

Foram feitos uma série de testes considerando as relações discutidas acima. Estes testes sugeriram que definitivamente a relação $P P C$ pertence ao espaço de co-integração, e entra no sistema como variável explicativa. Com efeito, a hipótese nula de estacionariedade da relação $P P C$ não foi rejeitada, embora por escasa margem. O diferencial das taxas de juros, também pertence ao espaço de co-integração; no entanto quando $\gamma$ e $\alpha$ são consideradas conjuntamente, i.e. a matriz $\gamma \alpha^{\prime}$, os diferenciais das taxas de juros não entram nas equações do jeito que é de esperar segundo a PJNC, i.e. os sinais dos coeficientes não são os esperados. Faltava achar a base para o terceiro vetor co-integrado. Diferentes alternativas são possíveis. Uma é a relação 3 (ffustbill+jurfr); outra relação possível (que foi mais evidente com a análise gráfica dos componentes permanentes estimados das séries que foi feita a posteriori), é a relação das taxas de juros nos Estados Unidos com o tipo de câmbio.

Partindo das estimativas do MCE, foram estimados sucessivamente o VAR Restrito, a representação de Wold e a representação de Tendências Comuns. Como o número de vetores 
co-integrados estimado foi 3, então o número de tendências comuns estimado é dois. Na análise gráfica das tendências, vemos uma primeira tendência pronunciadamente crescente, sendo que a segunda, embora crescente, tem um comportamento mais errático.

A análise gráfica dos componentes permanentes das variáveis sugerem que preços nos EUA, e taxas de juros na França seguem predominantemente a $1^{a}$ tendência. A $2^{a}$ tendência tem forte influência sobre a taxa de câmbio FFr/US\$ e taxas de juros nos EUA, e, em menor medida sobre os preços na França.

Em outras palavras, a análise gráfica sugere a presência de uma tendência que parece estar em relação com o crescimento secular dos preços, embora ela influencie também as taxas de juros na França, e uma outra tendência mais associada ao mercado de câmbio e às taxas de juros nos EUA.

Foi feita a advertência de que a técnica utilizada para obter as tendências passa pelo cálculo de uma matriz que não é única. Por tanto, elas não são exatamente identificadas, de modo que a tentativa de dar um "nome" a estas tendências requer certos cuidados.

Foi calculada a função de Respostas de Impulso, no contexto da representação das Tendências Comuns, i.e. que permitiu analizar a reação do sistema para inovações nos componentes permanente e transitório daquela representação. Sem deixar de chamar a atenção de que a função de Respostas de Impulso não é única, foi observado que, para inovações no componente permanente, após 20 períodos, as variáveis preços por atacado na França e nos EUA não atingiram seu equilíbrio de longo prazo. As variáveis taxas de juros na França e nos EUA e taxa de câmbio FFr/US\$ chegam mais rapidamente ao equilíbrio, em torno de 10 trimestres. Em geral, para inovações no componente transitório, atinge-se o equilíbrio mais rápidamente. 


\section{Apêndice A}

\section{Algums Resultados de Algebra Matricial}

\section{A.1 Algumas definições e resultados em Algebra de Espaços Vetoriais}

Para mais detalhes sobre as definições e provas dos teoremas nesta seção, ver Graybill (1983, capítulos 1 e 5).

\section{A.1.1 Complemento Ortogonal de um Subespaço Vetorial}

Definição A.1 Subespaço de Vetores ortogonais em $E_{n}$ (Espaço Euclidiano). Sejam $S_{1}$ e $S_{2}$ dois subespaços em $E_{n}$. Se $\mathrm{x}_{1}{ }^{\prime} \mathrm{x}_{2}=0$ para cada vetor $\mathrm{x}_{1}$ em $\mathrm{S}_{1}$ e para cada vetor $\mathrm{x}_{2}$ em $\mathrm{S}_{2}$, então $S_{1}$ e $S_{2}$ são definidos como subespaços ortogonais em $E_{n}$, e denotamos isto por $\mathrm{S}_{1} \perp \mathrm{S}_{2}$.

Teorema A.1 Sejam $\left\{a_{1}, a_{2}, \ldots, a_{n}\right\}$ e $\left\{b_{1}, b_{2}, \ldots, b_{n}\right\}$ dois conjuntos de vetores em $E_{n}$ tais que $a_{i}{ }^{\prime} b_{j}=0$ para todo $i$ e $j$. O subespaço $\mathrm{S}_{1}$ gerado por $\left\{\mathrm{a}_{1}, \mathrm{a}_{2}, \ldots, \mathrm{a}_{\mathrm{n}}\right\}$ é ortogonal ao subespaço $\mathrm{S}_{2}$ gerado por $\left\{\mathrm{b}_{1}, \mathrm{~b}_{2}, \ldots, \mathrm{b}_{\mathrm{n}}\right\}$.

Definição A.2 Complemento Ortogonal de um Subespaço Vetorial em $E_{n}$. Seja $S_{1} u m$ subespaço vetorial em $E_{n}$. O subespaço vetorial $\mathrm{S}_{2}$ em $\mathrm{E}_{\mathrm{n}}$ é definido como complemento ortogonal de $S_{i}$ em $E_{n}$ se e somente se $S_{1} \perp S_{2}$ e $S_{1} \oplus S_{2}=E_{n}$ (a soma direta dos subespaços $S_{1}$ e $S_{2}$ é igual a $E_{n}$ ). Algumas vezes, o complemento ortogonal de $S_{1}$ é denotado por $S_{1}{ }^{\perp}$.

Teorema A.2 Para um determinado subespaço $S_{1}$ em $E_{n}$ o complemento ortogonal $\mathrm{S}_{1} \perp$ sempre existe e é único.

Teorema A.3 Seja $S_{1}$ um subespaço de $E_{n}$ e seja y qualquer vetor em $E_{n}$; então $y$ pode ser escrito como a soma de dois vetores $\mathrm{y}=\mathrm{x}_{1}+\mathrm{x}_{2}$, onde $\mathrm{x}_{1}$ está em $\mathrm{S}_{1}$ e $\mathrm{x}_{2}$ está no complemento ortogonal de $\mathrm{S}_{1}$. dados.

Corolario. $x_{1}$ e $x_{2}$ são vetores únicos para um $y$ e $S_{1}$ A.1.2 Espaço de Colunas e Nulidade de uma matriz

Definição A.3 Espaço de Colunas de uma Matriz. Seja A uma matriz $n \times m$. Denote as $m$ colunas de $A$ como vetores em $E_{n}$, de 
tal modo que $A=\left[a_{1}, \ldots, a_{m}\right]$. O espaço vetorial gerado por estas m colunas de A são definidas como o espaço de colunas de A. A dimensão deste espaço é igual ao número de colunas linearmente independentes de $A$, ou seja, ao posto de $A$.

Teorema A.4 Seja A uma matriz $n \times n$ não singular. O espaço de colunas de $A$ é $E_{n}$.

Definicão A.4 Nulidade de uma Matriz. Seja A uma matriz n $\times m$. A nulidade da matriz A é definida como o conjunto de vetores $\mathrm{S}$ onde :

$$
S=\left\{y: A y=0 ; \quad y \in E_{m}\right\}
$$

Teorema A.5 A nulidade de uma matriz A de dimensão $n \times m$ é um subespaço vetorial de $E_{n}$.

Teorema A.6 Seja A uma matriz de dimensão nxm. O espaço nulo de $A^{\prime}$ e o complemento ortogonal do espaço de colunas de A é o mesmo.

Teorema A.7 Seja A uma matriz de dimensão $n \times m$ e $B$ uma matriz de dimensão $m \times k$. O espaço das colunas de $A B$ é um subespaço do espaço das colunas de A.

Teorema A.8 Sejam A e B matrizes de dimensão $n \times m$. Existe uma matriz não singular $C$, de dimensão $m \times m$ tal que $A C=B$ se e somente se A e B tem o mesmo espaço de colunas.

Teorema A.9 Sejam A e B matrizes de dimensão $n \times m$. A condicão necessária e suficiente para que exista uma matriz $C$ de dimensão $m \times m$ tal que $A C=B$ é de que o espaço das colunas de B seja um subespaço do espaço das colunas de A.

Teorema A.10 Sejam A uma matriz de dimensão $m \times n$ de posto $r$ e $X$ uma matriz de dimensão $n \times p$, e o produto destas duas matrizes a matriz nula, ou seja :

$$
A X=0,
$$

então

(i) existe uma matriz $x$ de posto $n-r$ que satisfaz a equação acima,

(ii) o posto de $X$ não pode exceder $n-r$ 
Teorema A.11 Se A é uma matriz quadrada de dimensão $n$, com posto $r$, então existe uma matriz não nula $X$ tal que $A X=0$ see $r<n$.

Teorema A.12 Se A é uma matriz de dimensão $m \times n$ com $m<n$, então existe uma matriz não nula tal que $\mathrm{AX}=0$.

\section{A.2 Autovalores e Autovetores}

Os autovalores, ou valores característicos, ou raízes características de uma matriz quadrada $A$ de dimensão $m$ são as raízes do polinomio em $\lambda$ dado por $\operatorname{det}\left(A-\lambda I_{m}\right)$ ou $\operatorname{det}\left(\lambda I_{m}-A\right)$. $O$ determinante é chamado de determinante característico, e o polinômio de polinômio característico.

Um número $\lambda_{i}$ é um autovalor de $A$ se as colunas de $\left(A-\lambda_{i} I_{m}\right)$ são linearmente dependentes. Consequentemente, existe um vetor $v_{i}$, de dimensão $m \times 1$ tal que :

$$
\left(A-\lambda_{i} I_{m}\right) v_{i}=0 \quad \text { ou } A v_{i}=\lambda_{i} v_{i}
$$

Um vetor com esta propriedade é chamado de autovetor ou vetor característico correspondente a $\lambda_{i}$.

Regras :

(1) Se A é simétrica, então todos os autovalores são números reais.

(2) Os autovalores de uma matriz diagonal são seus elementos diagonais.

(3) Os autovalores de uma matriz triangular são seus elementos diagonais.

(4) Uma matriz de dimensão $m \times m$ tem no máximo $m$ autovalores.

(5) Sejam $\lambda_{1}, \ldots, \lambda_{m}$ os autovalores de uma matriz $A$ de dimensão $\mathrm{m} \times \mathrm{m}$, então $|\mathrm{A}|=\lambda_{1} \ldots \lambda_{\mathrm{m}}$.

(6) Sejam $\lambda_{i}$ e $\lambda_{j}$ distintos autovalores de $A$, com os correspondentes autovetores $v_{i}$ e $v_{j}$. Então $v_{i}$ e $v_{j}$ são linearmente independentes. 
(7) Todos os autovalores de uma matriz A de dimensão m×m tem módulo menor do que 1 see $\operatorname{det}\left(I_{m}-A z\right) \neq 0$ para $|z| \leq 1$, isto é, a polinomial det $\left(\mathrm{I}_{\mathrm{m}}-\mathrm{Az}\right)$ não tem raízes dentro e sobre 0 círculo unitário.

\section{A.3 Decomposição e Diagonalização de Matrizes.}

\section{A.3.1 A Forma Canônica Similar}

Derivação da Forma Canônica Similar. Seja A uma matriz de dimensão $\mathrm{m} \times \mathrm{m}$, com autovalores $\lambda_{1}, \ldots, \lambda_{\mathrm{m}}$ onde alguns autovalores podem ser repetidos. Cada autovalor $\lambda_{i}$ tem seu correspondente autovetor $u_{i}$ para o qual :

$$
\mathrm{Au}_{\mathrm{i}}=\lambda_{\mathrm{i}} \mathrm{u}_{\mathrm{i}} \text { para } i=1,2, \ldots, \mathrm{m}
$$

Isto é verdade para todas as raízes da equação característica, o que significa que para $\lambda_{k}$, de multiplicidade $m_{k}$, tem $m_{k}$ equações da forma apresentada em (A.1); se existem $m_{k}$ autovetores $p_{k}$ linearmente independentes correspondendo aos $\lambda_{k}$, teremos uma equação para cada vetor, enquanto que se não tem $m_{k}$ vetores $p_{k}$ linearmente independentes, podemos ainda ter $m_{k}$ equações com alguns $p_{k}$ sendo repetições de outros. Em todos os casos, o arranjo de equações apresentado em (A.1) pode ser escrito como :

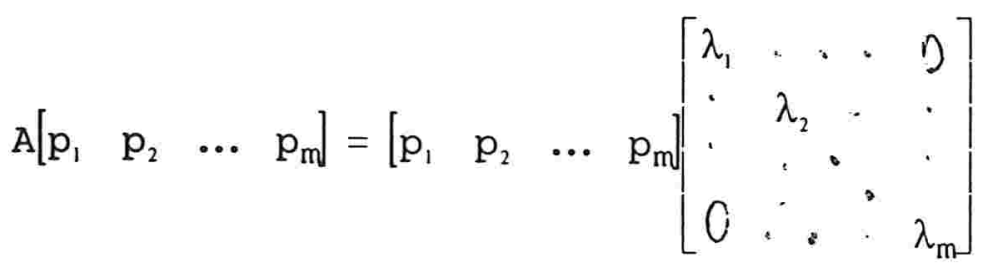

Definindo como $\mathrm{P}$ a matriz dos $\mathrm{n}$ autovetores, $e$ como $\Lambda$ a matriz diagonal dos autovalores, (A.2) pode ser escrito como :

$$
\mathrm{AP}=\mathrm{P} \Lambda
$$

$\Lambda$ é conhecida como a forma canônica similar. 
A forma (A.2), ou, de forma equivalente, a forma (A.2)', existe sempre. Nos casos em que $p_{i}$ se repete, é claro que $\mathrm{P}$ é uma matriz singular.

Nos casos em que $P$ é uma matriz não singular, ou seja, todos os autovalores são linearmente independentes, (A.2) pode ser reescrita como:

$$
\mathrm{P}^{-1} \mathrm{AP}=\left[\begin{array}{ccc}
\lambda_{1} & \cdots & 0 \\
\vdots & \ddots & \vdots \\
0 & \ddots & \lambda_{\mathrm{m}}
\end{array}\right]=: \Lambda \text { ou } \mathrm{A}=\mathrm{P} \Lambda \mathrm{P}^{-1}
$$

- produto no primeiro membro de (3) é conhecido como redução à forma canônica similar. A redução à forma canônica similar tem importantes implicações. Por exemplo, implica que, para A definida em (A.3) :

$$
\mathrm{A}^{j}=\left(\mathrm{P} \Lambda \mathrm{P}^{-1}\right)^{j}=\mathrm{P} \Lambda \mathrm{P}^{-1}
$$

Podem ser demonstradas as seguintes regras : Suponha que A é uma matriz real de dimensão $m \times m$, com autovalores $\lambda_{1}, \ldots, \lambda_{\mathrm{m}}$ que tem todos módulo menor que 1 , isto é, $\left|\lambda_{i}\right|<1$ para $i=1, \ldots, n$. Suponha também que $\Lambda$ e $P$ são as matrizes definidas em (A.3). Então :

(1) $\mathrm{A}^{j}=\mathrm{P} \Lambda \mathrm{P}^{-1} \longrightarrow \mathrm{j \rightarrow \infty} \longrightarrow 0$

(2) $\sum_{j=0}^{\infty} A^{j}=\left(I_{m}-A\right)^{-1}$ existe.

(3) A sequência $A_{j}, j=0,1,2, \ldots$, é absolutamente somável.

\section{A.3.2 Decomposição de matrizes simétricas}

Seja A uma matriz simétrica de dimensão m×m. Então existe uma matriz ortogonal $\mathrm{P}$ tal que : 


$$
\operatorname{PAP}=\Lambda=\left[\begin{array}{ccc}
\lambda_{1} & \ddots & 0 \\
\vdots & \ddots & \vdots \\
0 & \cdots & \lambda_{m}
\end{array}\right] \text { or } \mathrm{A}=\mathrm{P} \Lambda \mathrm{P}^{\prime}
$$

onde $\lambda_{i}$ são os autovalores de $A$ e as colunas de $P$ são os correspondentes autovetores. Aqui todas as matrizes são reais sendo que os autovalores de uma matriz simétrica são números reais. Denotando a i-ésima coluna de $P$ como $p_{i} e$ usando que $p^{\prime}{ }_{i} p_{j}=0$ para $i \neq j$, temos :

$$
\mathrm{A}=\mathrm{P} \Lambda \mathrm{P}^{\prime}=\sum_{i=1}^{\mathrm{m}} \lambda_{\mathrm{i}} \mathrm{p}_{\mathrm{i}} \mathrm{p}_{\mathrm{i}}^{\prime}
$$

Também, temos que :

$\mathrm{A}^{2}=\mathrm{P} \Lambda \mathrm{P} P \Lambda \mathrm{P}^{\prime}=\mathrm{P} \Lambda^{2} \mathrm{P}^{\prime}$

e mais geralmente :

$\mathrm{A}^{\mathrm{k}}=\mathrm{P} \Lambda^{\mathrm{k}} \mathrm{P}^{\prime}$

Se A é uma matriz positiva definida de dimensão $m \times m$, todos os autovalores de A são positivos, então a notação

$$
\Lambda^{1 / 2}:=\left[\begin{array}{ccc}
\sqrt{\lambda_{1}} & \cdots & 0 \\
\vdots & \cdots & \vdots \\
0 & \cdots & \sqrt{\lambda_{m}}
\end{array}\right]
$$

faz sentido. Definindo $\mathrm{Q}=\mathrm{P} \Lambda^{1 / 2} \mathrm{P}^{\prime}$, obtemos $\mathrm{QQ}=\mathrm{A}$. Em generalização da terminologia para números reais positivos, $Q$ pode ser chamada de raíz quadrada de $A$ e pode ser denotada como $A^{1 / 2}$.

\section{A.3.3 A Decomposição de Choleski de uma Matriz Positiva Definida}

Seja A uma matriz simétrica de dimensão m $\times m$. Então existe uma matriz triangular superior e uma matriz triangular inferior $\mathrm{P}$ com diagonal principal positiva tal que : 


$$
\mathrm{P}^{-1} \mathrm{AP}^{\prime-1}=\mathrm{I}_{\mathrm{m}} \quad \text { ou } \quad \mathrm{A}=\mathrm{PP}^{\prime}
$$

Similarmente, seja $A$ uma matriz positiva semi-definida como posto $(A)=n, m$, então existe uma matriz $P$ tal que :

$$
\mathrm{P}^{-1} \mathrm{AP}^{\prime-1}=\left[\begin{array}{cc}
\mathrm{I}_{\mathrm{n}} & 0 \\
0 & 0
\end{array}\right]
$$

Alternativamente, $\mathrm{A}=\mathrm{QQ}^{\prime}$, onde

$$
Q=P\left[\begin{array}{cc}
I_{n} & 0 \\
0 & 0
\end{array}\right]
$$

A decomposição $\mathrm{A}=\mathrm{PP}^{\prime}$, quando $\mathrm{P}$ é uma matriz triangular inferior com diagonal principal positiva, é às vezes chamada de decomposição de Choleski.

Para mais detalhes, ver Searle (1982), capítulo 11. 
Apêndice B

\section{Somas Infinitas de Matrices e Convergência Estocástica}

\section{B.1 Conceitos de Convergência Estocástica}

Seja $\chi_{1}, \chi_{2}, \ldots$ ou $\left\{\chi_{T}\right\}, T=1,2 \ldots$, uma sequência de variáveis aleatórias definidas em um espaço de probabilidade $(\Omega, M, P)$.

Definição B.1 A sequência $\left\{\chi_{\mathrm{T}}\right\}$ converge em probabilidade para uma variável aleatória $\chi$ (também definida em $(\Omega, M, P)$ ) se para cada $\varepsilon>0$,

$$
\begin{aligned}
& \lim _{T \rightarrow \infty} P\left(\left|\chi_{t}-\chi\right|>\varepsilon\right)=0, \quad \text { or equivalentemete } \\
& \lim _{T \rightarrow \infty} P\left(\left|\chi_{t}-\chi\right|<\varepsilon\right)=1
\end{aligned}
$$

Este tipo de convergência pode ser denotada por :

$$
\mathrm{p} \lim \chi_{\mathrm{T}}=\chi \quad \text { ou } \quad \chi_{\mathrm{T}} \stackrel{\mathrm{p}}{\longrightarrow} \chi
$$

Definição B.2 A sequência $\left\{\chi_{\mathrm{T}}\right\}$ converge quase certamente (q.c.) ou com probabilidade 1 para uma variável aleatória $\chi$ para cada $\varepsilon>0$,

$$
P\left(\lim _{\mathbb{I} \rightarrow \infty}\left|\chi_{t}-\chi\right|<\varepsilon\right)=1
$$

Este tipo de convergência pode ser denotada por :

$$
\chi_{\mathrm{T}} \stackrel{\mathrm{gc}}{\longrightarrow} \chi
$$

Definição B.3 A sequência $\left\{\chi_{\mathrm{T}}\right\}$ converge em média quadrática ou erro médio quadrático para uma variável aleatória $\chi$ se,

$$
\lim _{\mathrm{T} \rightarrow \infty} \mathrm{E}\left(\chi_{\mathrm{T}}-\chi\right)^{2}=0
$$


Este tipo de convergência pode ser denotada por :

$$
\chi_{\mathrm{T}} \stackrel{\mathrm{mq}}{\longrightarrow} \chi
$$

e requer que a média e a variança de $\chi$ e $\chi_{\mathrm{T}}$ existam.

\section{B.2 Somas Infinitas de Variáveis Aleatórias}

Definição B.4 Uma sequência infinita de números reais $\left\{a_{i}\right\}, i=0, \pm 1, \pm 2, \ldots$, é absolutamente somável se :

$$
\lim _{n \rightarrow \infty} \sum_{i=-n}^{n}\left|a_{j}\right|
$$

existe e é finito. O limite é usualmente denotado por :

$$
\sum_{i=-\infty}^{\infty}\left|a_{i}\right|
$$

A prova do seguinte teorema constitui a justificação para trabalhar com somas infinitas de variáveis aleatórias e a prova pode ser achada em Fuller (1976, págs.29-31).

Proposição B.1 (Existência de Somas Infinitas de Variáveis Aleatórias).

Suponha $\left\{a_{i}\right\}$ uma sequência de números reais absolutamente somável e $\left\{z_{t}\right\}, t=0, \pm 1, \pm 2, \ldots$, uma sequência de variáveis aleatórias satisfazendo :

$$
\mathrm{E}\left(\mathrm{z}_{\mathrm{t}}^{2}\right) \leq \mathrm{c}, \quad \mathrm{t}=0, \pm 1, \pm 2, \ldots,
$$

Então existe uma sequência de variáveis aleatórias $\left\{y_{t}\right\}$, $t=0, \pm 1, \pm 2, \ldots$, tal que :

$$
\sum_{i=-n}^{n} a_{i} z_{t-i} \longrightarrow y_{t} \quad \text { em média quadrática (B. 7) }
$$

e, em consequência : 


$$
p \lim \sum_{i=-n}^{n} a_{i} z_{t-i}=y_{t}
$$

Proposição B.2 (Momentos de Somas Infinitas de Variáveis Aleatórias).

Suponha que $\left\{z_{t}\right\}$ satisfaz as condições da proposição B.1, e que $\left\{a_{i}\right\}$ e $\left\{b_{i}\right\}$ são sequências absolutamente somáveis e

$$
y_{t}=\sum_{i=-\infty}^{\infty} a_{i} z_{t-i} \quad \text { e } \quad \chi_{t}=\sum_{-\infty}^{\infty} b_{i} z_{t-i}
$$

Então :

$$
\begin{aligned}
& E\left(y_{t}\right)=\lim _{n \rightarrow \infty} \sum_{i=-n}^{n} a_{i} E\left(z_{t-i}\right), \\
& E\left(y_{t} x_{t}\right)=\lim _{n \rightarrow \infty} \sum_{i=-n j=-n}^{n} \sum_{i}^{n} a_{i} b_{j}\left(z_{t-i} z_{t-j}\right)
\end{aligned}
$$

Em particular :

$$
E\left(y_{t}^{2}\right)=\lim _{n \rightarrow \infty} \sum_{i=-n j=-n}^{n} \sum_{i}^{n} a_{j}\left(z_{t-i} z_{t-j}\right)
$$

Prova : Fuller(1976, págs. 32-33).

Todos os conceitos anteriores podem ser extendidos a processos vetoriais.

Definição B.5 Uma sequência de matrizes de dimensão $n \times n$ $\left\{A_{i}=\left(a_{s t, i}\right)\right\}, i=0, \pm 1, \pm 2, \ldots$ é absolutamente somável se cada sequência $\left\{a_{s t, i}\right\} s, t=1, \ldots n$, é absolutamente somável.

Proposição B.3 (Existência de Somas Infinitas de Vetores Aleatórios)

Suponha $\left\{A_{i}\right\}$ uma sequência de matrizes n-dimensionais reais absolutamente somável e $\left\{z_{t}\right\}$ uma sequência de vetores aleatórios n-dimensionais satisfazendo :

$$
E\left(z_{t}^{\prime} z_{t}\right) \leq c, \quad t=0, \pm 1, \pm 2, \ldots,
$$


para alguma constante finita c. Então existe uma sequência de variáveis aleatórias n-dimensionais $\left\{y_{t}\right\}$ tais
que :

$$
\sum_{i=-n}^{n} A_{i} z_{t i} \longrightarrow y_{t} \quad \text { em média quadrática }
$$

Prova : Fuller (1976, págs. 29-31).

Proposição B.4 (Momentos de Somas Infinitas de Vetores Aleatórios).

Suponha que $\left\{z_{t}\right\}$ satisfaz as condições da proposição B.3, e que $\left\{A_{i}\right\}$ e $\left\{B_{i}\right\}$ são sequências de matrizes n-dimensionais absolutamente somáveis e

$$
y_{t}=\sum_{i=-\infty}^{\infty} A_{i} z_{t-i \prime} \quad \text { e } \quad \chi_{t}=\sum_{-\infty}^{\infty} B_{i} z_{t-i}
$$

Então :

$$
\begin{aligned}
& E\left(y_{t}\right)=\lim _{n \rightarrow \infty} \sum_{i=-n}^{n} A_{i} E\left(z_{t-i}\right), \\
& E\left(y_{t} x_{t}^{\prime}\right)=\lim _{n \rightarrow \infty} \sum_{i=-n j-n}^{n} \sum_{i}^{n} A_{i}\left(z_{t-i} z_{t-j}^{\prime}\right) B_{j}^{\prime}
\end{aligned}
$$

onde o limite de sequência de matrizes é a matriz dos limites das sequências dos elementos individuais.

Prova : Fuller (1976, págs. 32-33). 


\section{Apêndice C}

\section{Cálculo dos Coeficientes do Polinomio na Representação de $\mathrm{MCE}$}

Um processo $\left\{\boldsymbol{x}_{t}\right\}$, que segue um processo VAR(p), de acordo com as especificações que temos feito no capítulo 2:

$$
\mathrm{A}(\mathrm{L}) \chi_{t}=\rho+\rho^{*} \mathrm{D}_{t}+\varepsilon_{t}
$$

$\operatorname{com} A(L)=I_{n}-\sum_{j=1}^{p} A_{j} j^{j}$, pode ter a representação :

$$
A^{*}(L) \Delta \chi_{t}=\rho+\rho^{*} D_{t}-A(l) \chi_{t-1}+\varepsilon_{t}
$$

onde :

$$
A^{*}(L)=I_{n}-\sum_{i=1}^{p-1} A_{i}^{*} L^{i} \text { e } \quad A_{i}^{*}=-\sum_{j=i+1}^{p} A_{j}
$$

Demonstração.

(i) Escrevendo A(L) como:

$$
A(L)=A^{*}(L)(l-L)+A(l) L
$$

procuraremos fazer a correspondência dos termos dos polinômios $A(L)$ e $A^{*}(L)$, para obter os termos do polinômio $\mathrm{A}(\mathrm{L})^{\star}$ em função dos termos do polinômio $\mathrm{A}(\mathrm{L})$. Assim, desenvolvendo (C.4), obtemos :

$$
\begin{aligned}
& I_{n}-A_{1} L-A_{2} L^{2} \ldots-A_{p} D^{p}=\left(I_{n}-A_{1}^{*} L-A_{2}^{*} L^{2} \ldots-A_{p}^{*} I^{P}\right)(1-L)+\left(I_{n}-A_{1}-A_{2} \ldots-A_{p} L^{2}\right. \\
& =I_{n}-A_{1}^{*} L-A_{2}^{*} L^{2} \ldots-A_{p}^{*} I^{p}--I_{n} L-A_{1}^{*} L^{2}-A_{2}^{*} L^{3} \ldots-A_{p}^{*} I^{p 1}+I_{n} L-A_{1} L-A_{2} L \ldots-A_{p} L
\end{aligned}
$$

Reagrupando termos no lado direito de (C.5), segundo a potência, obtemos: 


$$
I_{n}-A_{1} L-A_{2} L^{2} \ldots-A_{p}{ }^{P}=I_{n}-\left(A_{1}^{*}+A_{1}+A_{2} \ldots+A p^{L}-\left(A_{2}^{*}-A_{1}^{*}\right)^{2}-\left(A_{3}^{*}-A_{2}^{*}\right) L^{3}-\ldots-\left(A_{p}^{*}-A_{p_{1}}^{*}\right) L^{P}+A_{1}^{*} p^{p}\right.
$$

Então, igualando as potências de L em (C.6), obtemos :

$$
\begin{aligned}
& A_{1}^{*}+A_{1}+\ldots+A_{p}=A_{1} \quad \Rightarrow \quad A_{1}^{*}=-\sum_{j=2}^{p} A_{j} \\
& A_{2}^{*}-A_{1}^{*}=A_{2} \Rightarrow \operatorname{por}(C .7) \Rightarrow A_{2}^{*}=-\sum_{j=3}^{p} A_{j}
\end{aligned}
$$

$\operatorname{Para} A_{i-1}^{*}, \quad A_{i-1}^{*}=-\sum_{j=1}^{p} A_{j}$,

então para $A_{i}^{*}$ :

$$
A_{i}^{*}-A_{i-1}^{*}=A_{i} \Rightarrow \operatorname{por}(C .8) \Rightarrow A_{i}^{*}=-\sum_{j=1}^{p} A_{j}
$$

Também, note que $A_{p}^{*}=0$, pois está multiplicando ${ }^{p+1}$, potência que não existe em $A(L)$.

(ii) Substituindo (C.4) em (C.1), obtemos (C.2), que era o que queriamos demonstrar 


\section{Apêndice D}

\section{o Método de Johansen para estimar Processos AR com Co-integração}

Para estimar os parâmetros em (2.53), ou seja, estimar $\gamma$, $\boldsymbol{\alpha}, \mathrm{A}_{1}{ }, \ldots \mathrm{A}_{\mathrm{p}-1}$, $\rho$ e $\rho^{*}$, o método de Soren Johansen pode ser usado (Johansen, 1988). Seja um AR(p) :

$$
\chi_{t}=A_{1} \chi_{t-1}+A_{2} x_{t-2}+\ldots+A_{p} x_{t-p}+\rho+\rho^{*} D_{t}+\varepsilon_{t}
$$

que pode assumir a seguinte representação :

$$
A^{*}(L) \Delta x_{t}=\rho+\rho^{*} D_{t}-A(1) x_{t-1}+\varepsilon_{t}
$$

onde $\left\{\boldsymbol{\varepsilon}_{\boldsymbol{t}}\right\}$ é uma sequência de variáveis aleatórias i.i.d. com distribuição de Gauss, de média zero e matriz de variância-covariância $\Lambda ; \quad \Delta:=1-\mathrm{L}$ é 0 operador de primera diferença. Também :

$$
A(1)=I_{n}-\sum_{j=1}^{p} A_{j}
$$

e $A^{*}(L)$ é dado por :

$$
A^{*}(L)=I_{n}-\sum_{i=1}^{p-1} A_{i}^{*} L^{i} \text { onde } A_{i}^{*}=-\sum_{j=1+1}^{p} A_{j}
$$

Definindo a matriz polinomial :

$$
|A(z)|=\left|A_{1} z+A_{2} z^{2}+\ldots+A_{p} z^{2}\right|
$$

vamos nos concentrar nos casos em que o polinomio tem raízes igual a 1 , assumindo que o resto das raízes estão fora do círculo unitário. Assim, a matriz A(1) é singular, ou seja, $|A(1)|=0$. Assumimos que o posto da matriz $A(1)$ é $r$, onde $r$ é $O$ número de vetores co-integrados, como foi explicado no capítulo 2. 
Sendo que a matriz $A(1)$ é singular, de posto $r$, ela pode ser escrita como $\mathrm{A}(1)=\gamma \boldsymbol{\alpha}^{\prime}$, para apropriadas matrices $\boldsymbol{\gamma}$ e $\boldsymbol{\alpha}$, de dimensão $n \times r$.

A função de verossimilhança para (D.1) está dada por :

$L\left(A_{1}^{*}, \ldots, A_{p-1}^{*}, \gamma, \alpha, \Lambda\right)=\prod_{t=1}^{T}\left[(2 \pi)^{-n / 2}|\Lambda|^{-1 / 2} \exp \left\{-\frac{1}{2}\left(\Delta \chi_{t}-\mu\right)^{\prime} \Lambda^{-1}\left(\Delta \chi_{t}-\mu\right)\right\}\right] \quad(D .4)$

ou

$\mathrm{L}\left(\mathrm{A}_{1}^{*}, \ldots, \mathrm{A}_{\mathrm{p}-1}^{*}, \gamma, \alpha, \Lambda\right)=(2 \pi)^{-\mathrm{T} / 2}|\Lambda|^{-T / 2} \exp \left\{-\frac{1}{2} \sum_{\mathrm{i}=1}^{T}\left(\Delta \chi_{t}-\mu\right)^{\prime} \Lambda^{-1}\left(\Delta \chi_{t}-\mu\right)\right\}$

onde :

$$
\mu=\mathrm{A}_{1}^{*} \Delta \chi_{t-1}-\ldots-\mathrm{A}_{\mathrm{p}-1}^{*} \Delta \chi_{\mathrm{t}-\mathrm{p}+1}-\gamma \alpha^{\prime} \chi_{\mathrm{t}-\mathrm{p}}-\rho-\rho^{*} \mathrm{D}_{\mathrm{t}}
$$

A maximização de (D.4) sobre os parâmetros $A_{1}^{*}, \ldots, A_{p-1}^{*}, \rho, \rho$ é simples, consistindo na aplicação de uma regressão de mínimos quadrados ordinários de $\Delta \chi_{t}+\gamma \alpha^{\prime} \chi_{t-1}$ nas diferenças desfasadas de $\chi_{t}$. Isto pode ser feito usando o seguinte procedimento : faça a regressão de $\Delta \chi_{t}$ sobre as diferenças desfasadas $\Delta \chi_{t-1}, \ldots, \Delta \chi_{t-p+1}$, o que fornece os resíduos $R_{0} t$, $e$ logo faça a regressão de $\chi_{t-1}$ sobre as diferenças

$\Delta \chi_{t-1}, \ldots, \Delta \chi_{t-p+1}$, o que fornece os resíduos $R_{1 t}$, onde $R_{i t}$, $i=0,1$, são matrices $n \times 1$. Uma vez efetuadas estas regressões, a função de verossimilhança concentrada fica proporcional a:

$$
L(\gamma, \alpha, \Lambda) \propto|\Lambda|^{-T / 2} \exp \left\{-\frac{1}{2} \sum_{i=1}^{T}\left(R_{0 t}+\gamma \alpha^{\prime} R_{1 t}{ }^{\prime} \Lambda^{-1}\left(R_{0 t}+\gamma \alpha^{\prime} R_{1 t}\right\}\right.\right.
$$

Para um $\alpha$ fixo, podemos maximizar (D.5) sobre $\gamma$ e $\Lambda$, fazendo uma regressão de Mínimos Quadrados Ordinários de $R_{\text {ot }}$ sobre $-\alpha^{\prime} R_{1 t}$, o que resulta em :

$$
\tilde{\gamma}(\alpha)=-S_{01} \alpha\left(\alpha^{\prime} S_{11} \alpha\right)^{-1}
$$

e 


$$
\tilde{\Lambda}(\alpha)=S_{00}-S_{01} \alpha\left(\alpha^{\prime} S_{11} \alpha\right)^{-1} \alpha^{\prime} S_{10}
$$

onde são definidas as matrices (de dimensAo $n \times n$ ) dos produtos dos momentos dos resíduos como

$$
S_{i j}=T^{-1} \sum_{t=1}^{T} R_{i \mathbb{I}} R_{j}^{\prime}, \text { onde } i, j=0,1
$$

Substituindo (D.6) e (D.7) en (D.5), o exponente de (D.5) se torna uma constante. Assim, a função de verossimilhança concentrada, en torno de $\alpha$, fica :

$$
\mathrm{L}(\alpha) \propto|\tilde{\Lambda}(\alpha)|^{-\frac{T}{2}}
$$

- que implica encontrar a solução do problema de minimização :

$$
\min \left|S_{00}-S_{01} \alpha\left(\alpha^{\prime} S_{11} \alpha\right)^{-1} \alpha^{\prime} S_{10}\right|
$$

onde a minimização é feita sobre todas as matrices $\alpha$ de dimensão $n \times r$.

Dada a conhecida relação de determinantes particionados (ver Graybill 1983, capítulo 8):

$$
\begin{aligned}
\left|\begin{array}{ll}
S_{00} & S_{01} \alpha \\
\alpha^{\prime} S_{10} & \alpha^{\prime} S_{11} \alpha
\end{array}\right| & =\left|S_{00} \| \alpha^{\prime} S_{11} \alpha-\alpha^{\prime} S_{10} S_{00}^{-1} S_{01} \alpha\right| \\
& =\left|\alpha^{\prime} S_{11} \alpha\right|\left|S_{00}-S_{01} \alpha\left(\alpha^{\prime} S_{11} \alpha\right)^{-1} \alpha^{\prime} S_{10}\right|
\end{aligned}
$$

temos que minimizar a seguinte relação, com relação a matriz $\alpha$ :

$$
\left|\alpha^{\prime} S_{11} \alpha-\alpha^{\prime} S_{10} S_{00}^{-1} S_{01} \alpha\right| /\left|\alpha^{\prime} S_{11} \alpha\right|
$$

Defina agora a matriz diagonal $D$ com os autovalores ordenados $\tilde{\lambda}_{1}>\ldots>\tilde{\lambda}_{\mathrm{n}}$ de $\mathrm{S}_{10} \mathrm{~S}_{00}{ }^{-1} \mathrm{~S}_{01}$ com relação a $\mathrm{S}_{11}$, ou seja, as soluções da equação:

$$
\left|\lambda S_{11}-S_{10} S_{00}^{-1} S_{01}\right|=0
$$


e a matriz $\mathrm{E}$ com os correspondentes autovetores. A seguinte expressão é então verdade :

$$
\mathrm{S}_{11} \mathrm{ED}=\mathrm{S}_{10} \mathrm{~S}_{00}^{-1} \mathrm{~S}_{01} \mathrm{E}
$$

onde $E$ é normalizado de tal maneira que :

$$
E^{\prime} S_{11} E=I
$$

Agora, escolha

$$
\alpha=\mathrm{E} \xi
$$

onde $\xi$ é uma matriz nxr. Substituindo $\alpha$ em (D.12), por $E$ $\xi$, e considerando a expressão (D.15), temos agora que
minimizar a seguinte expressão:

$$
\left|\xi^{\prime} \xi-\xi^{\prime} D_{\zeta}\right| /\left|\xi^{\prime} \xi\right|
$$

Isto pode ser feito com a escolha de $\xi$ como sendo os primeiros $r$ vetores unitários, ou escolhendo $\bar{\alpha}$ como sendo os primeiros $r$ autovetores de $S_{10} S_{00}^{-1} S_{01}$ com relação a $S_{11}$, ou seja, as primeiras $r$ colunas de $E$. Estas colunas são chamadas de variáveis canônicas, e os autovalores são as correlações canônicas ao quadrado de $\mathrm{R}_{1 \mathrm{t}}$ com relação a $\mathrm{R}_{0 t}$. Para mais detalhes destes cálculos, ver Anderson (1984, capítulo 12). Este tipo de análise é também chamada de regressão de posto reduzido.

Considerando a expressão (D.15), e substituindo $\tilde{\alpha}$ em (D.6) e (D.7), obtemos :

$$
\begin{aligned}
& \tilde{\gamma}=-S_{01} \tilde{\alpha}\left(\tilde{\alpha}^{\prime} S_{11} \tilde{\alpha}\right)^{-1}=-S_{01} \tilde{\alpha} \\
& \tilde{\Lambda}=S_{00}-S_{01} \tilde{\alpha} \tilde{\alpha}^{\prime} S_{10}=S_{00}-\tilde{\alpha} \tilde{\alpha}^{\prime}
\end{aligned}
$$

Finalmente a estimativa de $A(1)$ :

$$
\tilde{\mathrm{A}}(\mathrm{l})=\tilde{\gamma} \tilde{\alpha}^{\prime}=-\mathrm{S}_{01} \tilde{\alpha}\left(\tilde{\alpha}^{\prime} \mathrm{S}_{11} \tilde{\alpha}\right)^{-1} \tilde{\alpha}^{\prime}=-\mathrm{S}_{01} \tilde{\alpha} \tilde{\alpha}^{\prime}
$$

Por outro lado, por (D.9), (D.11) e (D.15), temos que a função de verossimilhança verifica a seguinte relação: 


$$
L^{-2 / T} \propto|\tilde{\Lambda}|=\left|S_{00}\right|\left|\tilde{\alpha}^{\prime} S_{11} \tilde{\alpha}-\tilde{\alpha}^{\prime} S_{10} S_{00}^{-1} S_{01} \tilde{\alpha}\right|
$$

ou seja,

$$
L^{-2 / T} \propto\left|\tilde{\alpha}^{\prime} S_{11} \tilde{\alpha}-\tilde{\alpha}^{\prime} S_{10} S_{00}^{-1} S_{01} \tilde{\alpha}\right|
$$

Por (D.15) e (D.16), (D.21) fica :

$$
L^{-2 / T} \propto\left|S_{00}\right| \mid I-\xi^{\prime} E^{\prime} S_{10} S_{00}^{-1} S_{01} \text { 走 } \mid
$$

Premultiplicando (D.14) por E', obtemos :

$$
\begin{gathered}
E^{\prime} S_{11} E D=E^{\prime} S_{10} S_{00}^{-1} S_{01} E \\
D=E^{\prime} S_{10} S_{00}^{-1} S_{01} E
\end{gathered}
$$

Substituindo (D.23) em (D.22), obtemos :

$$
L^{-2 / T} \propto\left|S_{00}\right|\left|I-\xi^{\prime} D_{\xi}^{\xi}\right|
$$

Assim, a maximização da verossimilhança, considerando que $D$ é a matriz dos autovalores ordenados $\tilde{\lambda}_{1}>\ldots>\bar{\lambda}_{n}$ de $S_{10} S_{00}{ }^{-1} S_{01}$ com relação a $S_{11}$, é dada por :

$$
\mathrm{L}_{\max }^{-2 / T}=\left|S_{00}\right| \prod_{i=1}^{r}\left(1-\tilde{\lambda}_{i}\right)
$$

Testando o posto de co-integração. Baseados na discussão acima, o método proposto nos permite estimar $A(1)$ e $\Lambda$ sem restrições com relação ao posto de $A(1)$, ou seja, com relação ao posto de $\alpha$. Podemos calcular os $n$ autovalores $e$ autovetores de uma vez, e logo realizar inferência com relação a quantas relações de co-integração são significativas, 0 que equivale a testar quantos dos autovalores $\lambda$ são iguais a 0 . testes:

Johansen \& Juselius tem desenvolvido dois tipos de

i) Teste da estatística do traço. Deseja-se testar:

$H_{0}: r=r_{0}, \quad H_{1}: r_{0}<r \leq n \quad r=0,1, \ldots$ 
Partindo de (D.25), construimos o ratio de verossimilhança da seguinte forma, para $r_{0}$ e um $r_{1}$, tal que
$r_{1}>r_{0}$.

$$
\begin{aligned}
-2 \ln Q\left(H\left(r_{0}\right) \mid H\left(r_{1}\right)\right) & =T\left[-\sum_{i=1}^{r_{1}} \ln \left(1-\lambda_{j}\right)+\sum_{i=1}^{r_{0}} \ln \left(1-\lambda_{j}\right)\right] \\
& =-\mathrm{T} \sum_{i=1}^{\zeta_{1}+1} \ln \left(1-\lambda_{j}\right)
\end{aligned}
$$

Por exemplo, si temos :

$\mathrm{H}_{0}: \mathrm{r}=0, \mathrm{H}_{1}=\mathrm{r}=2$, o teste será :

$$
=-T \sum_{i=1}^{2} \ln \left(1-\lambda_{i}\right)
$$

ii) Teste da estatística do $\lambda_{\max }$. Considere os $\mathrm{n}$ valores estimados de $\lambda$, sequêncialmente de maior a menor, e teste em cada sequência si o i-ésimo autovalor é significativamente diferente de 0 . Si o autovalor $\lambda_{S}$ não é significativamente diferente de 0 , para um certo grau de significância, então podemos inferir que o posto de $\alpha$ é $n-s$, e o teste acaba aí.

Em outras palavras, o teste é baseado na comparação :

$$
\mathrm{H}_{0}=(r-1), \mathrm{H}_{1}=(r) \text {, }
$$

$$
\lambda_{\max }=-T \ln \left(1-\tilde{\lambda}_{1}\right)
$$

As distribuições asintóticas das estatísticas em (D.26) e (D.27) não são padrão. Em particular não são distribuições $\chi^{2}$, sendo versões multivariadas da distribuição de Dickeypor certas distribuições são convenientemente descritas Brownianos integrais estocásticas, envolvendo processos simulação. As podem ser tabuladas por diferença n-r. Por dependen simplesmente da porções selecionadas tanto, é possivel computar algumas Johansen \& Juselius (1990). 


\title{
Apêndice E
}

\author{
Dados
}

\section{INDICE DE PREÇOS POR ATACADO FRANÇA}

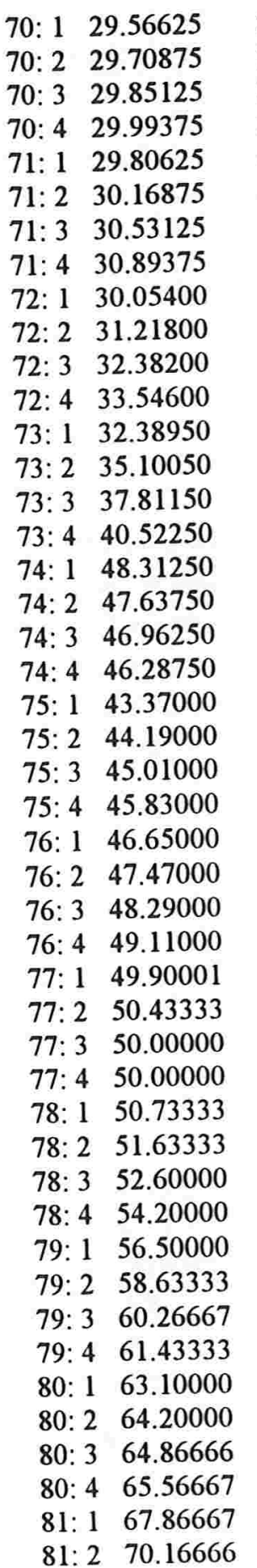

\begin{tabular}{|c|c|c|c|}
\hline 39.13334 & 5.547400 & 9.826667 & 7.249250 \\
\hline 39.10000 & 5.524467 & 9.093333 & 6.721750 \\
\hline 39.33334 & 5.519633 & 8.293334 & 6.194250 \\
\hline 39.63333 & 5.521333 & 7.533333 & 5.666750 \\
\hline 40.16667 & 5.517233 & 6.096667 & 4.451875 \\
\hline 40.46667 & 5.522900 & 6.616666 & 4.382625 \\
\hline 40.63334 & 5.516066 & 5.763333 & 4.313375 \\
\hline 40.76667 & 5.491000 & 5.583333 & 4.244125 \\
\hline 41.33333 & 5.100667 & 5.110000 & 2.957250 \\
\hline 41.50000 & 5.022000 & 4.403334 & 3.699750 \\
\hline 42.03333 & 5.004333 & 3.813334 & 4.442250 \\
\hline 42.23333 & 5.050000 & 6.263333 & 5.184750 \\
\hline 43.73333 & 4.793334 & 7.486666 & 6.724125 \\
\hline 45.03334 & 4.429333 & 7.613333 & 6.935375 \\
\hline 46.43333 & 4.186666 & 9.270000 & 7.146625 \\
\hline 47.13334 & 4.406667 & 11.28000 & 7.357875 \\
\hline 49.70000 & 4.935333 & 13.05333 & 8.654000 \\
\hline 51.03334 & 4.875333 & 12.77000 & 8.142000 \\
\hline 53.56667 & 4.784000 & 13.67333 & 7.630000 \\
\hline 55.93333 & 4.645100 & 12.48333 & 7.118000 \\
\hline 56.73333 & 4.282567 & 10.18333 & 6.606000 \\
\hline 57.43333 & 4.081533 & 7.733333 & 6.094000 \\
\hline 58.83333 & 4.357234 & 7.160000 & 5.582000 \\
\hline 59.93333 & 4.424266 & 6.623333 & 5.070000 \\
\hline 59.96666 & 4.522734 & 7.063333 & 4.853333 \\
\hline 60.43333 & 4.702600 & 7.570000 & 5.286666 \\
\hline 60.80000 & 4.907400 & 8.986667 & 5.116667 \\
\hline 61.90000 & 4.985800 & 10.62000 & 4.566667 \\
\hline 63.13334 & 4.976533 & 9.833333 & 4.680000 \\
\hline 64.46667 & 4.952500 & 9.070001 & 4.826667 \\
\hline 65.10000 & 4.889133 & 8.496667 & 5.570000 \\
\hline 66.23333 & 4.845500 & 8.876667 & 6.160000 \\
\hline 67.50000 & 4.756433 & 9.673333 & 6.746667 \\
\hline 69.26667 & 4.605300 & 8.106667 & 7.356667 \\
\hline 70.43334 & 4.387234 & 7.293334 & 8.180000 \\
\hline 72.06667 & 4.302033 & 6.850000 & 10.34000 \\
\hline 74.46667 & 4.268000 & 6.696667 & 10.26667 \\
\hline 76.33334 & 4.707600 & 7.343334 & 9.633333 \\
\hline 78.23333 & 4.234334 & 10.26000 & 10.80667 \\
\hline 81.23333 & 4.141200 & 11.86333 & 13.67333 \\
\hline 84.56667 & 4.149134 & 12.37667 & 15.26333 \\
\hline 86.76666 & 4.212101 & 12.48333 & 8.590000 \\
\hline 89.33334 & 4.121400 & 11.57667 & 10.18333 \\
\hline 91.33334 & 4.421533 & 10.94667 & 16.75667 \\
\hline 93.86667 & 4.859767 & 11.12333 & 14.84667 \\
\hline 96.06666 & 5.418533 & 16.06333 & 16.17333 \\
\hline
\end{tabular}

TAXA TAXAS DE JUROS CÂMBIO CURTO PRAZO (3 MESES) FFR/US\$ FRANÇA EUA 


\begin{tabular}{|c|c|c|c|c|c|}
\hline $81: 3$ & 00 & 96.76666 & 5.808400 & 17.92333 & 16.97333 \\
\hline $81: 4$ & 75.70000 & 97.96665 & 5.651633 & 16.09000 & 13.27333 \\
\hline $82: 1$ & 78.73333 & 98.93333 & 5.994867 & 15.17667 & 14.58000 \\
\hline $82: 2$ & 80.43333 & 99.20000 & 6.279867 & 16.40000 & 14.51667 \\
\hline $82: 3$ & 80.80000 & 100.4000 & 6.941767 & 14.58000 & 10.56000 \\
\hline $82: 4$ & 81.93333 & 101.4667 & 7.073100 & 13.32333 & 8.590000 \\
\hline 83: 1 & 84.36666 & 101.1000 & 6.887367 & 12.77333 & 8.430000 \\
\hline $83: 2$ & 86.43333 & 101.2667 & 7.469967 & 12.54000 & 8.256667 \\
\hline $83: 3$ & 88.40000 & 101.8000 & 7.960133 & 12.50333 & 9.163334 \\
\hline $83: 4$ & 90.40000 & 102.3333 & 8.167733 & 12.30000 & 8.889999 \\
\hline $84: 1$ & 92.73334 & 103.5333 & 8.305667 & 12.41333 & 9.433333 \\
\hline $84: 2$ & 95.00000 & 103.7333 & 8.331699 & 12.30333 & 10.76000 \\
\hline $84: 3$ & 96.50000 & 103.7333 & 8.960234 & 11.41333 & 11.25667 \\
\hline $84: 4$ & 97.50000 & 104.0333 & 9.362733 & 10.68000 & 8.923333 \\
\hline $85: 1$ & 98.83334 & 104.1333 & 9.960134 & 10.60667 & 8.346667 \\
\hline $85: 2$ & 100.2667 & 104.6667 & 9.407101 & 10.28667 & 7.633334 \\
\hline $85: 3$ & 100.9000 & 104.3333 & 8.686833 & 9.803333 & 7.876667 \\
\hline $85: 4$ & 100.0000 & 105.5000 & 7.886833 & 9.076667 & 7.763334 \\
\hline $86: 1$ & 99.00000 & 104.0333 & 7.207667 & 8.693334 & 7.513334 \\
\hline $86: 2$ & 97.83334 & 102.7667 & 7.146767 & 7.363333 & 6.576666 \\
\hline $86: 3$ & 96.83334 & 102.5333 & 6.777700 & 7. 106667 & 5.813334 \\
\hline $86: 4$ & 96.10000 & 103.5667 & 6.572234 & 7.633333 & 5.590000 \\
\hline $87: 1$ & 96.40000 & 104.1333 & 6.127767 & 8.883334 & 6.356667 \\
\hline $87: 2$ & 97.10000 & 105.3333 & 6.025366 & 8.256667 & 7.093333 \\
\hline $87: 3$ & 97.70000 & 105.8667 & 6.135233 & 8.140000 & 7.166667 \\
\hline $87: 4$ & 98.53333 & 106.1000 & 5.754467 & 8.993333 & 7.873333 \\
\hline 88: 1 & 99.53333 & 106.2333 & 5.668800 & 8.166667 & 6.906667 \\
\hline 88: 2 & 100.5333 & 107.4000 & 5.778667 & 7.840000 & 7.473333 \\
\hline 8: 3 & 102.7000 & 108.6333 & 6.318900 & 7.743333 & 8.373333 \\
\hline$: 4$ & 105.2000 & 109.7333 & 6.061400 & 8.286667 & 9.043334 \\
\hline$: 1$ & 108.8000 & 111.6333 & 6.294100 & 8.956667 & 9.746667 \\
\hline$: 2$ & 108.4133 & 113.8333 & 6.540733 & 8.770000 & 9.713333 \\
\hline$: 3$ & 108.4000 & 113.7000 & 6.454567 & 9.113334 & 8.913333 \\
\hline$: 4$ & 107.3000 & 115.0667 & 6.043000 & 10.37000 & 8.540000 \\
\hline$: 1$ & 106.8333 & 117.4000 & 5.721134 & 10.91667 & 8.336667 \\
\hline $0: 2$ & 106.9700 & 117.5667 & 5.643200 & 9.906666 & 8.410000 \\
\hline $0: 3$ & 105.7000 & 119.3000 & 5.343367 & 10.15333 & 8.110001 \\
\hline $0: 4$ & 108.0333 & 122.4000 & 5.061633 & 10.02333 & 8.053333 \\
\hline $1: 1$ & 106.3000 & 121.5333 & 5.204233 & 9.816667 & 6.846667 \\
\hline 1: 2 & 105.2400 & 121.6000 & 5.873167 & 9.390000 & 6.106667 \\
\hline $1 \cdot 3$ & 104.0000 & 121.5667 & 5.928767 & 9.453333 & 5.776667 \\
\hline 1: 4 & 103.8567 & 122.1333 & 5.554333 & 9.526668 & 4.993333 \\
\hline$: 1$ & 103.7433 & 122.0333 & 5.479700 & 9.970000 & 4.226667 \\
\hline $2: 2$ & 104.0767 & 123.1667 & 5.440600 & 9.963334 & 3.986667 \\
\hline $2: 3$ & 103.0600 & 123.5333 & 4.959967 & 10.51000 & 3.363333 \\
\hline $2: 4$ & 102.2967 & 124.0667 & 5.259500 & 10.87667 & 3.596667 \\
\hline 3: 1 & 101.3767 & 124.4667 & 5.543566 & 11.69333 & 3.213333 \\
\hline $3: 2$ & 100.6367 & 125.6000 & 5.455733 & 7.993333 & 3.226667 \\
\hline 3: 3 & 99.55000 & 124.5000 & 5.818433 & 7.540000 & 3.200000 \\
\hline$: 4$ & 98.92000 & 124.4000 & 5.833467 & 6.706666 & 3.343333 \\
\hline
\end{tabular}




\section{Referências Bibliográficas}

Ardeni, P.G. \& D. Lubian (1989), "Is There Trend Reversion in Purchasing Power Parity?", European Economic Review, 35, 1035-1055.

Campbell, J.Y., and R.J. Shiller (1988), "Interpreting Cointegrated Models", Journal of Economic Dynamic and Control, 12, 505-522.

Davidson, R. e Mackinnon, J. G, Estimation and Inference in Econometrics, New York : Oxford University Press, 1993.

Edison, H. J., Joseph E. Gagnon \& William R. Melick (1994), "Understanding the Empirical Literature on Purchasing Power Parity: The Post-Bretton Woods Era", Board of Governors of the Federal Reserve System, Division of International Finance, Washington D.C.: Unpublished.

Edison, H. J. \& Klovland, Jan T. (1987), "A Quantitative Reassessment of the Purchasing Power Parity Hypothesis : Some Evidence on Norway and the United States", Journal of Applied Econometrics, 2, 309-334.

Engle, R. F. \& C. W. J. Granger (1987), "Cointegration
and Error Correction: Representation, Estimation Testing", Econometrica, 55, 251-276.

Ericsson, N. R. (1992), "Cointegration, Exogeneity, and Policy Analysis: An Overview", Journal of Policy Modelling, $14(3), 251-280$.

Frankel, O.J.A. (1979), "On the Mark: A Theory of Floating Exchange Rates based on Real Interest Differentials", American Economic Review 69, 610-622.

Fuller, W. A. (1976), Introduction to Statistical Time Series, New York : John Wiley.

Gourieroux, C. \& A. Monfort (1983), Cours de Séries Temporelles, Paris : Economica. 
Graybill, F. A. (1983), Matrices with Applications in Statistics, Watdsworth International Group, Belmont,
California, 2a edição.

Harvey, A. C. (1990), The Econometric Analysis of Time Series, London : Philip Allan.

Johansen, S .

(1988), "Statistical Cointegration Vectors", Journal of Economics Dynamics and
Control, 12, 231-254. Cointegration Vectors", Journal of Economics Dynamics and
Control, 12, 231-254. Control, 12, 231-254.

Johansen, S. \& K. Juselius (1990), "Maximun Likelihood Estimation and Inference on Cointegration With Applications to the Demand for Money", Oxford Bulletin of Economics and
Statistics, 52, 169-210.

Johansen, S. (1991), "The Power Function for the Likelihood Ratio Test for Cointegration", em: J. Gruber, ed., Econometric Decision Models: New Methods of Modelling and Applications (Springer Verlag, New York, NY) 336-357.

Johansen, S. \& K. Juselius (1992), "Testing structural Hypotheses in a Multivariate Cointegration Analysis of the PPP and the UIP for UK", Journal of Econometrics, 53, 211-

Kim, Y. (1990), "Purchasing Power Parity in the Long-Run: A Cointegration Approach", Journal of Money, Credit and Banking, 22, 491-503.

King, R. G., C. I. Plosser, J. H. Stock, \& M. W. Watson (1991), "Stochastic Trends and Economic Fluctuations", NBER
Working Paper No. 2229 .

King, R. G., C. I. Plosser, J. H. Stock, \& M. W. Watson (1991), "Stochastic Trends and Economic Fluctuations",
American Economic Review, 81, 819-840.

Lutkepohl, H., Introduction to Multiple Time Series Analysis, Berlin : Springer-Verlag.

Lutkepohl, H. \& H.E. Reimers (1992), "Impulse Response Analysis of Co-integrated systems", Journal of Economics Dynamics and Control, 16, 53-78.

Mellander E., A. Vredin e A. Warne (1992), "Stochastic Trends and Economic Fluctuations in a Small Economy", Journal of Applied Econometrics, Vol. 7, 369-394. 
Nelson C. R. \& C. I. Plosser (1982), "Trends and Random Walks in Macroeconomic Time Series", Journal of Monetary Economics, 10, 139-162.

Searle Shayle R. (1982), Matrix Algebra Useful for Statistics, John Wiley \& Sons.

Stock, J. H. \& M. W. Watson (1988), "Testing for Common Trends", Journal of the American Statistical Association, 83, 1097-1107.

Valls Pereira, P.L (1991), "Cointegração e suas Representações: uma Resenha", Revista de Econometria, 11 , no 2, 185-216.

Warne, A. "A Common Trends Model : Identification, Estimation and Asymptotics", Manuscrito Não Publicado, Stockholm School of Economics, Suecia. 